
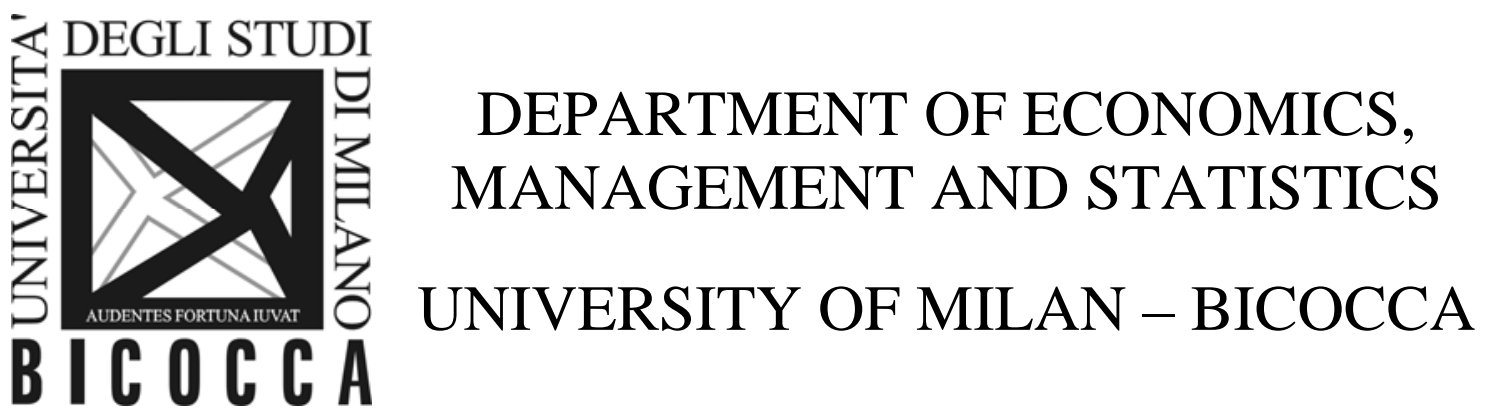

DEMS WORKING PAPER SERIES

Factor Vector Autoregressive Estimation of Heteroskedastic Persistent and Non Persistent Processes Subject to Structural Breaks: New Insights on the US OIS Spreads Term Structure

Claudio Morana

No. 233 - March 2013

Dipartimento di Economia, Metodi Quantitativi e Strategie di Impresa Università degli Studi di Milano - Bicocca http://dems.unimib.it/ 


\title{
Factor Vector Autoregressive Estimation of Heteroskedastic Persistent and Non Persistent Processes Subject to Structural Breaks: New Insights on the US OIS Spreads Term Structure
}

\author{
Claudio Morana \\ Dipartimento di Scienze Economiche, Metodi Quantitativi e Strategie d'Impresa \\ Università di Milano - Bicocca \\ CeRP-Collegio Carlo Alberto (Moncalieri, Italy) \\ Fondazione ENI Enrico Mattei (FEEM, Milano) \\ International Centre for Economic Research (ICER, Torino)
}

First draft December 2010; this draft March 2013

\begin{abstract}
In the paper a general framework for large scale modeling of macroeconomic and financial time series is introduced. The proposed approach is characterized by simplicity of implementation, performing
\end{abstract}

*Address for correspondence: Claudio Morana, Università di Milano - Bicocca, Facoltà di Economia, Dipartimento di Economia Politica, Piazza dell'Ateneo Nuovo 1, 20126, Milano, Italy. E-mail: claudio.morana@unimib.it.

${ }^{\dagger}$ A previous version of the paper was presented at the Nineteenth Annual Symposium of the Society for Non Linear Dynamics and Econometrics, the Fourth Annual Conference of the Society for Financial Econometrics, the 65th European Meeting of the Econometric Society (ESEM), the 2011 NBER-NSF Time Series Conference, and the 5th CSDA International Conference on Computational and Financial Econometrics. The author is grateful to conference participants, N. Cassola, F.C. Bagliano, and R.T. Baillie for constructive comments.

${ }^{\ddagger}$ As Mitsuo Aida wrote in one of his poems, somewhere in life/ there is a path/ that must be taken regardless of how hard we try to avoid it/ at that time, all one can do is remain silent and walk the path/neither complaining nor whining/saying nothing and walking on/ just saying nothing and showing no tears/ it is then,/ as human beings, / that the roots of our souls grow deeper. This paper is dedicated to the loving memory of A. 
well independently of persistence and heteroskedasticity properties, accounting for common deterministic and stochastic factors. Monte Carlo results strongly support the proposed methodology, validating its use also for relatively small cross-sectional and temporal samples. By means of the proposed approach, new insights on US money market dynamics during the subprime and euro area financial crises are achieved. Moreover, three common factors, bearing the interpretation of level, slope and curvature factors, are extracted from the term structure of OIS spreads; we find the latter conveying additional information, relatively to commonly used credit risk measures like the TED or the BAA-AAA corporate spreads, which might be exploited, also within a composite indicator, for the construction of a risk barometer and real-time macroeconomic forecasting.

JEL classification: C22, E43, G01

Key words: long and short memory, structural breaks, common factors, principal components analysis, fractionally integrated heteroskedastic factor vector autoregressive model, subprime crisis, euro area sovereign debt crisis. 


\section{Introduction}

In the paper a general strategy for large-scale modeling of macroeconomic and financial data, set within the factor vector autoregressive model (F-VAR) framework, is proposed. ${ }^{1}$ Following the lead of dynamic factor model (DFM) analysis proposed in Geweke (1977), it is assumed that a small number of structural shocks be responsible for the observed comovement in economic data; it is also assumed that commonalities across series is described by common deterministic factors, or break processes. As the common factors are unobserved, accurate estimation may fail in the framework of small scale vector autoregressive (VAR) models, but succeed when cross-sectional information is employed to disentangle common and idiosyncratic features.

The proposed FI-HF-VAR model can be understood as a unified framework for large-scale econometric modeling, allowing for accurate investigation of cross-sectional and time series properties, independent of persistence and heteroskedasticity properties of the data, from comovement to impulse responses, forecast error variance and historical decomposition analysis. Monte Carlo results strongly support the proposed methodology.

Consistent and asymptotically normal estimation can be conjectured, as the proposed iterative multi-step estimation algorithm, similar to Stock and Watson (2005), bears the interpretation of $Q M L$ estimation, performed via the EM algorithm (Dempster et al., 1977). The iterative procedure can also be augmented by an additional step, based on the Granger and Jeon (2004) thick modelling strategy, providing median estimates of the parameters of interest and robust standard errors.

By employing a gap or cyclical representation, the proposed FI-HF-VAR model bridges the F-VAR and (the most recent) G-VAR literature, as, similarly to Dees et al. (2010) and Pesaran and Smith (2011), a weakly stationary cyclical representation is employed; yet, similarly to Bai and $\mathrm{Ng}$ (2004), both stationary and non stationary factors are allowed for, and principal components analysis (PCA), rather than cointegration analysis, is employed for the estimation of the factors. Estimation of common unobserved features by means of time domain PCA is promising, as recent asymptotic results, i.e., Bai $(2003,2004)$ and Bai and Ng (2004), have proved consistency and asymptotic normality under various conditions, covering the exact and approximate factor model case, with weakly stationary (short memory) or I(1) integrated processes, both in levels and differences, also conditionally heteroskedastic; the validity of PCA for the intermediate case of long-memory processes is also conjectured, and supporting Monte Carlo results are provided in Morana

\footnotetext{
${ }^{1}$ The literature on F-VAR models is large. See Stock and Watson (2011) for a survey.
} 
(2007) and in this study as well. Consistency and asymptotic normality for the Kalman filtering augmented PCA approach is also established by Doz et al. (2011).

The dynamic properties of US LIBOR-OIS spreads (OIS spreads) over the period May 2002 through August 2012, covering relevant events for the US money market, i.e., the subprime and euro area sovereign debt crises, are investigated by means of the proposed approach. Among the main empirical results, we find that three common components, bearing the interpretation of level, slope and curvature factors, can be extracted from the OIS spreads term structure; the latter are characterized by a deterministic trend component (break process) and strongly persistent and heteroskedastic fluctuations about trend (long memory cyclical component); two common break processes, describing the long-term evolution of OIS spreads conditional variances, bearing the interpretation of level and slope factors for the volatility term structure, are also found. Moreover, we find that the two waves of money market stress, associated with the BNP Paribas episode in August $2007^{2}$ and Lehman Brothers bankruptcy in September 2008, respectively, have lead to a wide increase in both the mean and variance OIS spreads trend levels and to a sizable increase in the persistence of money market shocks; while at the short-end of the term structure mean and variance trend components have progressively converged back to pre-crisis levels since December 2008, fluctuations about much higher mean values, than prevailing before the crisis, can be noted at its medium- to long-end, also over the postsubprime crisis period; moreover, persistence of money market shocks has not reverted back to pre-crisis intensity, actually even further increasing over the post-crisis period. A sizable increase in OIS spreads mean trend levels at the medium- to long-end of the term structure is finally associated with the beginning of the euro area sovereign debt crisis in February 2010 and its spillover to the Italian economy in September 2011.

By comparing the forward-looking properties of the OIS spreads term structure factors with alternative measures of credit/liquidity risk and financial fragility, we then find the former conveying additional information, relatively to commonly used measures like the TED or the BAA-AAA corporate spreads, which might be exploited, also within a composite indicator, for the construction of a risk barometer and real-time macroeconomic forecasting.

To our knowledge no such an in depth study on the consequences of the subprime and euro area sovereign debt crisis on the US money market has so far been contributed in the literature; the empirical results show that accu-

\footnotetext{
${ }^{2}$ On Agust 72007 the French bank BNP Paribas closed two of its investment funds, exposed to subprime mortgage risk, marking the beginning of the 2007-2009 financial crisis.
} 
rate modeling of the OIS spreads empirical properties and the understanding of the recent financial turmoil do require the comprehensive modeling framework proposed in the paper. Other empirical implementations of the proposed methodology, involving, for instance, the modeling of realized moments of financial returns and macroeconomic variables, can be envisaged, and suggestions for further applications provided as well.

After this introduction, the paper is organized as follows. In section two the econometric methodology is presented, while in section three the Monte Carlo analysis is performed; the empirical investigation of US money market dynamics during the subprime financial crisis, the ensuing recession, and the euro area sovereign debt crisis is provided in section four; finally, conclusions are drawn in section five.

\section{The FI-HF-VAR model}

The proposed modeling framework allows for different sources of persistence, in both the conditional mean and variance, for a $n$-dimensional real valued vector process $x_{t}$, covering most of the cases of interest for empirical analysis using macroeconomic and financial data. The most general specification allows for $\mathrm{I}(d)$ persistence $(0 \leq d \leq 1)$, structural breaks and common factors, both in the mean and variance. Several models are then nested in this specification, which can be derived through appropriate restrictions.

Hence, consider the following fractionally integrated heteroskedastic factor vector autoregressive (FI-HF-VAR) model

$$
\begin{aligned}
x_{t}-\Lambda_{\mu} \mu_{t}-\Lambda_{f} f_{t} & =C(L)\left(x_{t-1}-\Lambda_{\mu} \mu_{t-1}-\Lambda_{f} f_{t-1}\right)+v_{t} \\
v_{t} & \sim i i d\left(0, \Sigma_{v}\right) \\
P(L) D(L) f_{t} & =\eta_{t}=H_{t}^{1 / 2} \psi_{t} \\
\psi_{t} & \sim i i d\left(0, I_{r}\right)
\end{aligned}
$$

where $x_{t}$ is a $n$-dimensional vector of real valued integrated and heteroskedastic processes subject to structural breaks, $t=1, \ldots, T$, in deviation from the unobserved common deterministic $\left(\mu_{t}\right)$ and stochastic $\left(f_{t}\right)$ factors, where $\mu_{t}$ is a $m$-dimensional vector of common break processes, $m \leq n$, with $n \times m$ matrix of loadings $\Lambda_{\mu} ; f_{t}$ is a $r$-dimensional vector of integrated heteroskedastic common factors, $r \leq n$, of order $d_{i}$ in mean and $b_{i}$ in variance, $0 \leq d_{i} \leq 1,0 \leq b_{i} \leq 1, i=1, \ldots, r$, with $n \times r$ matrix of loadings $\Lambda_{f}$; $C(L) \equiv C_{0} L^{0}+C_{1} L+C_{2} L^{2}+\ldots+C_{s} L^{s}$ is a finite order matrix of polynomials in the lag operator with all the roots outside the unit circle, $C_{j}, j=0, . ., s$, is a square matrix of coefficients of order $n$; $v_{t}$ is a $n$-dimensional vector of 
zero mean idiosyncratic i.i.d. shocks, with contemporaneous covariance matrix $\Sigma_{v}$, assumed to be coherent with the condition of weak cross-sectional correlation of the idiosyncratic components (Assumption E) stated in Bai (2003, p.143).

Moreover, $P(L) \equiv I_{r}+P_{1} L+P_{2} L^{2}+\ldots+P_{u} L^{u}$ is a finite order matrix of polynomials in the lag operator with all the roots outside the unit circle, $P_{j}$, $j=1, . ., u$, is a square matrix of coefficients of order $r ; \psi_{t}$ is a $r$-dimensional vector of common zero mean i.i.d. shocks, with identity covariance matrix $I_{r}, E\left[\psi_{i t} v_{j s}\right]=0$ all $i, j, t, s$, respectively; $D(L)$ is a $r \times r$ diagonal matrix in the lag operator, specified according to the integration order (in mean) of the common stochastic factors, i.e.,

$$
D(L) \equiv(1-L) I_{r},
$$

for the case of $I(1)$ integration $\left(d_{i}=1\right)$;

$$
D(L) \equiv I_{r},
$$

for the $I(0)$ case or no integration (short memory) case $\left(d_{i}=0\right)$;

$$
D(L) \equiv \operatorname{diag}\left\{(1-L)^{d_{1}},(1-L)^{d_{2}}, \ldots,(1-L)^{d_{r}}\right\},
$$

for the case of fractional integration $\left(I(d)\right.$, long memory) $\left(0<d_{i}<1\right)^{3}$, where $(1-L)^{d_{i}}$ is the fractional differencing operator; the latter admits a binomial expansion, which can be compactly written in terms of the Hypergeometric function, i.e.,

$$
\begin{aligned}
(1-L)^{d_{i}} & =F\left(-d_{i}, 1,1 ; L\right) \\
& =\sum_{k=0}^{\infty} \Gamma\left(k-d_{i}\right) \Gamma(k+1)^{-1} \Gamma\left(-d_{i}\right)^{-1} L^{k} \\
& =\sum_{k=0}^{\infty} \pi_{k} L^{k},
\end{aligned}
$$

where $\Gamma(\cdot)$ is the Gamma function.

\subsection{The conditional variance process}

$H_{t}=\operatorname{Var}\left(f_{t} \mid \Omega_{t-1}\right) \equiv \operatorname{diag}\left\{h_{1, t}, h_{2, t}, \ldots, h_{r, t}\right\}$ is the $r \times r$ conditional variancecovariance matrix for the unconditionally and conditionally orthogonal common factors $f_{t}$. Consistent with the constant conditional correlation model

\footnotetext{
${ }^{3}$ See Baillie (1996) for an introduction to long memory processes.
} 
of Bollerslev (1990) and Brunetti and Gilbert (2000), the $i$ th generic element along the main diagonal of $H_{t}$ is

$$
m_{i}(L) h_{i, t}=w_{i, t}+n_{i}(L) \eta_{i, t}^{2}, \quad i=1, \ldots, r
$$

where

$$
n_{i}(L) \equiv 1-\beta_{i}(L)-\left(1-\phi_{i}(L)\right)(1-L)^{b_{i}}
$$

for the case of fractional integration (long memory) in variance $\left(0<b_{i}<1\right)$;

$$
n_{i}(L) \equiv 1-\beta_{i}(L)-\left(1-\phi_{i}(L)\right)(1-L)
$$

for the case of $I(1)$ integration in variance $\left(b_{i}=1\right)$;

$$
n_{i}(L) \equiv 1-\beta_{i}(L)-\left(1-\phi_{i}(L)\right)
$$

for the $I(0)$ or no integration (short memory) in variance case $\left(b_{i}=0\right)$; in all cases

$$
\begin{gathered}
m_{i}(L) \equiv 1-\beta_{i}(L) \\
\phi_{i}(L)=\alpha_{i}(L)+\beta_{i}(L) \\
\alpha_{i}(L) \equiv \alpha_{i, 1} L+\alpha_{i, 2} L^{2}+\ldots+\alpha_{i, q} L^{q} \\
\beta_{i}(L) \equiv \beta_{i, 1} L+\beta_{i, 2} L^{2}+\ldots+\beta_{i, p} L^{p},
\end{gathered}
$$

and all the roots of the $\phi_{i}(L)$ and $\beta_{i}(L)$ polynomials are outside the unit circle.

A factor structure in the $w_{i, t}$ component may also be allowed for by writing

$$
w_{i, t}=\Lambda_{g, i} g_{t},
$$

where $g_{t}$ is a $l$-dimensional vector of common break processes in variance, $l \leq r$, with $r \times l$ matrix of loadings $\Lambda_{g}$, and $\Lambda_{g, i}$ being its $i$ th row.

The conditional variance process $h_{i, t} \equiv \operatorname{Var}\left(f_{i, t} \mid \Omega_{i, t-1}\right), i=1, \ldots, r$, is therefore of the FIGARCH $\left(p, b_{i}, z\right)$ type (Baillie et al., 1996), with $z=$ $\max \{p, q\}$ or the IGARCH $(p, q)$ type (Engle and Bollerslev, 1986) for the fractionally integrated and integrated case, respectively, and of the $G A R C H(p, q)$ type (Bollerslev, 1986) for the non integrated case, augmented by a timevarying intercept $w_{i, t}$ in the conditional variance equation.

Different specifications for $w_{i, t}$ have been suggested in the literature, i.e., a spline function (Engle and Rangel, 2008), a Gallant (1984) flexible functional form (Baillie and Morana, 2009), a spline-dummy function (Cassola 
and Morana, 2012), a general smooth transition logistic function (Amado and Terasvirta, 2008), a Markov switching mechanism (Hamilton and Susmel, 2004); in all cases alternating regimes, recurrent or not recurrent, are allowed for in the conditional variance equations. The above mentioned mechanisms, may also be implemented for the modelling of structural breaks in the mean of the processes $\left(\mu_{t}\right)$; see Hamilton (1989), Enders and Lee (2004), Gonzalez et al. (2009), Cassola and Morana (2012), Baillie and Morana (2012).

The following $A R C H(\infty)$ representation can be obtained from each of the three above models

$$
\begin{aligned}
h_{i, t} & =\frac{w_{i, t}}{m_{i}(1)}+\frac{n_{i}(L)}{m_{i}(L)} \eta_{i, t}^{2} \quad i=1, \ldots, r \\
& =w_{i, t}^{*}+\psi_{i}(L) \eta_{i, t}^{2}
\end{aligned}
$$

where $w_{i, t}^{*}=\frac{w_{i, t}}{m_{i}(1)}$ and $\psi_{i}(L)=\frac{n_{i}(L)}{m_{i}(L)}=\psi_{1, i} L+\psi_{2, i} L^{2}+\ldots$

The term $w_{i, t}^{*}$ then bears the interpretation of break in variance process, or time-varying unconditional variance process (no integration case), or longterm conditional variance level (unit root and fractional integration cases).

To guarantee the non negativity of the conditional variance process at each point in time all the coefficients in the $A R C H(\infty)$ representation must be non-negative, i.e., $\psi_{j, i} \geq 0$ for all $j \geq 1$ and $w_{i, t}^{*}>0$ for any $t$. Sufficient conditions, for various parameterization, can be found in Baillie et al. (1996), Engle and Bollerslev (1986), Bollerslev (1986), Baillie and Morana (2009), Conrad and Hag (2006), and Chung (1999).

\subsection{Examples of nested models}

From (1) and (2), by setting $D(L)=I_{r}, H_{t}^{1 / 2}=\Sigma_{\eta}^{1 / 2}, p=m, \mu_{t}=\mu, \Lambda_{\mu}=I_{n}$, the $\mathrm{I}(0)$ homoskedastic F-VAR $(s, u)$ model

$$
\begin{aligned}
x_{t}-\mu-\Lambda_{f} f_{t} & =C(L)\left(x_{t-1}-\mu-\Lambda_{f} f_{t-1}\right)+v_{t} \\
v_{t} & \sim i i d\left(0, \Sigma_{v}\right) \\
P(L) f_{t} & =\eta_{t}, \\
\eta_{t} & \sim \operatorname{iid}\left(0, \Sigma_{\eta}\right)
\end{aligned}
$$

is then obtained; moreover, by allowing $H_{t}^{1 / 2}$ to evolve according to $(4),(7)$, and (8)-(11), with $w_{i, t}=w_{i}, m_{i}(L) \equiv 1-\beta_{i} L$ and $\phi_{i}(L)=\alpha_{i} L+\beta_{i} L$, the $\mathrm{I}(0)$ $\mathrm{F}-\operatorname{VAR}(s, u)$-GARCH$(1,1)$ model is obtained; also, by assuming $(5)$ rather then $(7)$, the $\mathrm{I}(0) \mathrm{F}-\operatorname{VAR}(s, u)-\mathrm{FIGARCH}(1, b, 1)$ is obtained. Applications of 
the latter models for the modeling of macroeconomic variables in stationary form and financial returns may be envisaged. See, for instance, Morana (2012) for a large-scale application to the modeling of the global economy and the macro-finance interface.

Moreover, by setting $D(L) \equiv(1-L) I_{r}$, the $\mathrm{I}(1) \operatorname{F-VAR}(s, u)$ model

$$
\begin{aligned}
x_{t}-\mu-\Lambda_{f} f_{t} & =C(L)\left(x_{t-1}-\mu-\Lambda_{f} f_{t-1}\right)+v_{t} \\
v_{t} & \sim i i d\left(0, \Sigma_{v}\right) \\
P(L)(1-L) f_{t} & =\eta_{t} \\
\eta_{t} & \sim \operatorname{iid}\left(0, \Sigma_{\eta}\right)
\end{aligned}
$$

is obtained, as well as, by, imposing the same restrictions as above, the $\mathrm{I}(1)$ $\operatorname{F-VAR}(s, u)$-GARCH$(1,1)$ and F-VAR $(s, u)$-FIGARCH $(1, b, 1)$ models. Applications of the latter models for the modeling of non-stationary macroeconomic variables and financial asset prices may be envisaged.

In addition to interest rate spreads term structure modeling, other applications of the most general framework contributed in the paper may also be envisaged, as for instance for the modeling of inflation rates and realized moments of financial returns.

\subsection{The reduced fractional VAR form}

Depending on persistence properties of the data, the vector autoregressive representation (VAR) for the factors $f_{t}$ and the series $x_{t}$ can be written as follows:

i) for the case of fractional integration (long memory) $\left(0<d_{i}<1\right)$, by taking into account the binomial expansion in (3), it follows $P(L) D(L) \equiv$ $I-\Pi(L), \Pi(L)=\Pi_{1} L^{1}+\Pi_{2} L^{2}+\ldots$, where $\Pi_{i}, i=1,2, \ldots$, is a square matrix of coefficients of dimension $r$; by substituting (2) into (1), the infinite order vector autoregressive representation for the factors $f_{t}$ and the series $x_{t}$ can then be written as

$$
\begin{aligned}
& {\left[\begin{array}{c}
f_{t} \\
x_{t}-\Lambda_{\mu} \mu_{t}
\end{array}\right]=} {\left[\begin{array}{cc}
\Pi_{f}^{*}(L) & 0 \\
\Pi^{*}(L) & C(L)
\end{array}\right]\left[\begin{array}{c}
f_{t-1} \\
x_{t-1}-\Lambda_{\mu} \mu_{t-1}
\end{array}\right] } \\
&+\left[\begin{array}{c}
\eta_{t} \\
\varepsilon_{t}
\end{array}\right] \\
& {\left[\begin{array}{c}
\eta_{t} \\
\varepsilon_{t}
\end{array}\right]=\left[\begin{array}{c}
I \\
\Lambda_{f}
\end{array}\right]\left[{\sqrt{h_{t}}}^{\prime} \psi_{t}\right]+\left[\begin{array}{c}
0 \\
v_{t}
\end{array}\right] }
\end{aligned}
$$


where $\Pi_{f}^{*}(L)=\Pi(L) L^{-1}$ and $\Pi^{*}(L)=\left[I_{n}-C(L) L\right] \Lambda_{f} \Pi(L) L^{-1}$; since the infinite order representation cannot be handled in estimation, a truncation to a suitable large lag for the polynomial matrix $\Pi(L)$ is required. ${ }^{4}$ Hence, $\Pi(L)=\sum_{j=1}^{p^{*}} \Pi_{j} L^{j} ;$

ii) for the case of integration $\left(d_{i}=1\right)$, it should be firstly recalled that

$$
\begin{aligned}
P(L) D(L) & \equiv P(L)(1-L) \\
& \equiv\left(I_{r}-\rho L\right)-\left(P_{1} L+P_{2} L^{2}+\ldots+P_{u} L^{u}\right)(1-L)
\end{aligned}
$$

with $\rho=I_{r}$.

The latter may be rewritten in the equivalent polynomial matrix form

$$
I_{r}-\Gamma_{1} L-\Gamma_{2} L^{2}-\ldots-\Gamma_{u+1} L^{u+1}
$$

where $\Gamma_{i}, i=1, \ldots, u+1$, is a square matrix of coefficients of dimension $r$, and

$$
\begin{gathered}
\Gamma_{1}+\Gamma_{2}+\ldots+\Gamma_{u+1}=\rho=I_{r} \\
P_{i}=-\left(\Gamma_{i+1}+\Gamma_{i+2}+\ldots+\Gamma_{u+1}\right), \quad i=1,2, \ldots, u .
\end{gathered}
$$

Then, the (finite order) vector autoregressive representation for the factors $f_{t}$ and the series $x_{t}$ can be written as in $(19)$, with $\Pi_{f}^{*}(L)=\Gamma(L) L^{-1}$ and $\Pi^{*}(L)=\left[I_{n}-C(L) L\right] \Lambda_{f} \Gamma(L) L^{-1}$;

iii) for the case of no integration (short memory) $\left(d_{i}=0\right)$, the (finite order) vector autoregressive representation for the factors $f_{t}$ and the series $x_{t}$ can still be written as in (19), but recalling that $D(L) \equiv I_{r}$, and therefore $P(L) D(L)=P(L)$, then, $\Pi_{f}^{*}(L)=P(L) L^{-1}$ and $\Pi^{*}(L)=$ $\left[I_{n}-C(L) L\right] \Lambda_{f} P(L) L^{-1}$.

\footnotetext{
${ }^{4}$ Monte Carlo evidence reported in Chan and Palma (1998) suggests that the truncation lag should increase with the sample size and the complexity of the ARFIMA representation of the long memory process, still remaining very small relatively to the sample size. For instance, for the covariance stationary fractional white noise case and a sample of 100 observations truncation can be set as low as 6 lags, while for a sample of 10,000 observations it should be increased to 14 lags; for the case of a covariance stationary ARFIMA $(1, \mathrm{~d}, 1)$ process and a sample of 1,000 observations truncation may be set to 30 lags.
} 


\subsection{Estimation}

Estimation of the model can be implemented following a multi-stage iterative procedure, similar to Stock and Watson (2005), consisting of the following steps.

- Step 1: persistence analysis. Stationarity/non stationarity tests and structural break tests are carried out on the series of interest $\left(x_{t}\right)$ in order to determine their persistence properties, i.e., deterministic and/or stochastic.

At this stage each component $x_{i, t}, i=1, \ldots, n$, of the vector time series $x_{t}$ is decomposed into its purely deterministic (trend/break process; $\hat{b}_{i, t}$ ) and purely stochastic (break-free, $\hat{l}_{i, t}=x_{i, t}-\hat{b}_{i, t}$ ) parts. As neglected structural breaks may lead to processes which appear to show persistence of the long memory or unit root type (Perron, 1989; Granger and Hyung, 2004; Diebold and Inoue, 2001), procedures allowing for structural breaks when testing for the integration order should be employed (Baillie and Morana, 2009, 2012; Beckers et al., 2006; Enders and Lee, 2005; Perron, 1989; McCloskey and Perron, 2011; Phillips and Shimotsu, 2004; Dolado et al., 2005; Perron and Qu, 2010; Ohanissian et al., 2008; Shimotsu, 2006; Morana, 2009). An estimate of the stochastic persistence parameter is however only required at Step 4, where the analysis is performed on each of the common factors, as in Bai and $\mathrm{Ng}(2004) .^{5}$

- Step 2: initialization. The initialization stage yields an initial estimate of the common deterministic (break processes) $\left(\mu_{t}\right)$ and stochastic factors $\left(f_{t}\right)$ and the $C(L)$ polynomial matrix, i.e., an initial estimate of the equation system in (1).

•• Hence, the initial estimate of the $m \leq n$ common break processes is obtained by means of Principal Components Analysis (PCA) implemented using the estimated break process $\hat{b}_{i, t}, i=1, \ldots, n$, collected in the vector $\hat{b}_{t}$, yielding $\hat{\mu}_{*, t} \equiv H_{\mu} \hat{\mu}_{t}=\hat{\Lambda}_{b}^{-1 / 2} \hat{A}^{\prime} \hat{b}_{t}$, where $\hat{\Lambda}_{b}$ is the $m \times m$ diagonal matrix of the non zero eigenvalues of $\hat{\Sigma}_{\hat{b}}$, the estimated reduced rank $(m<n)$ $n \times n$ variance-covariance matrix of the (estimated) break processes $\hat{b}_{i, t}, \hat{A}$ is the $n \times m$ matrix of the associated orthogonal eigenvectors, and $H_{\mu}$ is an invertible square matrix of order $m$.

- Next, the initial estimate of the $r \leq n$ common stochastic factors is obtained by means of PCA implemented using the estimated break-free series

\footnotetext{
${ }^{5}$ While from a theoretical point of view a linear combination of processes with different orders of integration is integrated of order equal to the highest one among those shown by the processes involved in the linear combination, empirically it can be difficult to accurately determine the order of integration of a linear combination process, depending on the relative variance of the various components. Note that the proposed approach is not affected by this latter drawback.
} 
$\hat{l}_{i, t}, i=1, \ldots, n$, collected in the vector $\hat{l}_{t}$, yielding $\hat{f}_{*, t} \equiv H_{f} \hat{f}_{t}=\hat{\Lambda}_{l}^{-1 / 2} \hat{B}^{\prime} \hat{l}_{t}$, where $\hat{\Lambda}_{l}$ is the $r \times r$ diagonal matrix of the non zero eigenvalues of $\hat{\Sigma}_{\hat{l}}$, the estimated reduced rank $(r<n) n \times n$ variance-covariance matrix of the (estimated) break-free processes $\hat{l}_{t}, \hat{B}$ is the $n \times r$ matrix of the associated orthogonal eigenvectors and $H_{f}$ is an invertible square matrix of order $r$.

- Finally, conditional on the initial estimate of the common deterministic and stochastic factors, the initial estimate of the polynomial matrix $C(L)$ and $\Lambda_{f}$ and $\Lambda_{\mu}$ factor loading matrices is obtained by means of OLS estimation of the equation system in (1). This can be obtained by first (OLS) regressing the actual series $x_{t}$ on the estimated common break processes and stochastic factors to obtain $\hat{\Lambda}_{f}$ and $\hat{\Lambda}_{\mu}$; alternatively, $\hat{\Lambda}_{\mu}=\hat{A} \hat{\Lambda}_{b}^{1 / 2}$ and $\hat{\Lambda}_{f}=\hat{B} \hat{\Lambda}_{l}^{1 / 2}$, as yield by PCA; then, the gap vector is computed as $x_{t}-\hat{\Lambda}_{\mu} \hat{\mu}_{*, t}-\hat{\Lambda}_{f} \hat{f}_{*, t}$, and $\hat{C}(L)$ is obtained by means of OLS estimation of the VAR model in (1).

Several criteria are available for the selection of the number of common stochastic factors, ranging from heuristic or statistical eigenvalue-based approaches (Jackson, 1993; Kapetanios, 2010; Cragg and Donald, 1997; Gill and Lewbel, 1992, Robin and Smith, 2000), to the variance test of Connor and Korajczyk (1993), and the more recent information criteria (Stock and Watson, 1998; Forni et al., 2000; Bai and Ng, 2002) and "primitive" shock (Bai and Ng, 2007; Stock and Watson, 2005) based procedures.

- Step 3: the iterative procedure. An updated estimate of the equation system in (1) is obtained as follows.

- First, a new estimate of the $m$ common deterministic factors, and their factor loading matrix, can be obtained by the application of PCA to the (new) stochastic factor-free series $x_{t}-[I-\hat{C}(L) L] \hat{\Lambda}_{f} \hat{f}_{*, t}$, yielding $\hat{\Lambda}_{\mu}^{(\text {new })}$ and $\hat{\mu}_{*, t}^{(n e w)} 6$

-• Next, conditional on the new common break processes and their factor loading matrix, the new estimate of the common long memory factors is obtained from the application of PCA to the (new) break-free processes $\hat{l}_{t}^{(n e w)}=x_{t}-\hat{\Lambda}_{\mu}^{(n e w)} \hat{\mu}_{*, t}^{(n e w)}$, yielding $\hat{\Lambda}_{f}^{(n e w)}$ and $\hat{f}_{*, t}^{(n e w)} .^{7}$

-• Finally, conditional on the new estimated common break processes and long memory factors, the new estimate of the gap vector $x_{t}-\hat{\Lambda}_{\mu}^{(n e w)} \hat{\mu}_{* . t}^{(n e w)}$ $\hat{\Lambda}_{f}^{(n e w)} \hat{f}_{*, t}^{(n e w)}$ is obtained, and the new estimate $\hat{C}(L)^{(n e w)}$ can be computed

${ }^{6}$ Alternatively, $\hat{\Lambda}_{\mu}^{(n e w)}$ can be obtained by regressing $x_{t}$ on $\hat{\mu}_{*, t}^{(n e w)}$ (and the initial estimate $\hat{f}_{*, t}$ ), using OLS.

${ }^{7}$ Alternatively, $\hat{\Lambda}_{f}^{(n e w)}$ can be obtained by regressing $x_{t}$ on $\hat{f}_{*, t}^{(n e w)}$ (and the updated estimate $\hat{\mu}_{*, t}^{(n e w)}$ ), using OLS. This would also yield a new estimate $\hat{\Lambda}_{\mu}^{(n e w)}$ to be used in the computation of the updated gap vector. 
by means of OLS estimation of the VAR model in (1).

- The procedure described in step 3 is iterated until convergence, yielding the final estimates $\hat{\Lambda}_{\mu}^{(f i n)}, \hat{\mu}_{*, t}^{(f i n)}, \hat{\Lambda}_{f}^{(f i n)}, \hat{f}_{*, t}^{(f i n)}$, and $\hat{C}(L)^{(f i n)}$.

Note that the proposed iterative procedure bears the interpretation of $Q M L$ estimation performed by means of the EM algorithm. In the $E$-step the unobserved factors are estimated, given the observed data and the current estimate of model parameters, by means of $P C A$; in the $M$-step the likelihood function is maximized (OLS estimation of the $C(L)$ matrix is performed) under the assumption that the unobserved factors are known, conditioning on their E-step estimate. Convergence to the one-step $Q M L$ estimate is ensured, as the value of the likelihood function is increased at each step. Note that the Expectation step of the EM algorithm relies on consistent estimation of the unobserved components by means of PCA. In this respect it should be noted that $\min \{\sqrt{n}, \sqrt{T}\}$ consistency and asymptotic normality of PCA for $\mathrm{I}(0)$ and $\mathrm{I}(1)$ (non cointegrated) unobserved common factors has been established in Bai $(2003,2004)$ under general conditions. While there are no asymptotic results for the application of PCA to fractionally integrated and trend stationary processes, supporting Monte Carlo evidence is provided by Morana (2007) and in this study. ${ }^{8}$ Moreover, likewise in the Maximization step of the EM algorithm, $\sqrt{T}$ consistent and asymptotically normal estimation of the polynomial matrix $C(L)$ is yield by OLS estimation of the VAR model for the $\mathrm{I}(0)$ gap series, holding the latter as they were actually observed. As shown by Bai and Ng $(2006,2008)$, when the unobserved factors are estimated by means of PCA in the $E$-step, the generated regressors problem is in fact not an issue for consistent estimation in the $M$-step, due to faster vanishing of the estimation error, provided $\sqrt{T} / N \rightarrow 0$ for linear models, and $T^{5 / 8} / N \rightarrow 0$ for non linear models.

- Step 4: restricted estimation of the full model. Once the final estimate of the equation system in (1) is available, the reduced VAR form in (19) is estimated as follows:

i) for the case of fractional integration (long memory) $\left(0<d_{i}<1\right)$, the fractional differencing parameter is (consistently) estimated first, for each component of the common factor vector $\hat{f}_{*, t}^{(f i n)}$, yielding the estimates $\hat{d}_{i}$, $i=1, \ldots, r$, collected in $\hat{D}(L)$ matrix; then, $\hat{P}(L)$ is obtained by means of OLS estimation of the $V A R(u)$ model for the (estimated) fractionally differenced common long memory factors $\left(\hat{D}(L) \hat{f}_{*, t}^{(f i n)}\right)^{9}$; hence, $I-\hat{\Pi}(L)=$

\footnotetext{
${ }^{8}$ The use of PCA for the estimation of common deterministic trends has previously been advocated by Bierens (2000). Yet, details cannot be found in the published version of his paper.

${ }^{9}$ Alternatively, for the covariance stationary long memory case only, consistent and
} 
$\hat{P}(L) \hat{D}^{*}(L)$, where $\hat{D}^{*}(L)$ is the $r$-dimensional diagonal polynomial matrix in the lag operator containing the $p$ th order $(p>u)$ truncated binomial expansion of the elements in $\hat{D}(L)$. Then, $\hat{\Pi}_{f}^{*}(L)=\hat{\Pi}(L) L^{-1}$ and $\hat{\Pi}^{*}(L)=$ $\left[I_{n}-\hat{C}(L)^{(f i n)} L\right] \hat{\Lambda}_{f}^{(f i n)} \hat{\Pi}(L) L^{-1}$

ii) for the $\mathrm{I}(1)$ case $\left(d_{i}=1\right), \hat{\Pi}_{f}^{*}(L)=\hat{\Gamma}(L) L^{-1}$, where $\hat{\Gamma}(L)$ is obtained by means of OLS estimation of the $V A R(u+1)$ model for the (estimated) common stochastic factors $\left(\hat{f}_{*, t}^{(f i n)}\right)$; then $\hat{\Pi}^{*}(L)=\left[I_{n}-\hat{C}(L)^{(f i n)} L\right] \hat{\Lambda}_{f}^{(f i n)} \hat{\Gamma}(L) L^{-1}$;

iii) for the case of no integration (short memory) $\left(d_{i}=0\right), \hat{\Pi}_{f}^{*}(L)=$ $\hat{P}(L) L^{-1}$ where $\hat{P}(L)$ is obtained by means of OLS estimation of the $V A R(u)$ model for the (estimated) common stochastic factors $\left(\hat{f}_{*, t}^{(f i n)}\right)$; then $\hat{\Pi}^{*}(L)=$ $\left[I_{n}-\hat{C}(L)^{(f i n)} L\right] \hat{\Lambda}_{f}^{(f i n)} \hat{P}(L) L^{-1}$.

By taking the estimated factors (and fractional differencing parameter) as they were actually observed, again standard asymptotic theory would then apply. The VAR representation of the FI-HF-VAR model can then be inverted (see the next section for details) and impulse response and forecast error variance decomposition analysis performed.

- Step 5: simulation. Following the thick modelling strategy of Granger and Jeon (2004), a further step can be added to the procedure, in order to compute median estimates of the parameters of interest, impulse responses and forecast error variance decomposition, as well as their confidence intervals.

- Step 6: conditional variance analysis. Conditional variance analysis can be carried out using a procedure similar to the O-GARCH model of Alexander (2002):

i) firstly, conditional variance estimation is carried out factor by factor, using the estimated factor residuals $\hat{\eta}_{t}$, yielding $\hat{h}_{i, t}, i=1,2, \ldots, r ; Q M L$ estimation can be performed in a variety of settings, ranging from standard GARCH $(p, q)$ (Bollerslev, 1986) and FIGARCH $(p, b, z)$ (Baillie et al., 1996) models to their "adaptive" generalizations (Engle and Rangel, 2008; Baillie and Morana, 2009; Amado and Terasvirta, 2008; Hamilton and Susmel, 2004 ), in order to allow for different sources of persistence in variance;

ii) secondly, consistent with the assumptions of conditional and unconditional orthogonality of the factors, the conditional variance-covariance $\left(H_{x, t}\right)$

asymptotically normal estimation of the VARFIMA model can be obtained by means of Conditional-Sum-of-Squares (Robinson, 2006), exact Maximum Likelihood (Sowell, 1992), or Indirect (Martin and Wilkins, 1999) estimation. See also Baillie and Morana (2012). 
and correlation $\left(R_{x, t}\right)$ matrices for the actual series may be estimated as

$$
\begin{aligned}
\hat{H}_{x, t} & =\hat{\Lambda}_{f} \hat{H}_{t} \hat{\Lambda}_{f}^{\prime}+\hat{\Sigma}_{v} \\
\hat{R}_{x, t} & =\hat{H}_{x, t}^{*-1 / 2} \hat{H}_{x, t} \hat{H}_{x, t}^{*-1 / 2},
\end{aligned}
$$

where $\hat{H}_{t}=\operatorname{diag}\left\{\hat{h}_{1, t}, \hat{h}_{2, t}, \ldots, \hat{h}_{r, t}\right\}$ and $\hat{H}_{x, t}^{*}=\operatorname{diag}\left\{\hat{h}_{x_{1}, t}, \hat{h}_{x_{2}, t}, \ldots, \hat{h}_{x_{n}, t}\right\}$.

Relaxing the assumption of conditional hortogonality is also feasible in the proposed framework, as the dynamic conditional covariances, i.e., the off-diagonal elements in $H_{t}$, can be obtained, after step $i$ ) above, by means of the second step in the estimation of the Dynamic Conditional Correlation model (DCC; Engle, 2002) or the Dynamic Equicorrelation model (DECO; Engle and Kelly, 2008).

\subsubsection{Reduced form and structural vector moving average repre- sentation of the FI-HF-VAR model}

In the presence of unconditional heteroskedasticity, the computation of the impulse response functions and the forecast error variance decomposition (FEVD) should be made dependent on the estimated unconditional variance for each regime. In the case of (continuously) time-varying unconditional variance, policy analysis may then be computed at each point in time. For some of the conditional variance models considered in the paper, i.e., the FIGARCH and IGARCH processes, the population unconditional variance does not actually exist; in the latter cases the $w_{i, t}$ component just bears the interpretation of long term level for the conditional variance; policy analysis is still feasible, yet subject to a different interpretation, FEVD referring, for instance, not to the proportion of forecast error (unconditional) variance accounted for by each structural shock, but to the proportion of forecast error (conditional) long term variance accounted for by each structural shock. With this caveat in mind, the actual computation of the above quantities is achieved in the same way as in the case of well defined population unconditional variance.

Hence, the computation of the vector moving average (VMA) representation for the FI-HF-VAR model depends on the persistence properties of the data. The following distinctions should then be made.

For the short memory case, i.e., the zero integration order case $\left(d_{i}=0\right)$, the VMA representation for the $x_{t}-\Lambda_{\mu} \mu_{t}$ process can be written as

$$
x_{t}-\Lambda_{\mu} \mu_{t}=G(L) \eta_{t}+F(L) v_{t},
$$

where $G(L) \equiv \Lambda_{f} P(L)^{-1}$ and $F(L) \equiv[I-C(L) L]^{-1}$. 
For the long memory case $\left(0<d_{i}<1\right)$ and the case of $\mathrm{I}(1)$ non stationarity $\left(d_{i}=1\right)$, the VMA representation should be computed for the differenced process $(1-L)\left(x_{t}-\Lambda_{\mu} \mu_{t}\right)$ yielding

$$
(1-L)\left(x_{t}-\Lambda_{\mu} \mu_{t}\right)=G(L)^{+} \eta_{t}+F(L)^{+} v_{t}
$$

where $G(L)^{+} \equiv \Lambda_{f}(1-L) P(L)^{-1}=(1-L) G(L)$ and $F(L)^{+} \equiv(1-L)[I-C(L) L]^{-1}=$ $(1-L) F(L)$.

Moreover, for the long memory case, the generic lag polynomial element in $G(L)^{+}$, i.e., $g_{i}(L)^{+}$, can be written in terms of the Hypergeometric function

$$
\begin{aligned}
g_{i}(L)^{+} & =F\left(d_{i}-1,1,1 ; L\right) \\
& =\sum_{k=0}^{\infty} \Gamma\left(k+d_{i}\right) \Gamma(k+1)^{-1} \Gamma\left(d_{i}\right)^{-1} L^{k} \\
& =\sum_{k=0}^{\infty} \lambda_{k} L^{k} .
\end{aligned}
$$

Impulse responses for the $x_{t}-\Lambda_{\mu} \mu_{t}$ process can then be finally computed as $I+\sum_{j=1}^{k} G_{j}^{+}$and $I+\sum_{j=1}^{k} F_{j}^{+}, k=1,2, \ldots$

Identification of structural shocks The identification of the structural shocks in the FI-HF-VAR model can be implemented in two steps. Firstly, denoting by $\xi_{t}$ the vector of the $r$ structural common factor shocks, the relation between reduced and structural form common shocks can be written as $\xi_{t}=H \eta_{t}$, where $H$ is square and invertible. Therefore, the identification of the structural common factor shocks amounts to the estimation of the elements of the $H$ matrix. It is assumed that $E\left[\xi_{t} \xi_{t}^{\prime}\right]=I_{r}$, and hence $H \Sigma_{\eta} H^{\prime}=I_{r}$. As the number of free parameters in $\Sigma_{\eta}$ is $r(r+1) / 2$, at most $r(r+1) / 2$ parameters in $H^{-1}$ can be uniquely identified through the $\Sigma_{\eta}=H^{-1} H^{\prime-1}$ system of nonlinear equations in the unknown parameters of $H^{-1}$. Additional $r(r-1) / 2$ restrictions need then to be imposed for exact identification of $\mathrm{H}^{-1}$, by constraining the contemporaneous or long-run responses to structural shocks; for instance, recursive (Choleski) or non recursive structures can be imposed on the VAR model for the common factors through exclusion or linear/non-liner restrictions, as well as sign restrictions, on the contemporaneous impact matrix $H^{-1} \cdot{ }^{10}$

Secondly, by denoting $\psi_{t}$ the vector of $n$ structural idiosyncratic disturbances, the relation between reduced form and structural form idiosyncratic

\footnotetext{
${ }^{10}$ See Kilian (2011) for a recent survey.
} 
shocks can be written as $\psi_{t}=\Theta v_{t}$, where $\Theta$ is square and invertible. Hence, the identification of the structural idiosyncratic shocks amounts to the estimation of the elements of the $\Theta$ matrix. It is assumed that $E\left[\psi_{t} \psi_{t}^{\prime}\right]=I_{n}$, and hence $\Theta \Sigma_{v} \Theta^{\prime}=I_{n}$. Then, in addition to the $n(n+1) / 2$ equations provided by $\Sigma=\Theta^{-1} \Theta^{\prime-1}, n(n-1) / 2$ restrictions need to be imposed for exact identification of $\Theta^{-1}$, similarly to what required for the common structural shocks.

Note that preliminary to the estimation of the $\Sigma_{v}$ matrix, $\hat{v}_{t}$ should be obtained from the residuals of an OLS regression of $\hat{\varepsilon}_{t}$ on $\hat{\eta}_{t}$; the latter operation would grant orthogonality between common and idiosyncratic residuals.

The structural VMA representation can then be written as

$$
x_{t}-\Lambda_{\mu} \mu_{t}=G^{*}(L) \xi_{t}+F^{*}(L) \psi_{t},
$$

where $G^{*}(L)=G(L) H^{-1}, F^{*}(L)=F(L) \Theta^{-1}$, or

$$
(1-L)\left(x_{t}-\Lambda_{\mu} \mu_{t}\right)=G^{\circ}(L) \xi_{t}+F^{\circ}(L) \psi_{t},
$$

where $G^{\circ}(L)=G^{+}(L) H^{-1}, F^{\circ}(L)=F(L)^{+} \Theta^{-1}$, according to persistence properties, and $E\left[\psi_{i, t} \xi_{j, t}^{\prime}\right]=0$ any $i, j$.

\section{Monte Carlo analysis}

Consider the following data generation process (DGP) for the $N$-dimensional vector process $x_{t}$

$$
\begin{aligned}
x_{t}-\Lambda_{\mu} \mu_{t}-\Lambda_{f} f_{t} & =C\left(x_{t-1}-\Lambda_{\mu} \mu_{t-1}-\Lambda_{f} f_{t-1}\right)+v_{t} \\
v_{t} & \sim i i d\left(0, \sigma^{2} I_{N}\right),
\end{aligned}
$$

where $C$ is a $N \times N$ matrix of coefficients, $\Lambda_{\mu}$ and $\Lambda_{f}$ are $N \times 1$ vectors of loadings, and $\mu_{t}$ and $f_{t}$ are the common deterministic and long memory factors, respectively, at time period $t$, with

$$
(1-\phi L)(1-L)^{d} f_{t}=\eta_{t}
$$

Then, for the conditionally heteroskedastic case it is assumed

$$
\begin{gathered}
\eta_{t}=\sqrt{h_{t}} \psi_{t} \quad \psi_{t} \sim \operatorname{iid}(0,1) \\
{[1-\alpha L-\beta L](1-L)^{b}\left(\eta_{t}^{2}-\sigma_{\eta}^{2}\right)=[1-\beta L]\left(\eta_{t}^{2}-h_{t}\right),}
\end{gathered}
$$


while

$$
\eta_{t} \sim \operatorname{iid}(0,1)
$$

for the conditionally homoskedastic case.

Different values for the autoregressive idiosyncratic parameter $\rho$, common across the $N$ cross-sectional units $\left(C=\rho I_{N}\right)$, have been considered, i.e., $\rho=$ $\{0,0.2,0.4,0.6,0.8\}$, as well as for the fractionally differencing parameter $d=$ $\{0,0.2,0.4,0.6,0.8,1\}$ and the common factor autoregressive parameter $\phi$, setting $\phi=\{0.2,0.4,0.6,0.8\}$ for the non integrated case and $\phi=\{0, d / 2\}$ for the fractionally integrated and integrated cases; $\phi>\rho$ is always assumed in the experiment. For the conditional variance equation it is assumed $\alpha=0.05$ and $\beta=0.90$ for the short memory case, and $\alpha=0.05, \beta=0.30$ and $b=0.45$ for the long memory case. The inverse signal to noise ratio is given by $\sigma^{2} / \sigma_{\eta}^{2}$, taking values $\sigma^{2} / \sigma_{\eta}^{2}=\{4,2,1,0.5,0.25\}$. Finally, $\Lambda_{\mu}$ and $\Lambda_{f}$ are set equal to unitary vectors.

Moreover, in addition to the structural stability case, i.e., $\mu_{t}=\mu=0$, two designs with breaks have been considered for the component $\mu_{t}$, i.e.,

$i$ ) the single step change in the intercept at the midpoint of the sample case, i.e.,

$$
\mu_{t}=\left\{\begin{array}{rr}
0 & t=1, \ldots, T / 2 \\
4 & t=T / 2+1, \ldots, T
\end{array} ;\right.
$$

ii) the two step changes equally spaced throughout the sample case, with the intercept increasing at one third of the way through the sample and then decreasing at a point two thirds of the length of the sample, i.e.,

$$
\mu_{t}=\left\{\begin{array}{cr}
0 & t=1, \ldots, T / 3 \\
4 & t=T / 3+1, \ldots, 2 T / 3 \\
2 & t=2 T / 3+1, \ldots, T
\end{array} .\right.
$$

The sample size investigated is $T=100,500$, and the number of crosssectional units is $N=30$. For the no breaks case also other cross-sectional sample sizes have been employed, i.e., $N=5,10,15,50$.

The number of replications has been set to 2,000 for each case.

The performance of the proposed multi-step procedure has then been assessed with reference to the estimation of the unobserved common stochastic and deterministic factors, and the $\phi$ and $\rho$ autoregressive parameters. Being not contributed by the proposed approach, the location of the break points and the value of the fractional differencing parameter are taken as known.

Concerning the estimation of the common factors the Theil's inequality coefficient $(I C)$ and the correlation coefficient (Corr) have then been em- 
ployed in the evaluation, i.e.,

$$
\begin{aligned}
I C & =\sqrt{\frac{1}{T} \sum_{t=1}^{T}\left(z_{t}-\hat{z}_{t}\right)^{2}} /\left(\sqrt{\frac{1}{T} \sum_{t=1}^{T} z_{t}^{2}}+\sqrt{\left.\frac{1}{T} \sum_{t=1}^{T} \hat{z}_{t}^{2}\right)},\right. \\
\operatorname{Corr} & =\operatorname{Cov}\left(z_{t}, \hat{z}_{t}\right) / \sqrt{\operatorname{Var}\left(z_{t}\right) \operatorname{Var}\left(\hat{z}_{t}\right)},
\end{aligned}
$$

where $z_{t}=\mu_{t}, f_{t}$ is the true unobserved component and $\hat{z}_{t}$ its estimate. The above statistics have been computed for each Monte Carlo replication and then averaged.

\subsection{Results}

The results for the non integration case are reported in Figures 1-2 (and 5, columns 1 and 3), while Figures 3-4 (and 5, columns 2 and 4) refer to the fractionally integrated and integrated cases (the integrated case, independent of the type of integration, thereafter). In all cases results refer to the estimated parameters for the first equation in the model. Since the results are virtually unaffected by the presence of conditional heteroskedasticity, for reasons of space, only the heteroskedastic case is discussed. Moreover, only the results for the $\phi=d / 2$ case are reported for the integrated case, as similar results have been obtained for the $\phi=0$ case. $^{11}$

\subsubsection{The structural stability case}

As is shown in the plots, for a cross-sectional sample size $N=30$ units, a negligible downward bias for the $\rho$ parameter can be noted $(-0.02$ and -0.03 , on average, for the non integrated and integrated case, respectively, and $T=100 ;-0.01$ and -0.006 , respectively, and $T=500$; Figure 5), decreasing as the serial correlation spread, $\phi-\rho$ or $d-\rho$, or the sample size $T$, increase, independent of the (inverse) signal to noise ratio.

Differently, the downward bias in $\phi$ is increasing with the degree of persistence of the common factor $d$, the (inverse) signal to noise ratio $s / n^{-1}$, and the serial correlation spread, $\phi-\rho$ or $d-\rho$, yet decreasing with the sample size $T$ (Figures 1 and 3 ).

For the non integrated case (Figure 1), there are only few cases $(\phi-$ $\rho=0.4,0.6,0.8)$ when a $10 \%$, or larger, bias in $\phi$ is found, occurring when the series are particularly noisy $\left(s / n^{-1}=4\right)$; for the stationary long memory case a $10 \%$ bias, or smaller, is found for $s / n^{-1} \geq 2$, while for the non stationary

\footnotetext{
${ }^{11} \mathrm{~A}$ full set of results is available in the working paper version of this paper or upon request from the author.
} 
long memory case for $s / n^{-1} \geq 1$ and a (relatively) large sample $(T=500)$ (Figure 3). Increasing the cross-sectional dimension $N$ yields improvements (see the next section).

Also very satisfactory is the estimation of the unobserved common stochastic factor, as the $I C$ statistic is always below $0.2(0.14(0.10)$, on average, for $T=100(T=500)$ for the non integrated case; $0.06(0.03)$, on average, for $T=100(T=500)$ for the integrated case). Moreover, the correlation coefficient between the actual and estimated common factors is always very high, 0.98 and 0.99, on average, respectively, for both sample sizes (Figures 2 and 4$)$.

Results for smaller and larger cross-sectional samples In Figures 1-2 and 3-4 (center plots) the bias for the $\phi$ parameter and the correlation coefficient between the actual and estimated common factors are also plotted, for different cross-sectional dimensions, i.e., $N=5,10,15,50$, for the non integrated and integrated cases, respectively; statistics for the $\rho$ parameter are not reported, as the latter is always unbiasedly estimated, independently of the cross-sectional dimension.

As is shown in the plots, the performance of the estimator crucially depends on $T, N$, and $s / n^{-1}$.

For the non integrated case (Figure 1), when the (inverse) signal to noise ratio is low, i.e., $s / n^{-1} \leq 0.5$, the downward bias is already mitigated by using a cross-sectional sample size as small as $N=5$, for the case of $T=100$ observations; as $N$ increases, similar results are obtained for higher $s / n^{-1}$, i.e., $N=10,15$ and $s / n^{-1} \leq 1$, or $N=50$ and $s / n^{-1} \leq 4$. For a larger sample size, i.e., $T=500$, similar conclusions hold, albeit, for the $N=5$ case, the (inverse) signal to noise ratio can be higher, i.e., $s / n^{-1} \leq 1$; similarly for the $N=10,15$ case with $s / n^{-1} \leq 2$.

For the integrated case (Figure 3) conditions are slightly more restrictive; in particular, for the stationary long memory case, when the (inverse) signal to noise ratio is low, i.e., $s / n^{-1} \leq 0.5$, the downward bias is already mitigated by setting $N=5$ and $T=100$; similar results are obtained for higher $s / n^{-1}$ and $N$, i.e., $N=10,15$ and $s / n^{-1} \leq 1,2$, or $N=50$ and $s / n^{-1} \leq 4$. Similar conclusions can be drawn for $T=500$, albeit, holding $N$ constant, accurate estimation is obtained also for higher $s / n^{-1}$. Similarly also for the non stationary case (long memory or I(1)); yet, holding $T$ constant, either larger $N$, or lower $s / n^{-1}$, would be required for accurate estimation.

The above findings are corroborated by the estimated correlation coefficients between the actual and estimated common factors (Figures 2 and 4), showing that satisfactory estimation (a correlation coefficient higher than 
0.9) can be obtained also in the case of a small temporal sample size, provided the (inverse) signal to noise ratio is not too high, and/or the cross-sectional dimension is not too low $\left(s / n^{-1} \leq 1\right.$ and $N=5 ; s / n^{-1} \leq 2$ and $N=10$; $s / n^{-1} \leq 4$ and $\left.N=15\right)$.

\subsubsection{The structural change case}

While concerning the estimation of the $\rho$ parameter no sizable differences can be found for the designs with structural change, relatively to the case of structural stability ${ }^{12}$, the complexity of the break process may on the other hand affect estimation accuracy for the $\phi$ parameter, worsening as the number of break points increases, particularly when the temporal sample size is small $(T=100)$.

Yet, for the no integration case (Figure 1), already for $T=500$, the performance is very satisfactory for both designs, independently of the (inverse) signal to noise ratio $s / n^{-1}$; differently, for $T=100$ the performance is satisfactory (at most a $10 \%$ bias) only when the series are not too noisy $\left(s / n^{-1} \leq 1\right)$. Also, similar to the structural stability case, the (downward) bias in the $\phi$ parameter is increasing with the degree of persistence of the common factor $d$, the (inverse) signal to noise ratio $s / n^{-1}$, and $\phi-\rho$ or $d-$ $\rho$, yet decreasing with the sample size $T$.

Coherent with the above results, satisfactory estimation of the unobserved common stochastic factor (Figure 2) and break process can also be noted (Figure 5, columns 1-3); for the common stochastic factor, the $I C$ statistic is in fact always below 0.2 for the $T=500$ case $(0.11$ and 0.13 , on average, for the single break point and two-break points case, respectively) and below 0.3 for the $T=100$ case ( 0.17 and 0.20 , on average), while the actual and estimated common factors are strongly correlated: for $T=100(T=500)$, on average, the correlation coefficient is 0.96 (0.98) for the single break point case and 0.93 (0.97) for the two-break points case. Very accurate is also the estimation of the common break process (the $I C$ statistic is never larger than 0.15 for $T=100$ and 0.075 for $T=500$ ).

Concerning the integrated case, some differences relatively to the non integrated case can be noted; as shown in Figure 5 (columns 2-4), albeit the recovery of the common break process is always very satisfactory across the various designs, independently of the sample size (the $I C$ statistic is never larger than 0.14), performance slightly worsens, as the complexity of the

\footnotetext{
${ }^{12}$ The average bias is -0.04 and -0.01 , independent of the break process design and integration properties, when $T=100$ and $T=500$, respectively. Moreover, similar to the structural stability case the bias is decreasing as $\phi-\rho, d-\rho$, or the sample size $T$ increase, independent of the (inverse) signal to noise ratio.
} 
break process and persistence intensity $(d)$ increase: the average correlation coefficient between the estimated and actual break processes falls from 1 when $d=0.2$ (single break point case) to 0.93 when $d=1$ (two-break point case).

Moreover, concerning the estimation of the common stochastic factor (Figure 4 ), for the covariance stationary case $(d<0.5)$ results are very close to the non integrated case, i.e., an $I C$ statistic (not reported) always below 0.2 for the $T=500$ case $(0.12$ and 0.14 , on average, for the single break point and two-break points case, respectively) and below 0.3 for the $T=100$ case ( 0.21 and 0.24 , on average, respectively); the correlation coefficient is also very high (0.94 and 0.91, on average, $T=100 ; 0.97$ and 0.96 , on average, $T=500)$.

Differently, for the non stationary case performance is worse, showing average $I C$ statistics (not reported) of $0.32(0.32)$ and $0.42(0.44)$, respectively, for the single and two-break points case and $T=100(T=500)$; the average correlation coefficient is $0.79(0.78)$ and 0.68 (0.66), respectively.

Consistent with the above results is also the worsening in the estimation of the common factor autoregressive parameter $\phi$, for the $d=0.8$ and $d=1$ case, while comparable results to the short memory case can be found for $d<0.5$.

\section{LIBOR-OIS spreads: empirical properties and information content}

The recent turbulence in money, credit and financial markets has raised some questions about the "controllability" by central banks of the term structure of interest rates. In fact, while central banks have generally kept close control of very-short term unsecured money market rates (i.e., for overnight interbank deposits) and were also able to keep a steady influence on some longer-term money market interest rates (i.e., overnight index swap rates and general collateral repo rates), they seemed at pain to steer the evolution of the term structure of unsecured money market rates (i.e., LIBOR rates), particularly in the early stages of the subprime crisis.

The role of liquidity and counterparty (credit) risks, in explaining money market spreads dynamics and their term structure, is a much debated issue in this respect. On the one side of the debate the subprime crisis has been seen as one of banking solvency (Taylor and Williams, 2009; Afonso et al., 2011); hence, liquidity interventions by the Fed during the crisis have been criticized for being either wrong or misguided and, at best, having had no effect. 
On the other side of the debate the crisis has been seen as evolving through various stages, being the initial stage marked mainly by liquidity problems, that subsequently "metastasized" into a solvency crisis; in this view, Fed liquidity injections have been seen as rather appropriate and successful, at least during the first stages of the turbulence (see Christensen, 2009; Christensen et al., 2009; Armantier et al., 2008; Wu, 2011; Frank and Hesse, 2009).

The paper contributes to the debate by assessing the empirical properties of term structure of US LIBOR-OIS spreads (OIS spreads, thereafter $)^{13}$, over the period May 62002 through August 3 2012. One- and two-week and one- through twelve-month maturities, for a total of 14 time series and 2675 working days, are considered. The data source is REUTERS. The time span investigated allows to gauge insights on risk dynamics not only during the early stages of the subprime crisis, but also over its post-crisis evolution, as well as during the recent euro area sovereign debt crisis. As both LIBOR and OIS rates incorporate expectations of the average overnight rate until maturity, the latter cancel out when computing OIS spreads using rates of the same maturity. Then, if the resulting spreads are positive it is likely that this is due to counterparty risk, which is priced in the LIBOR rate but not in the OIS rate. Nevertheless, the spread is also likely to reflect liquidity funding/hoarding risk, as well as the state of investors confidence. ${ }^{14}$ Overall, LIBOR-OIS spreads can be seen as indicators of banks'assessment of the creditworthiness of other financial institutions and liquidity conditions, and more generally as a measure of stress conditions in the interbank market.

As is shown by the empirical results, accurate modeling of the persistence properties of US OIS spreads and the understanding of the effects of the recent financial crises do require a comprehensive strategy, actually made available by the proposed FI-HF-VAR model.

\footnotetext{
${ }^{13}$ LIBOR is the acronym for London interbank offered rate; LIBOR rates are the floating rates of interest that banks apply to lend money to each other at various maturities. OIS is the acronym for Overnight Index Swap; OIS rates are the fixed rates of swaps contracts for various maturities, whereby one party to the contract pays the fixed rate and in exchange receives the average overnight interest rate over the maturity of the contract. US OIS spreads are then based on LIBOR Eurodollar rates and OIS rates derived from the Federal Reserve's Fed Funds rate.

${ }^{14}$ If a bank has a rating downgrade its credit lines are tightened as a result, exposing it to higher financing risk; moreover, faced with larger uncertainty about the valuation of its own assets and the availability of longer-term funding, a banks would also be led to build up "excess reserves" (Allen et al., 2009; Caballero and Krishnamurthy, 2008). Moreover, a higher spread (high LIBOR) might signal decreased willingness to lend by major banks, while a lower spread might signal a more liquid interbank market.
} 


\subsection{Testing for structural breaks}

The investigation of the persistence properties of the OIS spreads term structure is based on testing for structural breaks and long memory.

The structural break analysis is performed using the Bai and Perron (1998) $U D_{\max }$ test, implemented on OIS spreads data $\left(x_{i, t}: x_{t}^{1 w}, \ldots, x_{t}^{12 m}\right)$ sampled at different frequency; firstly, structural break tests are carried out using calendar month OIS level observations and the number and location of breaks determined also by means of information criteria (BIC, LWZ); this implies that no regimes lasting less then twenty/twenty-three working days are estimated. Then, in order to refine the estimated breaks location, the $U D_{\max }$ test is performed using daily data, within a range of data centered about the break-point determined by the monthly data analysis. The results of the structural break analysis are reported in Table 1.

As shown in Table 1 (Panel A and B), while breaks are similarly located along the OIS spreads term structure, some differences are found between levels and volatilities and the short- and long-end of the term structure concerning their number. In fact, three break points can be detected for the OIS spreads series in level at the short-end of the term structure, i.e., for the 1-week, 2-week and 1-month maturities, while five break points can be detected for all the other maturities. Moreover, two breaks in volatility are found for all the maturities.

Concerning OIS spreads level series (Table 1, Panel A), the first break point may be located between August 9 and 14 2007, depending on maturity: the money market stress which set in since August 8 2007, following the BNP Paribas episode, was indeed sizable, as the average spread moved from a range of 7b.p.-13b.p. to a range of 40b.p.-78b.p. until September 152008. The crisis triggered interventions by the European Central Bank and the US Federal Reserve, injecting overnight funds of $€ 95$ billions and US $\$ 38$ billions, respectively, on August 9 and August 10 2007. Additional measures were taken by the ECB, the US Federal Reserve and other central banks in the following days. ${ }^{15}$

Moreover, the second break point may be located between September 16 and 19 2008, according to maturity: since Lehman Brothers bankruptcy (September 15 2008) OIS spreads climbed rapidly, to reach maximum values in the range of 272b.p. to 354b.p. between October 8 and October 13 2008, according to maturity (144b.p. to 230b.p. on average, over the period September through December 2008). In the face of major difficulties in the banking sector in the US and Europe, various forms of liquidity injection

\footnotetext{
${ }^{15}$ See Brunnermeier (2009) and Acharya and Richardson (2009) for an assessment of the US subprime crisis.
} 
and unconventional monetary policy measures were taken by central banks, aiming at defreezing the interbank and credit markets, and easing the banking sector from the burden of unperforming loans, as well as to facilitate its recapitalization, supported by governments interventions. ${ }^{16}$

Starting from mid-October 2008, spreads have progressively narrowed, albeit with different speed across maturities, i.e., at a quicker pace for shorter than longer maturities. In particular, the location of the third break point can be set between December 9 and 122008 for the one-week, two-week and 1-month rates, and on December 172008 for the remaining maturities. For the former maturities average OIS spreads levels, similar to those prevailing before the subprime crisis, can be found since January 2009 through the end of the sample (August 3 2012); for longer maturities only a sizable contraction in the average OIS spreads levels can be noted, as they have kept fluctuating about much higher values than those prevailing before the crisis.

Finally, the post-subprime crisis period is marked by two additional break points at the medium- long-end of the OIS spreads term structure. The former, i.e., the fourth break point, is located between August 24 and September 11 2009, according to maturity, following the end of the US recession (June 2009, according to the NBER's Business Cycle Dating Committee): the contraction in OIS spreads levels after August/September 2009 is sizable for maturities beyond 1-month, i.e., from 9b.p.-85b.p. (December 92008 through August 21 2009) to 8b.p.-63b.p. (August 242009 through September 5 2011). The latter, i.e., the fifth break point, is located between September 6 and 12 2011, according to maturity, corresponding to the spillover of the euro area sovereign debt crisis to Italy; since September 2011 OIS spreads levels have sizably increased, i.e., 8b.p.-90b.p. on average, climbing up to 19b.p.-91b.p. (2-month to 1-year maturities) on June 1 2012; some reversion to lower values can be noted thereafter (up to August 3 2012, the last day in our sample). ${ }^{17}$

\footnotetext{
${ }^{16}$ See Veronesi and Zingales (2009) and Bianco (2012) for a summary of government measures in support of the US banking system. See Krishnamurthy and Vissing-Jorgensen (2011) and D'Amico et al. (2012) for an account of the effects of the quantitative easing policy implemented by the Fed in 2008-2009 and 2010-2011. See also Reis (2009).

${ }^{17}$ Some relevant events along the EMU sovereign debt crisis time-line are as follows: April 11 2010, when EMU leaders agreed on a $€ 30$ billion bailout plan for Greece; April 27 2010, when S\&P dowgraded Greece debt below investment rating and Portugues debt two notches, also issuing a negative outlook; April 28 2010, when S\&P downgraded Spain debt to AA-; May 8 2010, when EMU leaders agreed on a $€ 100$ billion bailout plan for Greece; November 22 2010, when Ireland accepted the EMU-IMF bailout package; September 19 2011, when S\&P downgraded Italy's public debt one notch from A to A-, October 13 2011, when S\&P downgraded Spain's public debt one notch from AA to AA-, November 25 2011, when S\&P downgraded Belgium's public debt one notch from AA+ to AA, January 13 2012, when S\&P downgraded Italy's public debt two notches to BBB+, as
} 
Differently, two break points can be detected for the volatility proxy, computed as absolute changes in the OIS spreads levels, i.e., $\sigma_{i, t}=\left|\Delta x_{i, t}\right|{ }^{18}$ As shown in Table 1 (Panel B), the former is located between July 252007 and August 22007 and the latter between December 222008 and February 11 2009, marking therefore three volatility regimes, similarly to what found for the short-end of the OIS spread levels term structure: the pre-subprime crisis period (May 62002 through August 8 2007); the subprime crisis period (August 92007 through December 23 2008) ${ }^{19}$, which may be related to the two waves of money market stress triggered by the BNP Paribas episode (August 82007 through September 15 2008, the former) and Lehman Brothers bankruptcy (September 162008 through December 23 2008, the latter); the post-subprime crisis period (December 242008 through August 3 2012), associated with the subprime crisis resolution and the successive euro area sovereign debt crisis, which, while started already in February 2010 with the negative assessment by the EU-IMF of Greece's public finances, spread to Portugal, Spain and Ireland by November 2010, to eventually spill over to Italy by September 2011.

A sizable increase on average volatility can be noted over the subprime crisis period relatively to the pre-crisis period, i.e., from a range 1b.p.-4b.p. to 5b.p.-8b.p.; a similarly sizable contraction can be noted over the postsubprime crisis period, as average volatility dampened to values even smaller than the pre-crisis period, in the range 0.5b.p.-1.4 b.p.

\subsubsection{Estimating the structural break process}

Candidate break processes are estimated by means of an OLS regression of each OIS spread level $\left(x_{i, t}: x_{t}^{1 w}, \ldots, x_{t}^{12 m}\right)$ on dummies $\left(D_{m, j}, j=1, . ., 5\right)$ computed according to the findings of the structural break analysis; the regression functions are then specified as follows

$$
\begin{aligned}
x_{i, t} & =b_{i, t}+e_{i, t} \quad i=1, \ldots, 14 \\
b_{i, t} & =\alpha_{i, 0}+\sum_{j=1}^{k} \alpha_{i, j} D_{m, j, t}+\sum_{j=1}^{k} \gamma_{i, j}\left(D_{m, j, t} * T_{t}\right),
\end{aligned}
$$

well public debt for France, Austria, Spain and other five euro area members, maintaining AAA rating only for Finland, Germany, Luxembourg and the Netherlands. See De Santis (2012) for an account of the euro area sovereign debt crisis.

${ }^{18}$ Monthly figures are obtained by means of the realized volatility estimator, computed using calendar month daily observations.

${ }^{19}$ For the OIS spreads level series the end of the subprime crisis regime is selected between December 9 and 17 2008, according to maturity. 
where $k=3,5$, according to maturity. In particular, $D_{m, 1}$ is a (first financial stress wave) step dummy variable with unity value over the period August 9/14 2007 through August 32012 inclusive, $D_{m, 2}$ is a (second financial stress wave) step dummy variable with unity value over the period September 16/19 2008 through August 32012 inclusive, $D_{m, 3}$ is a (first financial stress resolution) step dummy variable with unity value over the period December 9/18 2008 through August 32012 inclusive, $D_{m, 4}$ is a (second financial/economic stress resolution) step dummy variable with unity value over the period $\mathrm{Au}$ gust 24/September 112009 through August 32012 inclusive, $D_{m, 5}$ is a (euro area crisis) step dummy variable with unity value over the period September 6/12 2011 through August 32012 inclusive. The above dummies have also been interacted with a linear time trend $\left(T_{t}=1,2, \ldots, 2675\right)$.

Similar regressions are performed using volatility proxies

$$
\begin{aligned}
\left|\Delta x_{i, t}\right| & =c_{i, t}+u_{i, t} \quad i=1, \ldots, 14 \\
c_{i, t} & =\beta_{i, 0}+\sum_{j=1}^{q} \beta_{i, j} D_{v, j, t},
\end{aligned}
$$

where $q=2$ for all the OIS spreads maturities. For the latter case $D_{v, 1}$ is a (financial stress wave) step dummy variable with unity value over the period July 25/August 22007 to August 32012 inclusive, $D_{v, 2}$ is a (financial stress resolution) step dummy variable with unity value over the period December 22 2008/February 92009 to August 32012 inclusive.

An exponential smoother is applied to the estimated break processes $\hat{b}_{i, t}$ and $\hat{c}_{i, t}$, in order to yield smooth transition across regimes; the smoothing parameter $p$ in

$$
k_{s, i, t}=p s_{i, t-1}+(1-p) k_{i, t}
$$

where $k_{i, t}=\hat{b}_{i, t}, \hat{c}_{i, t}$ is the generic break process to be smoothed, $t=1, \ldots, T$, is selected in order to best fit $\left(R^{2}\right)$ the transition across regimes to actual data. This yields $p=0.69$ for the OIS spreads level series and $p=0.51$ for the OIS spreads volatility series. Validation of the estimated candidate break processes is performed by assessing the long memory properties of the corresponding break-free series (see next section).

\subsection{Long memory analysis}

Due to structural change in mean and variance, normalized break-free OIS spreads series are computed, i.e., $\hat{l}_{i, t}=\frac{x_{i, t}-\hat{b}_{s, i, t}}{\hat{\sigma}_{i, t}}$, where $\hat{\sigma}_{i, t}$ is the estimated 
unconditional standard deviation for the break-free series over the three selected volatility regimes, i.e., $\hat{\sigma}_{i, t}=\hat{\sigma}_{1}$ over the period May 62002 through July 25/August 2 2007; $\hat{\sigma}_{i, t}=\hat{\sigma}_{2}$ over the period July 26/August 32007 through December 22 2008/February $92009 ; \hat{\sigma}_{i, t}=\hat{\sigma}_{3}$ over the period December 23 2008/February 102009 through August 32012.

Long memory analysis is then performed for both the series in levels $\left(x_{i, t}\right)$ and break-free $\left(\hat{l}_{i, t}\right)$, using the broad band log-periodogram estimator (BBLP) of the fractional differencing parameter proposed by Moulines and Soulier (1999); additional testing against spurious long memory is performed by means of the Dolado et al. (1995) augmented Dickey-Fuller test (DGM) and the Shimotsu (2006) KPSS test (SKPSS), both modified to account for a non linear (smooth) break process in computing critical values, and the LM-test proposed by Demetrescu et al. (2006) (LM). A similar analysis is carried out for the volatility proxies, both in levels $\left(\left|\Delta x_{i, t}\right|\right)$ and break-free $\left(\hat{v}_{i, t}=\left|\Delta x_{i, t}\right|-\hat{c}_{s, i, t}\right)$.

As shown in Table 2 (columns 1 and 2), strong persistence can be found for the OIS spreads series, both in levels and break-free (Panel A); the estimated fractional differencing parameter is in the range 0.85 to 1.10 and 0.46 to 0.69 , for the series in levels and break-free, respectively. A statistically significant hump-shaped profile can be noted in the cross-section of persistence, the latter increasing with maturity up to the two-month horizon and decreasing thereafter.

A joint test for the null hypothesis of equal fractional differencing parameter across the OIS spreads term structure, i.e., $H_{0}: d_{1}=d_{2}=\ldots=d_{n}=\bar{d}$, $n=14$, versus $H_{1}: H_{0}$ is incorrect, where $\bar{d}$ is the mean value of the estimates of the fractional differencing parameter across series, can be carried out using the Wald test statistics

$$
\hat{W}_{f}=(T \hat{d})^{-1}\left(T \Lambda T^{\prime}\right)^{-1}(T \hat{d}),
$$

similarly to Ohanissian et al. (2008), where $\hat{d}=\left(\begin{array}{llll}\hat{d}_{1} & \hat{d}_{2} & \cdots & \hat{d}_{n}\end{array}\right)^{\prime}$ is the multivariate BBLP estimator, $\Lambda=\operatorname{diag}\left(\begin{array}{cccc}\sigma_{\hat{d}_{1}}^{2} & \sigma_{\hat{d}_{2}}^{2} & \cdots & \sigma_{\hat{d}_{n}}^{2}\end{array}\right)$ its asymptotic variance-covariance matrix,

$$
\underset{(n-1, n)}{T}=\left(\begin{array}{cccccc}
1-\frac{1}{n} & -\frac{1}{n} & -\frac{1}{n} & \cdots & -\frac{1}{n} & -\frac{1}{n} \\
-\frac{1}{n} & 1-\frac{1}{n} & -\frac{1}{n} & \cdots & -\frac{1}{n} & -\frac{1}{n} \\
-\frac{1}{n} & -\frac{1}{n} & 1-\frac{1}{n} & \cdots & -\frac{1}{n} & -\frac{1}{n} \\
\cdots & \cdots & \cdots & \cdots & \cdots & \cdots \\
-\frac{1}{n} & -\frac{1}{n} & -\frac{1}{n} & \cdots & 1-\frac{1}{n} & -\frac{1}{n}
\end{array}\right),
$$

which has an asymptotic $\chi_{(n-1)}^{2}$ distribution under the null hypothesis. As 
shown in Table 3 (columns 1 and 2; last row), the test yields values of 37.2 and 56.4 for the series in levels and break-free, respectively, pointing to rejection of the null hypothesis of homogeneous persistence across the OIS spreads term structure.

Implementation of the DGM and DKH tests requires the estimation of the following OLS regression

$$
y_{i, t}=\phi_{i} y_{i, t-1}^{*}+\sum_{j=1}^{p} a_{i, j} y_{i, t-j}+\varepsilon_{i, t} \quad t=1, \ldots, T,
$$

where $y_{i, t}=(1-L)^{\hat{d}_{i}} \hat{l}_{i, t}, i=1, \ldots, 14, y_{i, t-1}^{*}=\hat{l}_{i, t-1}$ for the DGM test and $y_{i, t-1}^{*}=\sum_{j=1}^{t-1} \frac{y_{t-j}}{j}$ for the DKH test. The null hypothesis of long memory persistence of degree $\hat{d}_{i}$, against the alternative of $\mathrm{I}(0)$ plus smooth non linear break process type of persistence (DGM) or different degree of long memory persistence $(\mathrm{DKH})$, is $H_{0}: \phi_{i}=0$ against $H_{1}: \phi_{i} \neq 0$, and is tested using the t-ratio test statistics

$$
t_{\phi_{i}}=\hat{\phi}_{i} / \hat{\sigma}_{\hat{\phi}_{i}}
$$

which, for the DGM test, has a non standard asymptotic distribution in the nonstationary long memory case and a $N(0,1)$ distribution for the stationary long memory case; a $N(0,1)$ distribution, for both the stationary and nonstationary long memory case, for the DKH test. The order of the augmentation term is selected according to the deterministic rule of Schwert (1989), i.e., by setting $p=4 *(T / 100)^{1 / 4}$. Critical values for the DGM test are computed through Monte Carlo simulation in order to match the features of the estimated candidate break processes.

The modified Shimotsu (2006) KPSS statistic is finally defined as

$$
\eta_{i, n l b p}=T^{-2} \sum_{t=1}^{T} S_{i, t}^{2} / s_{i, p}^{2}
$$

where $S_{i, t}=\sum_{k=1}^{t} y_{i, k}, s_{i, p}^{2}=\frac{1}{T} \sum_{t=1}^{T} y_{i, t}^{2}+\frac{2}{T} \sum_{q=1}^{p}\left(1-\frac{q}{p+1}\right) \sum_{t=q+1}^{T} y_{i, t} y_{i, t-q}$, and $y_{i, t}=(1-L)^{\hat{d}_{i}} \hat{l}_{i, t}$. The null hypothesis of long memory persistence of degree $\hat{d}_{i}$, against the alternative of $\mathrm{I}(0)$ plus smooth non linear break process type of persistence, is then tested. The distribution of the test statistics under the null is non standard and therefore critical values are obtained through Monte Carlo simulation. 
As shown in Table 2 (Panel A), the null of long memory is never rejected at the $1 \%$ level by the DKH and DGM tests, while the null of I(0) plus non linear break process is always rejected by the SKPSS test at the $1 \%$ level (apart from the 1-week and 2-week maturities (5\% level). The results of the tests are then coherent with the findings of long memory for the breakfree series, also validating the estimated break processes, as antipersistence $\left(-0.5<\hat{d}_{i}<0\right)$ is induced by the removal of a spurious break process (Granger and Hyung, 2004).

Evidence of long memory can also be detected for the volatility series, both in levels and break-free; the estimated fractional differencing parameter is in the range 0.31 to 0.41 and 0.16 to 0.26 , for the series in levels and breakfree, respectively (Table 2, Panel B). Differently from what found for the OIS spreads levels, evidence of common persistence across the term structure can be found as well ( $\hat{W}_{f}$ statistics; last row in Table 2$)$, with average estimated values for the fractional differencing parameters equal to 0.35 and 0.19 , respectively. Evidence of genuine long memory in volatility is also confirmed by the results of the SKPSS, DGM and DKH tests.

\subsubsection{Fractional differencing parameter constancy tests}

Persistence stability over time has been investigated by splitting the sample according to two different scenarios. The first scenario assumes a permanent break in persistence occurring after August 8 2007; the second scenario, in addition to the previous break, allows for a second change in persistence occurring after December 8 2008. The two scenarios are then consistent with the structural break analysis carried out using the OIS spreads series in levels.

The null hypothesis is therefore $H_{0}: d_{i, 1}=d_{i, 2}=\bar{d}_{i}, i=1, \ldots, 14$, versus $H_{1}: H_{0}$ is incorrect, for the first scenario and $H_{0}: d_{i, 1}=d_{i, 2}=d_{i, 3}=\bar{d}_{i}, i=$ $1, \ldots, 14$, versus $H_{1}: H_{0}$ is incorrect, for the second scenario. In both cases $\bar{d}_{i}$ is the mean value of the estimates of the fractional differencing parameter across regimes. The above test can be carried out considering each series at the time and all series jointly. In the first case, when the analysis is performed for each series at the time, the Wald test statistic in (33) has elements defined as

$$
\hat{d}=\left(\begin{array}{ll}
\hat{d}_{i, 1} & \hat{d}_{i, 2}
\end{array}\right)^{\prime}, \Lambda=\operatorname{diag}\left(\begin{array}{cc}
\sigma_{\hat{d}_{i, 1}}^{2} & \sigma_{\hat{d}_{i, 2}}^{2}
\end{array}\right) \text { and } \underset{(1,2)}{T}=\left(\begin{array}{cc}
1-\frac{1}{2} & -\frac{1}{2}
\end{array}\right) \text { for }
$$

the first scenario and

$$
\hat{d}=\left(\begin{array}{ccc}
\hat{d}_{i, 1} & \hat{d}_{i, 2} & \hat{d}_{i, 3}
\end{array}\right)^{\prime}, \Lambda=\operatorname{diag}\left(\begin{array}{ccc}
\sigma_{\hat{d}_{i, 1}}^{2} & \sigma_{\hat{d}_{i, 2}}^{2} & \sigma_{\hat{d}_{i, 3}}^{2}
\end{array}\right) \text { and } \underset{(2,3)}{T}=\left(\begin{array}{ccc}
1-\frac{1}{3} & -\frac{1}{3} & -\frac{1}{3} \\
-\frac{1}{3} & 1-\frac{1}{3} & -\frac{1}{3}
\end{array}\right)
$$

for the second scenario. This yields the Wald test statistic $\hat{W}_{i, 2}$ and $\hat{W}_{i, 3}$, $i=1, \ldots, 14$, for the first and second scenario, respectively.

In the second case, when the test is performed jointly for all series ( $n=$ 
14), the elements of the Wald test statistics in (33) are defined as $\hat{d}=$

$$
\begin{aligned}
&\left(\begin{array}{lllllllll}
\hat{d}_{1,1} & \hat{d}_{1,2} & \cdots & \hat{d}_{n, 1} & \hat{d}_{n, 2}
\end{array}\right)^{\prime}, \Lambda=\operatorname{diag}\left(\begin{array}{ccccccc}
\sigma_{\hat{d}_{1,1}}^{2} & \sigma_{\hat{d}_{1,2}}^{2} & \cdots & \sigma_{\hat{d}_{n, 1}}^{2} & \sigma_{\hat{d}_{n, 2}}^{2}
\end{array}\right) \text { and } \\
& \underset{(2(n-1), 2 n)}{T}=\left(\begin{array}{cccccccc}
1-\frac{1}{2} & -\frac{1}{2} & 0 & \cdots & 0 & \cdots & 0 & 0 \\
0 & 0 & 1-\frac{1}{2} & -\frac{1}{2} & 0 & \cdots & 0 & 0 \\
\cdots & \cdots & \cdots & \cdots & \cdots & \cdots & \cdots & \\
0 & 0 & 0 & \cdots & 0 & \cdots & 1-\frac{1}{2} & -\frac{1}{2}
\end{array}\right)
\end{aligned}
$$

for the first scenario, and $\hat{d}=\left(\begin{array}{lllllll}\hat{d}_{1,1} & \hat{d}_{1,2} & \hat{d}_{1,3} & \cdots & \hat{d}_{n, 1} & \hat{d}_{n, 2} & \hat{d}_{n, 3}\end{array}\right)^{\prime}, \Lambda=$ $\operatorname{diag}\left(\begin{array}{ccccccc}\sigma_{\hat{d}_{1,1}}^{2} & \sigma_{\hat{d}_{1,2}}^{2} & \sigma_{\hat{d}_{1,3}}^{2} & \cdots & \sigma_{\hat{d}_{n, 1}}^{2} & \sigma_{\hat{d}_{n, 2}}^{2} & \sigma_{\hat{d}_{n, 3}}^{2}\end{array}\right)$ and

$$
\underset{(3(n-1), 3 n)}{T}=\left(\begin{array}{ccccccccccc}
1-\frac{1}{3} & -\frac{1}{3} & -\frac{1}{3} & 0 & \cdots & 0 & 0 & \cdots & 0 & 0 & 0 \\
0 & 0 & 0 & 1-\frac{1}{3} & -\frac{1}{3} & -\frac{1}{3} & 0 & \cdots & 0 & 0 & 0 \\
\cdots & \cdots & \cdots & \cdots & \cdots & \cdots & \cdots & \cdots & \cdots & \cdots & \cdots \\
0 & 0 & 0 & 0 & \cdots & 0 & 0 & \cdots & 1-\frac{1}{3} & -\frac{1}{3} & -\frac{1}{3}
\end{array}\right)
$$

for the second scenario. This yields the Wald test statistics $\hat{W}_{j, 2}$ and $\hat{W}_{j, 3}$ for the first and second scenario, respectively.

The Wald test in (33) is also employed to test for the null hypothesis of equality of the fractional differencing parameter across the OIS spreads term structure for each of the subsamples determined according to the above scenarios. This yields the Wald test statistics $\hat{W}_{\text {pre }}$ and $\hat{W}_{c / p}$ for the pre-crisis and crisis/post crisis subsamples, respectively, for the first scenario; $\hat{W}_{\text {pre }}, \hat{W}_{c}$ and $\hat{W}_{\text {post }}$ for the pre-crisis, crisis and post-crisis subsamples, respectively, for the second scenario.

As shown in Table 3, there is strong evidence of temporal instability in the fractional differencing parameter for each of the OIS spreads and both scenarios. The null of temporal stability is always rejected at the $5 \%$ level, apart from the 2-month maturity, for the first scenario, while it is rejected for any maturity for the second scenario (column $5, \hat{W}_{i, 2} ;$ column $6, \hat{W}_{i, 3}$ ). Coherently, the null hypothesis of temporal stability is also strongly rejected when assessed jointly for all series $\left(\hat{W}_{j, 2}\right.$ and $\hat{W}_{j, 3}$; last row in Table 3$)$.

As a consequence of the subprime crisis, a sizable increase in persistence can then be detected along the whole OIS spreads term structure, from stationary long memory for the pre-crisis period (apart from the 2-week through 5 -month maturities), to non stationary long memory for the crisis and post crisis periods.

Moreover, while the null of equal fractional differencing parameter across the term structure is rejected for the pre-crisis period $\left(\hat{W}_{\text {pre }}\right.$, column 1 , last row), the latter is largely not rejected for the crisis and post-crisis periods $\left(\hat{W}_{c}\right.$ 
and $\hat{W}_{\text {post }}$; columns 6 and 7 , last row). Average estimates of the fractional differencing parameter are 0.47 and 0.72 for the pre-crisis and crisis/postcrisis periods, respectively, for the first scenario; $0.47,0.55$ and 0.93 for the pre-crisis, crisis and post-crisis periods, respectively, for the second scenario.

To assess the robustness of the findings, the LM fractional differencing parameter constancy test of Hassler and Meller (2008) is also implemented. The test is related to the LM test of Demetrescu et al. (2006) and requires the estimation of the following augmented OLS regression

$$
y_{i, t}=\phi_{i} y_{i, t-1}^{*}+\psi_{i} D_{t}(\lambda) y_{i, t-1}^{*}+\sum_{j=1}^{p} a_{i, j} y_{i, t-j}+\varepsilon_{i, t} \quad t=1, \ldots, T
$$

where $y_{i, t}=(1-L)^{\hat{d}_{i}} \hat{l}_{i, t}, i=1, \ldots, 14, y_{i, t-1}^{*}=\sum_{j=1}^{t-1} \frac{y_{t-j}}{j}$, and $D_{t}(\lambda)$ is a dummy variable taking a zero value over the subsample $t=1, \ldots,[\lambda T]$ and unity value over the subsample $t=[\lambda T]+1, \ldots, T$, with break fraction $\lambda$ varied over the interval $[\pi, 1-\pi]$ and $\pi \in(0,1 / 2)$. The null hypothesis of constant long memory persistence of degree $\hat{d}_{i}$, against the alternative of $\hat{d}_{i}+\psi_{i}$ persistence, is $H_{0}: \psi_{i}=0$ against $H_{1}: \psi_{i} \neq 0$, and is tested using the supremum of the sequence of squared t-ratio test statistics

$$
\sup _{\lambda \in[\pi, 1-\pi]} t_{\psi_{i}}^{2}(\lambda)
$$

where $t_{\psi_{i}}=\hat{\psi}_{i} / \hat{\sigma}_{\hat{\psi}_{i}}$. In the empirical implementation, following Hassler and Meller (2009), the order of the augmentation term is determined as $p=4 *(T / 100)^{1 / 4}$, while the break fraction is set to $\lambda=0.15$; critical values are tabulated using Monte Carlo simulation.

The results of the test reported in Table 3 are for the statistics $t_{\psi_{i}}^{2}(\lambda)$ computed with reference to the candidate break dates employed in the two above scenarios, i.e., August 92007 and December 8 2008, in addition to the two candidate break points selected by the Hassler and Meller (2009) test. As shown in the table, a change in persistence can be detected for the various maturities, taking place already in June 2007 for the 5- to 12-month maturities and in the aftermath of the subprime crisis (September 12 2007) for shorter maturities; the evidence is clear-cut (1\% significance level) for all the maturities, apart from the very short-end of the term structure. A second change in persistence ( $1 \%$ level) can also be detected, taking place after the selected end point for the crisis period (December 8 2008), i.e., in December 172008 for the 1- to 12-month maturities, and in February 72009 for the 1and 2-week maturities. 
Differently, as shown in Table 4, the null of common degree of persistence across the volatility term structure is never rejected at the $5 \%$ level $\left(\hat{W}_{\text {pre }}\right.$, $\hat{W}_{c / p}, \hat{W}_{c}$ and $\hat{W}_{\text {post }}$ statistics); similarly, no rejection of the null hypothesis of constant persistence across regimes is found for both scenarios, each maturity $\left(\hat{W}_{i, 2}, \hat{W}_{i, 3}\right)$ and for all maturities jointly $\left(\hat{W}_{j, 2}, \hat{W}_{j, 3}\right)$. The evidence of persistence constancy is also confirmed by the Hassler and Meller (2009) test, which does not allow to reject the null of stability of the fractional differencing parameter, at the $5 \%$ level, for any maturity.

\subsection{Testing for common deterministic and stochastic factors}

Principal components analysis is employed to assess the presence of common factors driving the term structure of OIS spreads levels and volatilities. The analysis is carried out for the estimated break processes $\left(\hat{b}_{s, i, t}: \hat{b}_{s, t}^{1 w}, \ldots, \hat{b}_{s, t}^{12 m}\right.$; $\left.\hat{c}_{s, i, t}: \hat{c}_{s, t}^{1 w}, \ldots, \hat{c}_{s, t}^{12 m}\right)$ and corresponding break-free series $\left(\hat{l}_{i, t}: \hat{l}_{t}^{1 w}, \ldots, \hat{l}_{t}^{1 y} ; \hat{v}_{i, t}\right.$ : $\left.\hat{v}_{t}^{1 w}, \ldots, \hat{v}_{t}^{12 m}\right)$, as well as for the OIS spreads levels and volatilities $\left(x_{i, t}: x_{t}^{1 w}, \ldots\right.$, $\left.x_{t}^{1 y} ;\left|\Delta x_{i, t}\right|:\left|\Delta x_{t}^{1 w}\right|, \ldots,\left|\Delta x_{t}^{12 m}\right|\right)$. Results are reported in Table 5.

As is shown in Table 5 (columns 1-3), the first principal component accounts for the bulk of total variance for the OIS spreads levels $\left(x_{i, t}, 95 \%\right.$; column 1) and for about $90 \%$, or over, of the variance for the 2-month and longer maturities; the second principal component accounts for a residual $4 \%$ of total variance, yet explaining a sizable proportion of the variance for each of the shortest maturities, i.e., about $40 \%$ for the 1-week, 2 -week and 1-month OIS spreads (column 2). Similar findings can be noted for the OIS spreads break process and break-free series $\left(\hat{b}_{s, i, t}\right.$, columns $3-4 ; \hat{l}_{i, t}$, columns 5-6). Moreover, the third principal component accounts for residual commonalities involving some of the maturities at the short-end of the term structure for the OIS spreads actual and break processes, while involving intermediate maturities for the break-free series.

Similar results can be noted for the OIS spreads volatility proxies, as the first three principal components account for about $100 \%$ of total variance for the actual volatility series $\left(\left|\Delta x_{i, t}\right|\right.$, columns 10-12) and their break-free components $\left(\hat{c}_{s, i, t}\right.$, columns 16-18); the latter evidence is consistent with the finding of three common long memory factors for the OIS spreads break-free series $\left(\hat{l}_{i, t}\right)$, which, according to ARCH tests, are strongly heteroskedastic (the p-value of the test is zero for each of the factors; not reported). In particular, for the actual volatility series, the first principal component accounts for about $76 \%$ of total variance and for about $65 \%$ to $90 \%$ of the variance for the 2-month and longer maturities; the second principal component accounts 
for a residual $15 \%$ of total variance and for about $50 \%$ of the variance for the shortest maturities; finally, the third principal component accounts for $5 \%$ of total variance and for about $20 \%$ of the variance for maturities within the 1- through 4-month interval; similar findings can be noted for the breakfree volatility series. Differently, $100 \%$ of total variance for the volatility break processes is accounted for by their first two principal components; the latter explain $96 \%$ and $4 \%$ of total variance, respectively; while the former component accounts for a proportion of variance in the range $70 \%$ to $99 \%$ for the volatility proxies, the second component accounts for up to $30 \%$ of the variance for maturities at the short-end of the volatility term structure.

According to the estimated loadings reported in Table 6, the extracted principal components bear the interpretation of level, slope and curvature factors for each subset of the series considered (levels and volatilities). In fact, in all cases the first factor is loaded on to each of the corresponding series with loading of the same sign, while opposite signs at the short- and long-end of the term structure can be noted for the loadings of the second principal component; moreover, similar signs at the short- and long-end of the term structure, yet opposite for intermediate maturities, can be noted for the loadings of the third principal component.

On the basis of the proposed interpretation and results, the first three principal components are selected for the break-free OIS spreads level and volatility series, while the first three principal components are selected for the OIS spreads break processes in mean and only the first two principal components for the OIS spreads break processes in volatility.

As shown in Figure 6, the first principal component estimated from the common break processes well tracks the overall level of the OIS spreads series, while the two additional components yield a correction mostly relevant at the short-end of the term structure. Moreover, consistent with the results of the long memory analysis, deviations from the overall level of the OIS spreads are indeed strongly persistent: full sample BBLP estimates of the fractional differencing parameter for the three (normalized) break-free OIS spreads common factors are in the range 0.46 to $0.60,0.53$ on average, and not statistically different at the $1 \%$ level. Differently, weaker persistence can be noted for the volatility factors, in the range 0.12 to $0.19,0.16$ on average.

\subsubsection{Testing for persistence stability in the common factors}

As for the break-free series, persistence stability tests are carried out considering two scenarios; the first scenario assumes a permanent break in persistence occurring after August 8 2007; the second scenario, in addition to the previous break, allows for a second change occurring after December 82008 . 
As shown in Table 7, consistent with the results yield by the univariate analysis, temporal instability in the degree of persistence can also be noted for the factors extracted from the OIS spread break-free level components, while constant persistence is found for the corresponding volatility factors; in particular, evidence of a strong increase in the persistence of the common stochastic factors (in mean) can be detected and associated with the effects of the subprime financial crisis. In fact, while stationary long memory, in the range 0.32 to $0.48,0.40$ on average, characterizes the pre-crisis sample, non-stationary long memory is found for the crisis/post-crisis (0.66 to 0.72$)$, crisis (0.55 to 0.76$)$ and post-crisis (0.73 to 0.87 ) periods. According to the $\hat{W}_{i, 2}$ and $\hat{W}_{i, 3}$ statistics, the increase in persistence is statistically significant at the $5 \%$ level for each factor, and at the $1 \%$ level for all the factors jointly ( $\hat{W}_{j, 2}$ and $\hat{W}_{j, 3}$ statistics), for both scenarios. Additional evidence of instability in the persistence parameter is provided by the Hasller and Meller (2009) test, pointing to statistically significant $t_{\psi_{i}}^{2}(\lambda)$ statistics (1\% level) also when computed with reference to the candidate break dates, i.e., August 92007 and December 8 2008; the optimally selected dates for the two break points are also located in the aftermath of the financial crisis (August 17-20 2007; October 5 2007) and few days earlier than Lehman Brothers bankruptcy (September 4-8, 2008).

In figure 7 , the results of BBLP moving window estimation of the persistence parameter for the three common factors in mean are presented. ${ }^{20}$ The increase in persistence in the aftermath of the trigger events for the two waves of money market stress, associated with the BNP Paribas (Agust 8 2007) and Lehman Brothers bankruptcy (September 15 2008), can clearly be noted in the plots; interestingly, while the BNP Paribas episode is associated with a swift increase in persistence, a smooth change followed the setting in of the crisis, as the Lehman Brothers episode might only be associated with an acceleration in its pace. On the basis of the overall findings, two break points in the persistence parameters are then selected for each common factor in mean, with break points located in August 92007 and September 16 2008.

\subsection{Estimation of the FI-HF-VAR model}

On the basis of the BIC information criterion, a sixth order diagonal VAR specification is selected for the $P(L)$ matrix in (2), with $D(L)$ matrix set according to the results of the long memory analysis; differently, a ninth order diagonal VAR specification is selected for the $C(L)$ matrix in (1). Moreover,

\footnotetext{
${ }^{20}$ Window size is set to 500 observations.
} 
the $i$ th generic element along the main diagonal of the conditional variancecovariance matrix $H_{t}$ in $(4)$ is of the $\operatorname{FIGARCH}\left(1, b_{i}, 1\right)$ type, augmented with a time-varying intercept; consistent with the findings of the structural break analysis, the latter is allowed to show a factor structure, with two common break processes in variance, i.e., the time-varying intercept component $w_{i, t}$ for the $i$ th generic conditional variance process is specified as

$$
w_{i, t}=\left[\begin{array}{ll}
\Lambda_{i, 1} & \Lambda_{i, 2}
\end{array}\right]\left[\begin{array}{l}
g_{1, t} \\
g_{2, t}
\end{array}\right],
$$

where the common break processes $g_{j, t}$ and factor loadings $\Lambda_{i, j}, j=1,2$, are also estimated by PCA implemented within the iterative procedure followed for the maximization of the log-likelihood function.

As shown in Table 8, the final estimates of the common stochastic and deterministic factors obtained for the conditional mean model are comparable with their starting estimate, both in terms of proportion of explained variance, total and for each series, as well as in terms of their interpretation as level, slope and curvature factors.

In fact, the first principal component accounts for the bulk of total variance for the OIS spreads (not normalized) break-free series (75\%; column 1) and the estimated break processes (95\%; column 4), and for no less than $70 \%$ of the variance for the 2 -month and longer maturities; the latter components are loaded with the same sign across the term structure, consistent with their level factor interpretation (trend -break process- and persistent deviation about trend -long memory component).

Moreover, the second principal component accounts for residual $13 \%$ and $4 \%$ of total variance for the (not normalized) break-free series and estimated break processes, respectively, yet explaining a sizable proportion of variance for each of the shortest maturities, i.e., about $40 \%$ for the 1-week, 2 -week and 1-month OIS spreads (columns 2 and 5); the latter components are loaded with opposite signs at the short- and medium- to long-end of the OIS spreads term structure, consistent with their slope factor interpretation.

In addition, the third principal component accounts for residual commonalities involving some of the maturities at the short-end of the term structure only; the latter is loaded with opposite signs at the short-/long- and mediumend of the OIS spreads term structure, consistent with its curvature factor interpretation. By adding to the estimated common long memory factors conditional mean, obtained from (2), the corresponding estimated common break process, the overall estimate of the level, slope and curvature factors is finally obtained; for instance, the level factor is computed by adding to the estimated conditional mean for the first common long memory factor the 
first estimated common break process; the slope and curvature factors are then obtained analogously.

Also, the first two principal components extracted from the common long memory factors conditional variance break processes $\left(w_{i, t}\right)$, consistent with the findings for the OIS spreads volatility proxies, account for $100 \%$ of their total variance (Table 8); while the former component explains $84 \%$ to $99 \%$ of the variance for the level, slope and curvature factor long-term (trend) conditional variances, the latter accounts for up to $16 \%$ of the variance for the level factor long-term conditional variance only. According to their estimated loadings, an interpretation in terms of level and slope factors for the OIS spreads variance term structure can then be provided to the estimated common break processes in variance.

Finally, from the estimation of the FIGARCH part of the model, consistent with the results of the long memory analysis, strong evidence of persistence in variance can be found for the three common long memory factors; a fractional differencing parameter $\left(b_{i}\right)$ in the range 0.30 through 0.40 is, in fact, estimated for their conditional variance processes (Table 8). The higher persistence in variance detected by means of the FIGARCH model than by using the BBLP estimator is not surprising, due to the likely noisiness of the volatility proxies employed, which may impart a downward bias to the BBLP estimator.

The estimated level, slope and curvature factors, and their estimated conditional standard deviation, contrasted with the corresponding trend/permanent components, are plotted in Figure 8; as is shown in the plots, the estimated conditional mean and standard deviation factors well describe the effects of the subprime crisis, pointing to a persistent increase in the level factor and in its volatility during the financial turmoil triggered by the BNP Paribas event (August 8 2007) and Lehman Brothers bankruptcy (September 15 2008), as well as in the volatility of the slope and curvature factors. Some permanent effects of the crisis (up to the end of the investigated sample, i.e., August 3 2012) can also be noted in the plots, as the trend component for all the term structure factors has not reverted to pre-crisis levels, while deviations about trend have become both less volatile and more persistent. Moreover, as shown by the (smoothed) conditional correlations (versus the 1-week OIS spread) plotted in Figure 9, the crisis also had the effect of decreasing the comovement between the short- and long-end of the OIS spreads term structure, ${ }^{21}$ as reported in Table 9 (Panel B), as a statistically significant contraction in the average conditional correlation coefficient for the post-crisis period, versus the crisis period, can indeed be found for the shortest OIS spread maturities,

\footnotetext{
${ }^{21} \mathrm{~A}$ full set of results is available upon request from the author.
} 
i.e., 1-week through 3-month, versus the longest ones, i.e., 7-month/9-month through 1-year.

\subsubsection{A fragility indicator}

Recent contributions to the literature on coincident (thermometer) and forwardlooking (barometer) financial fragility/risk indicators have focused on credit risk measures, rather than credit volumes gaps, which are more useful for real-time prediction. For instance, Schwaab et al. (2011) provide a systemic credit risk measure, contrasting the actual realization of a default probilitybased indicator for the financial sector with its predicted value according to macro-financial fundamentals. Bagliano and Morana (2011, 2012) propose a financial fragility indicator, based on the common component in the TED, agency and corporate spreads ${ }^{22}$, yielding a systemic credit risk measure as well, reflecting the state of the Government, Governmental Agencies, and private sector bond markets. Moreover, while an improved measure of the corporate spread is provided by Gilchrist and Zakrajsek (2012), Cassola and Morana (2012) propose a decomposition of the euro area OIS spreads term structure in level, slope and curvature factors, yielding insights on the three interrelated features of funding/hoarding liquidity risk, credit/counterparty risk, and investor confidence.

In Figure 10, the OIS spreads level factor $(L E V)$, the TED spread (TED), the $B A A-A A A$ spread $(C O R)$ and the mortgage spread $(M O R)^{23}$ are plotted over time; the shaded areas in the plots correspond to the most recent US recession, as dated by NBER's Business Cycle Dating Committee, i.e., December 2007 through June 2009, and the euro area crisis, decomposed into three subperiods, marked by the negative assessment by the EU-IMF of Greece's public finances in February 2010, the spreading of the crisis to Portugal, Spain and Ireland by November 2010, and its spillover to Italy by September 2011. The data source is FRED2.

As shown in the plots all the spread indicators appear to be informa-

\footnotetext{
${ }^{22}$ The TED spread, i.e., the spread between the 3 -month LIBOR rate (Euro dollar deposit rate) and the yield on 3-month Treasury bills, being the difference between an unsecured deposit rate and a risk-free rate, yields a measure of credit and liquidity risk; differently, the spread between $B A A$-rated and $A A A$-rated corporate bonds $(B A A-A A A)$ yields a measure of corporate default risk, as well as a measure of investors' risk-taking attitude, a contraction in the corporate spread signalling an increase in the demand for riskier bonds relative to safer ones; moreover, the agency spread is the spread between the 30-year Fannie Mae/Freddie Mac bonds yield and the 30-year Treasury bonds yield, measuring stress in the mortgage market.

${ }^{23}$ The mortgage spread is the spread between the conventional 30-year mortgage rate and 30-year Treasury bonds yield, measuring stress in the mortgage market.
} 
tive concerning the dating of the recession ensuing from the subprime crisis; in particular, from eyeball inspection, $T E D$ and $L E V$ show some leading indicator property, sharply increasing before its beginning; however, $L E V$, albeit strongly correlated with TED (the correlation coefficient for the two series is 0.65 ), does appear to contain also different information, pointing, for instance, to persisting stress in the interbank market over the period March through May 2009, not signalled by the TED; overall, $L E V, M O R$ and $T E D$ date quite closely the end of the US recession, while $C O R$ lags somewhat behind. Differently from the other measures, $L E V$ also shows some coincident indictor properties for the EA sovereign debt crisis, particularly concerning its beginning in February 2010 and its transmission to Italy in September 2011.

In Figure 10 (top plot), a composite fragility measure (FRAG), computed as the common component in the $L E V, T E D, C O R$ and $M O R$, i.e., their first principal component, is also plotted. ${ }^{24}$ The latter accounts for $80 \%$ of total variance, and for $44 \%(L E V)$ to $71 \%(C O R)$ of the variance for each individual series. By reflecting several dimensions of economic and financial fragility, i.e., interbank market stress-credit/liquidity risk and mortgage market and corporate sector conditions, the latter might also be useful as risk barometer.

The forward-looking properties of the proposed indicators are assessed by means of an out of sample forecasting exercise, with reference to their ability of predicting industrial production, inflation and unemployment rate dynamics. Different horizons are considered, i.e., 1-month, 3-month, 6-month and 12-month, while the forecasting sample is from August 2007 through July 2012. In order to make macroeconomic and risk indicators frequencies consistent, the OIS spreads term structure level, slope and curvature factors are firstly computed recursively using daily data; then, a monthly figure is computed by averaging the daily figures over the calendar month. Averaging over calendar month is also employed for the TED, COR and MOR indicators.

Forecasts for the macroeconomic variables of interest are computed by means of VAR models, using different specifications, i.e., the $F$ model, including the estimated level $(L E V)$, slope and curvature factor conditional means; the $F 1$ model, including $L E V$ only; the $F 2$ model, including the estimated slope factor conditional mean only; the $F 3$ model including the estimated curvature factor conditional mean only; the $C$ model including the composite indicator $(F R A G)$ constructed from the common component in $L E V, T E D, C O R$ and $M O R$; the $C F$ model, including FRAG and the

\footnotetext{
${ }^{24}$ The estimated weights are 1.194, 1.214, 0.622 and 1.078 for the $L E V, C O R, T E D$ and MOR series, respectively.
} 
estimated slope and curvature factors conditional means. The above models are contrasted with other VAR specifications, considering alternative risk measures to OIS spreads term structure factors; in particular, the $A 1$ model, including the corporate spread $(C O R)$; the $A 2$ model including the TED spread; the $A 3$ model including the mortgage spread (MOR); the $B$ model, including the Federal funds rate and the term spread (computed using 10year and 3-month Treasury constant maturity rate bonds and bills); the $B 1$ model, including the Federal funds rate only; the $B 2$ model, including the term spread only; finally, the "no change" forecasting model $(N A I V E)$ and an autoregressive model $(A R)$ for the macroeconomic variables of interest are considered as well.

The assessment of the forecasting properties of the various models is performed by means of the root mean square forecast error (RMSFE) and the Theil's inequality $(I C)$ statistics. In order to make conclusions robust to lag selection, forecasts are generated from specifications containing up to 5 lags; then, the best outcome for each forecasting model, across dynamic specifications, is reported in Table 10 for any horizon. Note that, by comparing the performance of the various VAR models against the AR model, the excess information contained in the proposed indicators, relatively to the past history of the own macroeconomic variables, can be assessed.

As shown in Table 10 (Panel A), concerning the prediction of unemployment rate dynamics, the $C$ model performs best at the 1- and 3-month horizons, while the $C F$ model yields the best outcome at longer horizons; yet, while the $I C$ statistic selects $C F$ as best model also at the 1-year horizon, the $A 2$ model is selected according to the RMSFE statistics. The $C$ and $C F$ models finally yield the most synchronous forecasts, in terms of correlation coefficient, with actual unemployment rate dynamics. Finally, the excess information content of the proposed risk indicators can be easily gauged by comparing $C, C F$ and $A R$ model figures: a $5 \%$ to $50 \%$ reduction in the $I C$ and $R M S F E$ statistics can be noted across forecasting horizons, therefore pointing to the usefulness of the proposed indicators.

Moreover, also concerning industrial production dynamics (Table 10, Panel B), VAR models containing OIS spreads term structure information perform best: according to the RMSFE statistic, F2 at the 1-, 6- and 12-month horizons; $F$ at the 3 -month horizon; according to the $I C$ statistic, $F$ at the 3 - and 6-month horizon; $F 2$ at the 12-month horizon; $C F$ at the 1-month horizon. Also sizable is the excess information provided by the proposed credit risk indicators: $5 \%$ to $30 \%$ reductions in the $I C$ and $R M S F E$ statistics can be noted across horizons; moreover, also very sizable is the correlation between actual and forecasted values using the $F, F 2$ and $C F$ models.

Finally, interesting results are also obtained for inflation rate forecasting 
(Table 10, Panel C) at short horizons, as $F 3$ and $C F$ perform best at the 1-month horizon according to the $R M S F E$ and $I C$ statistics, respectively; a $20 \%$ reduction in the $R M S F E$ and $I C$ statistics, relatively to $A R$ model figures, and fairly correlated forecasted and actual values, can also be noted.

Overall, the above findings look promising concerning the real-time use of the proposed indicators for macroeconomic forecasting and as risk barometer.

\subsection{Impulse responses and forecast error variance de- composition}

Due to instability in variance, impulse response analysis and forecast error variance decomposition have been made dependent on the estimated volatility regimes, i.e., structural common factor shocks have been computed using the estimated variance-covariance matrix $\hat{\Sigma}_{\eta, s}$, where $s=$ pre-crisis, crisis, post-crisis. As the orthogonality of the common factors is imposed over the full sample, and therefore does not necessarily hold over each subsample, the identification of the structural common factor shocks, and therefore the estimation of the $H$ matrix ( $H_{s}$, being regime dependent) in the structural VMA representation of the FI-HF-VAR model in (25) requires 3 additional restrictions $(r(r-1) / 2 ; r=3)$, which are imposed through a recursive specification for the structural form of the system of equations in (2), assuming the level factor ordered first and the curvature factor last; then, the $H_{s}$ matrix is estimated by means of the Choleski decomposition of the contemporaneous variance-covariance matrix of the reduced form common factor innovations, yielding $\hat{H}_{s}=\operatorname{chol}\left(\hat{\Sigma}_{\hat{\eta}, s}^{-1}\right)$.

Due to the possible dependence of the results on the selected ordering, impulse responses are also carried out by assuming a diagonal structure for the $\hat{\Sigma}_{\eta, s}$ matrix and, therefore, for the matrix $H_{s}$, as it would be implied by the orthogonality of the common factors over each subsample; the results of the impulse response analysis obtained from the latter model are fully coherent, in terms of sign, profile and magnitude, with those obtained by means of the recursive structure, which is evidence of robustness of the findings to identifying restrictions. ${ }^{25}$.

Moreover, the identification of the idiosyncratic shocks requires additional 91 restrictions $(n(n-1) / 2 ; n=14)$, which are similarly imposed by selecting

\footnotetext{
${ }^{25}$ In terms of magnitude of the median contemporaneous impact, absolute deviations no larger than 0.4b.p., 0.09 b.p. on average, are found for the OIS spreads responses to the slope factor shock; figures for the curvature factor shock are 1b.p. for the median impact and 0.2b.p. on average. By construction, no differences for the responses to the slope factor shock are found for the two identification strategies. Detailed results are not reported for reasons of space; they are however available upon request from the author.
} 
a recursive structure for the system of equations in (1). The latter assumes the 1-week rate spread ordered first and the 1-year spread ordered last, and therefore contemporaneous forward transmission of shocks along the OIS spreads term structure, yet only delayed (one-day at least) feedback from longer to shorter maturities. Hence, the $\Theta$ matrix in the structural VMA representation of the FI-HF-VAR model in (25) is estimated by means of the Choleski decomposition of the contemporaneous variance-covariance matrix of the idiosyncratic innovations, i.e., $\hat{\Theta}^{-1}=\operatorname{chol}\left(\hat{\Sigma}_{\hat{v}}\right)$.

\subsubsection{Impulse response analysis}

The results of the impulse response analysis are reported in Figure 10-12, where median impulse responses, with $90 \%$ significance bands, are plotted for the three regimes investigated, over a twenty-five-day horizon; for reasons of space, only selected maturities, i.e., 1-week, 1-month, 3-month, 6-month, 9-month and 1-year, are considered. ${ }^{26}$

As shown in the figures, independently of the regime considered, the interpretation of the structural common persistent shocks in terms of level, slope and curvature factor shocks is supported by the results of the impulse response analysis; in fact, a 1-standard deviation level shock drives upwards the whole OIS spreads term structure (Figure 10), while responses of opposite sign can be noted at the short- and medium-/long-end of the OIS spreads term structure, following a 1-standard deviation slope shock; moreover, responses of opposite sign can be noted at the short-/long-end of the OIS spreads term structure, relatively to intermediate maturities, following a 1-standard deviation curvature shock.

Consistent with the finding of long memory in the common stochastic factors, the effects of the level, slope and curvature factor shocks tend to fade away slowly, showing a hyperbolic rate of decay, being still statistically significant also after twenty days. By comparing impulse responses to each shock across regimes, it can be noted that the subprime crisis has lead to an increase in the persistence of all the common shocks, enduring also after its end.

Moreover, the crisis also had the effect of magnifying the contemporaneous impact of all the common shocks. For instance, for the level shock, a three to five fold larger effect can be noted for the crisis regime (7b.p. to 11b.p.) relatively to the pre-crisis regime (1.7b.p. to 2.6b.p), while a two to three fold larger effect can be noted for the post-crisis period (5b.p, to 7.5b.p.); results for the other common persistent shocks are similar, i.e., -

\footnotetext{
${ }^{26} \mathrm{~A}$ full set of results is available from the author upon request.
} 
0.4b.p. to 0.75b.p., -1b.p. to 3b.p., and -1b.p. to 2b.p., for the pre-crisis, crisis, and post-crisis periods, respectively, for the slope factor shock; figures for the curvature factor shock are -0.2b.p. to 1.5b.p., -0.8b.p. to 5b.p. and -0.5b.p. to 3b.p., for the three regimes, respectively.

\subsubsection{Forecast error variance decomposition}

The results of the median forecast error variance decomposition (FEVD) are reported in Table 11. For reasons of space only a selection of the results is reported, i.e., FEVD at the 1-day and 20-day horizons, for the three regimes considered. ${ }^{27}$

As shown in Table 11, independently of the regime considered, some conclusions can be drawn concerning the relevance of money market shocks.

Firstly, the slope factor shock is most important at the short-end of the term structure, independently of the horizon considered (1-day or 20-day); the latter also accounts for some fluctuations at the long-end of the term structure.

Secondly, the level factor shock is the key driver of OIS spreads fluctuations from the 2-month maturity onwards, playing a stronger role at longer (20-day) than shorter (1-day) horizons.

Thirdly, the curvature factor shock accounts for fluctuations common to intermediate maturities, i.e., 2-month to 4-month, as well as to the short(1-week) and long- (1-year) end of the term structure.

Fourthly, idiosyncratic fluctuations are more important at the short-end of the term structure than for longer maturities.

Additional interesting findings, related to the consequences of the subprime financial crisis, can also be noted.

Firstly, while the impact (1-day) contribution of the slope factor shock to short-end term structure fluctuations (1-week through 1-month maturities) is fairly unchanged, i.e., $30 \%$, a sizable increase can be noted at longer horizons (20-day), i.e., from $40 \%$ (pre-crisis) to $54 \%$ (crisis) and then $60 \%$ (post-crisis); similarly, in relative terms, for the 2-week and 1-month maturities.

Secondly, independent of the maturity and horizon, the contribution of the level factor shock to OIS spreads fluctuations has increased across regimes, more sizably during the crisis than post-crisis periods, and for short/long maturities than for intermediate maturities (in terms of relative changes); for instance, the contribution of the level factor shock to 1-week OIS spread fluctuations (20-day horizon) has increased from $20 \%$ (pre-crisis) to $30 \%$ (crisis) and then $32 \%$ (post-crisis); figures for the 1-year maturity are

\footnotetext{
${ }^{27} \mathrm{~A}$ full set of results are available from the author upon request.
} 
$63 \%, 86 \%$ and $88 \%$, while for the 4-month maturity are $82 \%, 95 \%$ and $97 \%$, respectively.

Thirdly, the increasing contribution of the level factor shock to OIS spreads fluctuations across regimes has been matched by a progressive contraction in the contribution of the other shocks, i.e., slope and curvature factors and idiosyncratic; in particular, the decreased contribution of the slope and curvature factor shocks, accounting for some common fluctuations at the short- and long-end of term structure, may explain their declining comovement detected by the conditional correlation analysis (Table 10). Overall, the crisis appears to have magnified the role of persistent and common shocks over idiosyncratic shocks, and, among the former, the contribution of level over slope and curvature factor shocks.

\section{Conclusions}

In the paper the fractionally integrated heteroskedastic factor vector autoregressive (FI-HF-VAR) model is introduced. The proposed approach shows minimal pretesting requirements, performing well independently of integration properties of the data and sources of persistence, i.e., deterministic or stochastic, accounting for common features of different kinds, i.e., common integrated (of the fractional or integer type) or non integrated stochastic factors, also heteroskedastic, and common deterministic break processes. Consistent and asymptotically normal estimation, by means of an iterative multistep algorithm, similar to Stock and Watson (2005), can be conjectured. The iterative procedure may also be augmented by an additional step based on the Granger and Jeon (2004) thick modelling strategy, providing median estimates of the parameters of interest and robust standard errors. Moreover, the approach is easy to implement and effective also in the case of very large systems of dynamic equations. An extensive Monte Carlo analysis strongly supports the proposed methodology.

The dynamic properties of US OIS spreads, over the subprime and euro area sovereign debt crises, are investigated by means of the proposed approach. Among the main empirical results, we find that three common components, bearing the interpretation of level, slope and curvature factors, can be extracted from the OIS spreads term structure; the latter are characterized by deterministic trend component and strongly persistent and heteroskedastic fluctuations about trend; two common break processes, describing the long-term evolution of OIS spreads conditional variances, bearing the interpretation of level and slope factors for the volatility term structure, are also found. 
Moreover, the subprime crisis has lead to a wide increase in both the mean and variance of OIS spreads trend levels and to a sizable increase in the persistence of money market shocks; while at the short-end of the term structure mean and variance trend components have progressively converged back to pre-crisis levels, at the medium- long-end fluctuations about much higher mean values, than prevailing before the crisis, have persisted over the post-crisis period as well; moreover, persistence of money market shocks has not reverted back to pre-crisis intensity, actually even further increasing over the post-crisis period. A sizable increase in OIS spreads mean trend level at the medium- to long-end of the term structure can also be associated with the beginning of the euro area sovereign debt crisis and its spillover to the Italian economy. Should wide OIS spreads become a long-lasting feature of the US money market, surely important challenges for theoretical models of the yield curve and for the pricing of interest rate and credit derivatives would then raise.

By finally comparing the forward-looking properties of the OIS spreads term structure factors with alternative measures of credit/liquidity risk and financial fragility, we find the former conveying additional information, relatively to commonly used measures like the $T E D$ or the $B A A-A A A$ corporate spreads, which might be exploited, also within a composite indicator, for the construction of a risk barometer and real-time macroeconomic forecasting.

To our knowledge no such a comprehensive study on the consequences of the subprime and euro area sovereign debt crisis on the US money market has previously been contributed to the literature; the proposed modeling framework does appear to be needed for an accurate modeling of OIS spreads empirical properties and the understanding of the recent financial turmoil. Other empirical implementations, involving for instance the modeling of realized moments of financial returns and macroeconomic variables, can be envisaged, and suggestions for further applications are provided as well.

\section{References}

[1] Acharya, V.V. and M.P. Richardson, 2009, Causes of the financial crisis, Critical Review, 21, 195-210.

[2] Afonso, G., A. Kovner and A. Scholar, 2011, Stressed, not frozen: The Federal funds market during the financial crisis, Federal Reserve of New York Staff Report, no. 437.

[3] Alexander, C.O., 2002, Principal component models for generating large covariance matrices, Economic Notes, 31, 337-359. 
[4] Allen, F., E. Carletti and D. Gale, 2009, Interbank market liquidity and central bank intervention, Journal of Monetary Economics, 56, 639-652.

[5] Amado, C. and T. Terasvirta, 2008, Modelling conditional and unconditional heteroskedasticity with smoothly time-varying structure, CREATES Research Paper, no. 8.

[6] Armantier, O., J.J. McAndrews and S. Krieger, 2008, The Federal Reserve's term auction facility, Federal Reserve Bank of New York Current Issues in Economics and Finance, 14, Number 5.

[7] Baillie, R.T., 1996, Long memory processes and fractional integration in econometrics, Journal of Econometrics, 73, 5-59.

[8] Baillie, R.T., T. Bollerslev and H.-O. Mikkelsen, 1996, Fractionally integrated generalized autoregressive conditional heteroskedasticity, Journal of Econometrics, 74, 3-30.

[9] Baillie, R.T. and C. Morana, 2009, Modeling long memory and structural breaks in conditional variances: An adaptive FIGARCH approach, Journal of Economic Dynamics and Control, 33, 1577-1592.

[10] Baillie R.T. and C. Morana, 2012, Adaptive ARFIMA Models with Applications to Inflation, Economic Modelling, 29, 2451-2459.

[11] Bagliano, F.C. and C. Morana, 2012, Determinants of US financial fragility conditions, 2012, Research in International Business and Finance, forthcoming.

[12] Bai, J. and P. Perron, 1998, Testing for and estimation of multiple structural changes, Econometrica, 66, 47-79.

[13] Bai, J. and S. Ng, 2002, Determining the number of factors in approximate factor models, Econometrica, 70, 191-221.

[14] Bai, J., 2003, Inferential theory for factor models of large dimensions, Econometrica, 71, 135-171.

[15] Bai, J., 2004, Estimating cross-section common stochastic trends in nonstationary panel data, Journal of Econometrics, 122, 137-38.

[16] Bai, J. and S. Ng, 2004, A panic attack on unit roots and cointegration, Econometrica, 72, 1127-1177. 
[17] Bai, J. and S. Ng, 2006, Confidence intervals for diffusion index forecasts and inference with factor-augmented regressions, Econometrica, $74,1133-1150$.

[18] Bai, J. and S. Ng, 2007, Determining the number of primitive shocks in factor models, Journal of Business and Economic Statistics, 25, 52-60.

[19] Bai, J. and S. Ng, 2008, Forecasting economic time series using targeted predictors, Journal of Econometrics, 146, 304-317.

[20] Becker, R., W. Enders and J. Lee, 2006, A stationarity test in the presence of an unknown number of smooth breaks, Journal of Time Series Analysis, 27, 381-409.

[21] Bianco, K, 2012, A retrospective of the troubled asset relief program, Wolters Kluwer Law \& Business, mimeo.

[22] Bierens, H.J., 2000, Non parametric nonlinear cotrending analysis, with an application to interest and inflation in the United States, Journal of Businness and Economic Statistics, 18, 323-37.

[23] Breitenfellner, B. and N. Wagner, 2010, Government intervention in response to the subprime financial crisis: The good into the pot, the bad into the crop, Passau University, mimeo.

[24] Brunnermaier, M., 2009, Deciphering the liquidity crisis, Journal of Economic Perspectives, 23, 77-100.

[25] Brunetti, C. and C.L. Gilbert, 2000, Bivariate FIGARCH and fractional cointegration, Journal of Empirical Finance, 7, 509-530.

Bollerslev, T., 1986, Generalized autoregressive conditional heteroskedasticity, Journal of Econometrics, 31, 307-27.

[26] Bollerslev, T., 1990, Modelling the coherence in short-run nominal exchange rates: a multivariate generalized ARCH approach. Review of Economics Studies 72, 489-505.

[27] Caballero, R.J. and A. Krishnamurthy, 2008, Collective risk management in aflight to quality episode, Journal of Finance, 63, 2195-2230.

[28] Cassola, N. and C. Morana, 2012, Euro money market spreads during the 2007-? financial crisis, Journal of Empirical Finance, 19, 548-557.

[29] Chan, N.H and W. Palma, 1998, State space modelling of long memory processes, Annals of Statistics, 26, 719-740. 
[30] Christensen, J.H.E., 2009, Have the Fed liquidity facilities had an effect on Libor?, FRBSF Economic Letter, 2009, issue Aug 10.

[31] Christensen, J.H.E., J.E. Lopez and G.D. Rudebusch, 2009, Do central bank liquidity facilities, Federal Reserve Bank of San Francisco Working Paper.

[32] Connor, G. and R. Korajzcyk, 1993, A test for the number of factors in an approximate factor model, Journal of Finance, 48, 1263-1291.

[33] Conrad, C. and B.R. Haag, 2006, Inequality constraints in the fractionally integrated GARCH model, Journal of Financial Econometrics 4, 413-449.

[34] Chung, C.-F., 1999. Estimating the fractionally integrated GARCH model. Working Paper, National Taiwan University.

[35] Cragg, J. and Donald, S., 1997, Inferring the rank of a matrix, Journal of Econometrics, 76, 223-50.

[36] D'Amico, S., W. English, J.D. Lopez-Salido and E. Nelson, 2012, The Federal Reserve's large-scale asset purchase programs: Rational and effects, CEPR Discussion Papers, no. 9145.

[37] Dees, S., M.H. Pesaran, L.V. Smith, and R.P. Smith, 2010, Supply, demand and monetary policy shocks in a multi-country New Keynesian model, ECB Working Paper Series, no. 1239.

[38] Demetrescu, M., Kuzin, V. and U. Hassler, 2006, Long memory testing in time domain, Goethe University Frankfurt, mimeo.

[39] Dempster, A.P., Laird, N.H. and D.B. Rubin, 1977, Maximum likelihood from incomplete data via the EM algorithm, Journal of the Royal Statistical Society, Series B, 39, 1-38.

[40] De Santis, R.A., 2012, The euro area sovereign debt crisis. Safe haven, credit rating agencies and the spread of the fever from Greece, Ireland and Portugal, ECB Working Paper Series, no. 1419.

[41] Diebold, F.X. and A. Inoue, 2001, Long memory and regime switching, Journal of Econometrics, 105, 131-59.

[42] Dolado, J.J., J. Gonzalo and L. Mayoral, 2005, A simple test of long memory vs. structural breaks in the time domain, Universidad Carlos III de Madrid, mimeo. 
[43] Doz, C., D. Giannone, and L. Reichlin, 2011, A Two-step estimator for large approximate dynamic factor models based on Kalman filtering, Journal of Econometrics, 164, 188-205.

[44] Engle, R.F and T. Bollerslev, 1986, Modelling the persistence of conditional variances, Econometric Reviews, 5, 1-50.

[45] Engle R.F. , 2002, Dynamic Conditional Correlation - A simple class of multivariate GARCH models, Journal of Business and Economic Statistics, 20, 339-350.

[46] Engle, R.F. and J.C. Rangel, 2008, The spline-GARCH model for low frequency volatility and its global macroeconomic causes. Review of Financial Studies, 21, 1187-1222.

[47] Engle R.F. and B.T. Kelly, 2008, Dynamic Equicorrelation, NYU Working Paper, no. FIN-08-038.

[48] Enders, W. and J. Lee, 2004, Testing for a unit-root with a non linear Fourier function, University of Alabama, mimeo.

[49] Frank, N. and H. Hesse, 2009, The effectiveness of central bank interventions during the first phase of the subprime crisis, IMF Working Paper, no. 206.

[50] Forni M., M. Hallin, M. Lippi, and L. Reichlin, 2000, The generalized dynamic factor model: identification and estimation, Review of Economics and Statistics, 82, 540-554.

[51] Gallant, A R., 1984, The Fourier flexible form, American Journal of Agricultural Economics, 66, 204-08.

[52] Geweke, J., 1977, The dynamic factor analysis of economic time series, in D. J. Aigner and A. S. Goldberger (eds.), Latent Variables in SocioEconomic Models, Amsterdam: North-Holland.

[53] Granger, C.W. and Y. Jeon, 2004, Thick modelling, Economic Modelling, 21, 323-43.

[54] Granger, C.W.J and N. Hyung, 2004, Occasional structural breaks and long memory with an application to the S\&P500 absolute returns, Journal of Empirical Finance, 11, 399-421.

[55] Gilchrist, S. and E. Zakrajšek, 2012, Credit spreads and business cycle fluctuations, American Economic Review, 102, 1692-1720. 
[56] Gill, L. and A. Lewbell, 1992, Testing the rank and definitess of estimated matrices with applications to factor, state space and ARMA models, Journal of the American Statistical Association, 87, 766-776.

[57] Gonzalez, A., Hubrich, K., and T. Terasvirta, 2009. Forecasting inflation with gradual regime shifts and exogenous information, CREATES Research Paper 2009-3.

[58] Hassler, U. and B. Meller, 2009, Detecting a change in inflation persistence in the presence of long memory: A new test, Goethe University Frankfurt, mimeo.

[59] Hamilton, J. D., 1989, A new approach to the economic analysis of nonstationary time series and the business cycle, Econometrica, 57, 357384.

[60] Hamilton, J.D., Susmel, R., 1994, Autoregressive conditional heteroskedasticity and changes in regime, Journal of Econometrics, 64, 30733.

[61] Jackson, D.A., 1993, Stopping rules in principal components analysis: A comparison of heuristical and statistical approaches, Ecology, 74, 22042214 .

[62] Kapetanios, G, 2010, A testing procedure for determining the number of factors in approximate factor models wth large datasets, Journal of Business and Economic Statistics, 28, 397-409.

[63] Kilian, L., 2011, Structural vector autoregressions, CEPR Discussion Paper, no. 8515.

[64] Krishnamurthy, A. and A. Vissing-Jorgessen, 2011, The effects of quantitative easing on interest rates: Channels and implications for policy, NBER Working Paper Series, no.17555.

[65] Martin, L.V. and N.P. Wilkins, 1999, Indirect estimation of ARFIMA and VARFIMA models, Journal of Econometrics, 93, 149-75.

[66] McCloskey, A. and P. Perron, 2011, Memory parameter estimation in the presence of level shifts and deterministic trends, mimeo, Boston University.

[67] Morana, C., 2007, Multivariate modelling of long memory processes with common components, Computational Statistics and Data Analysis, 52, 919-934. 
[68] Morana, C., 2009, On the macroeconomic causes of exchange rate volatility, International Journal of Forecasting, 25, 328-350.

[69] Morana, C., 2012, Oil price dynamics, macro-finance interactions and the role of financial speculation, Journal of Banking and Finance, forthcoming.

[70] Moulines, E. and P. Soulier, 1999, Broadband log-periodogram regression of time series with long range dependence, The Annals of Statistics, 27, 1415-39.

[71] Ohanissian, A., J. R. Russell and R. S. Tsay, 2008, True of spurious long memory? A new test, Journal of Business and Economic Statistics, 26, 161-175.

[72] Paul, S.R., 1989, Test for the equality of several correlation coefficients, The Canadian Journal of Statistics, 17, 217-227.

[73] Perron, P., 1989, The great crash, the oil price shock and the unit root hypothesis, Econometrica, 57, 1361-1401.

[74] Perron, P. and Z. Qu, 2010, Long memory and level shifts in the volatility of stock market return indices, Journal of Business and Economic Statistics, 28, 275-290.

[75] Pesaran, M.H. and R.S. Smith, 2011, Beyond the DSGE straitjacket, Discussion Paper, no.5661, Cambridge University.

[76] Phillips, P. C. B. and K. Shimotsu, 2004, Local Whittle estimation in nonstationary and unit root cases, Annals of Statistics, 32, 656-692.

[77] Reis, R., 2009, Interpreting unconventional US monetary policy, 2009, Brooking Papers on Economic Activity, Fall, 119-182.

[78] Robin, J.M. and R.J. Smith, 2000, Test of rank, Econometric Theory, $16,151-75$.

[79] Robinson, P.M., 2006, Conditional-sum-of-squares estimation of models for stationary time series with long momory, IMS Lecture NotesMonograph Series, Time Series and Related Topics, 52, 130-137.

[80] Schwaab, B., S.J. Koopman, and A. Lucas, 2011, Systemic risk diagnostics. Coincident indicators and early warning signals, ECB Working Paper Series, no.1327. 
[81] Schwert, G.,W., 1989, Test for unit roots: A Monte Carlo investigation, Journal of Business and Economic Statistics, 7, 147-160.

[82] Shimotsu, K., 2006, Simple (but effective) tests of long memory versus structural breaks, Working Paper, no. 1101, Department of Economics, Queen's University.

[83] Sowell, F., 1992, Maximum likelihood estimation of fractionally integrated time series models, Journal of Econometrics, 53, 165-88.

[84] Stock J.H. and M.W. Watson, 1998, Diffusion indexes, NBER Working Paper, no. 6702.

[85] Stock J.H. and M.W. Watson, 2005, Implications of dynamic factor models for VAR analysis, NBER Working Paper, no. 11467.

[86] Stock J.H. and M.W. Watson, 2011, Dynamic factor models, in M.P. Clements and D.F. Hendry (eds.), Oxford Handbook of Economic Forecasting, Oxford University Press, 35-60.

[87] Taylor, J.B. and J.C. Williams, 2009, A black swan in the money market, American Economic Journal: Macroeconomics, 1, 58-83.

[88] Veronesi, P. and L. Zingales, 2009, Paulson's gift, NBER Working Paper No. 15458.

[89] Wu, T, 2011, The U.S. money market and the term auction facility in the financial crisis of 2007-2009, The Review of Economics and Statistics, 93, 617-631. 
Table 1: OIS spreads, Bai-Perron structural breaks tests.

\begin{tabular}{|c|c|c|c|c|c|c|c|c|}
\hline \multicolumn{9}{|c|}{ Panel A: OIS spreads levels } \\
\hline & & & $\operatorname{PrC}$ & $C 1$ & $C 2$ & PoC1 & $\mathrm{PoC2}$ & $\mathrm{PoC3}$ \\
\hline & UDmax & Break Points & Mean & mean & mean & mean & mean & mean \\
\hline$x^{1 w}$ & 39.9 & $8 / 9 / 07 ; 9 / 16 / 08 ; 12 / 9 / 08$ & 0.074 & 0.403 & 1.437 & 0.129 & 0.082 & 0.078 \\
\hline$x^{2 w}$ & 47.8 & $8 / 9 / 07 ; 9 / 16 / 08 ; 12 / 10 / 08$ & 0.079 & 0.446 & 1.565 & 0.172 & 0.088 & 0.102 \\
\hline$x^{1 m}$ & 47.9 & $8 / 9 / 07 ; 9 / 19 / 08 ; 12 / 12 / 08$ & 0.087 & 0.493 & 1.839 & 0.217 & 0.095 & 0.134 \\
\hline$x^{2 m}$ & 69.6 & 8/9/07; 9/19/08; 12/17/08; 8/24/09; 9/12/11 & 0.099 & 0.611 & 2.097 & 0.558 & 0.121 & 0.237 \\
\hline$x^{3 m}$ & 84.1 & $8 / 9 / 07 ; 9 / 19 / 08 ; 12 / 18 / 08 ; 8 / 24 / 09 ; 9 / 12 / 11$ & 0.108 & 0.692 & 2.210 & 0.770 & 0.151 & 0.354 \\
\hline$x^{4 m}$ & 105.3 & $8 / 10 / 07 ; 9 / 19 / 08 ; 12 / 17 / 08 ; 9 / 7 / 09 ; 9 / 9 / 11$ & 0.109 & 0.727 & 2.240 & 0.963 & 0.201 & 0.439 \\
\hline$x^{5 m}$ & 107.1 & $8 / 10 / 07 ; 9 / 19 / 08 ; 12 / 17 / 08 ; 9 / 10 / 09 ; 9 / 7 / 11$ & 0.112 & 0.759 & 2.270 & 1.108 & 0.261 & 0.511 \\
\hline$x^{6 m}$ & 128.6 & $8 / 14 / 07 ; 9 / 19 / 08 ; 12 / 17 / 08 ; 9 / 9 / 09 ; 9 / 12 / 11$ & 0.117 & 0.784 & 2.299 & 1.222 & 0.321 & 0.588 \\
\hline$x^{7 m}$ & 141.9 & $8 / 14 / 07 ; 9 / 19 / 08 ; 12 / 17 / 08 ; 9 / 10 / 09 ; 9 / 6 / 11$ & 0.118 & 0.772 & 2.284 & 1.271 & 0.379 & 0.642 \\
\hline$x^{8 m}$ & 165.6 & $8 / 10 / 07 ; 9 / 19 / 08 ; 12 / 17 / 08 ; 9 / 10 / 09 ; 9 / 9 / 11$ & 0.121 & 0.756 & 2.263 & 1.314 & 0.435 & 0.691 \\
\hline$x^{9 m}$ & 193.7 & $8 / 10 / 07 ; 9 / 19 / 08 ; 12 / 17 / 08 ; 9 / 11 / 09 ; 9 / 6 / 11$ & 0.122 & 0.740 & 2.241 & 1.351 & 0.489 & 0.740 \\
\hline$x^{10 m}$ & 217.7 & $8 / 9 / 07 ; 9 / 19 / 08 ; 12 / 17 / 08 ; 9 / 11 / 09 ; 9 / 6 / 11$ & 0.124 & 0.729 & 2.218 & 1.380 & 0.538 & 0.790 \\
\hline$x^{11 m}$ & 232.8 & 8/9/07; 9/19/08; 12/17/08; 9/11/09; 9/8/11 & 0.125 & 0.714 & 2.192 & 1.407 & 0.586 & 0.842 \\
\hline$x^{12 m}$ & 261.6 & $8 / 9 / 07 ; 9 / 19 / 08 ; 12 / 17 / 08 ; 9 / 11 / 09 ; 9 / 6 / 11$ & 0.125 & 0.701 & 2.164 & 1.432 & 0.635 & 0.898 \\
\hline
\end{tabular}

\begin{tabular}{|c|c|c|c|c|c|}
\hline \multicolumn{7}{|c|}{ Panel B: OIS spreads volatilities } \\
\hline & & & PrC & C & PoC \\
\hline & UDmax & Break Points & mean & mean & mean \\
\hline$\left|\Delta x^{1 w}\right|$ & 55.7 & $8 / 2 / 07 ; 1 / 2 / 09$ & 0.010 & 0.074 & 0.007 \\
\hline$\left|\Delta x^{2 w}\right|$ & 59.8 & $8 / 2 / 07 ; 12 / 23 / 08$ & 0.007 & 0.068 & 0.005 \\
\hline$\left|\Delta x^{1 m}\right|$ & 82.9 & $8 / 2 / 07 ; 12 / 22 / 08$ & 0.007 & 0.052 & 0.005 \\
\hline$\left|\Delta x^{2 m}\right|$ & 71.9 & $8 / 2 / 07 ; 2 / 2 / 09$ & 0.007 & 0.047 & 0.005 \\
\hline$\left|\Delta x^{3 m}\right|$ & 64.8 & $8 / 2 / 07 ; 2 / 2 / 09$ & 0.009 & 0.047 & 0.006 \\
\hline$\left|\Delta x^{4 m}\right|$ & 74.2 & $8 / 2 / 07 ; 1 / 3 / 09$ & 0.012 & 0.050 & 0.007 \\
\hline$\left|\Delta x^{5 m}\right|$ & 82.1 & $7 / 27 / 07 ; 2 / 11 / 09$ & 0.015 & 0.052 & 0.007 \\
\hline$\left|\Delta x^{6 m}\right|$ & 97.0 & $7 / 25 / 07 ; 2 / 11 / 09$ & 0.018 & 0.058 & 0.008 \\
\hline$\left|\Delta x^{7 m}\right|$ & 106.7 & $7 / 25 / 07 ; 2 / 3 / 09$ & 0.021 & 0.060 & 0.009 \\
\hline$\left|\Delta x^{8 m}\right|$ & 118.0 & $7 / 25 / 07 ; 2 / 11 / 09$ & 0.024 & 0.064 & 0.010 \\
\hline$\left|\Delta x^{9 m}\right|$ & 124.2 & $7 / 25 / 07 ; 2 / 9 / 09$ & 0.028 & 0.067 & 0.011 \\
\hline$\left|\Delta x^{10 m}\right|$ & 128.5 & $7 / 25 / 07 ; 1 / 28 / 09$ & 0.031 & 0.070 & 0.012 \\
\hline$\left|\Delta x^{11 m}\right|$ & 127.3 & $7 / 25 / 07 ; 2 / 2 / 09$ & 0.034 & 0.073 & 0.013 \\
\hline$\left|\Delta x^{12 m}\right|$ & 128.8 & $7 / 25 / 07 ; 2 / 2 / 09$ & 0.037 & 0.077 & 0.014 \\
\hline
\end{tabular}

The Table reports the results of the Bai-Perron (1989) UDmax test, carried out using monthly OIS spreads levels and volatilities over the period May 2002 through July 2007, and the estimated break points using daily data. The daily volatility proxy is computed from the absolute first differences of the OIS spreads levels $(|\Delta x|)$. Mean values for the series over the various regimes, computed using daily data, are also reported: Pre-crisis: 5/6/02 - 8/8/07 (PrC), Crisis: pre-Lehman 8/9/07 - 9/15/08 (C1), Crisis: post-Lehman 9/16/08 -12/8/08 (C2), Post-crisis I: 12/9/08 - 8/21/09 (PoC1), Post-crisis II: 8/24/09- 9/5/11 (PoC2), Post-crisis III: EA crisis 9/6/11-8/3/12 (PoC3). The results are reported for the various OIS spreads maturities available, i.e., from 1-week $\left(x^{1 w}\right)$ to one-year $\left(x^{12 m}\right)$. 
Table 2: Full sample estimates of the fractional differencing parameter and long memory tests: OIS spreads levels and volatilities

\begin{tabular}{|c|c|c|c|c|c|c|c|c|c|c|c|}
\hline \multicolumn{6}{|c|}{ Panel A: OIS spreads levels } & \multicolumn{6}{|c|}{ Panel B: OIS spreads volatilities } \\
\hline & level $(x)$ & break-free (l) & SKPSS & $D G M$ & DKH & & level $(|\Delta x|)$ & break-free (v) & SKPSS & DGM & DKH \\
\hline$x^{1 w}$ & $0.885(0.039)$ & $0.519(0.039)$ & 0.021 & -2.773 & -2.233 & $\left|\Delta x^{1 w}\right|$ & $0.308(0.039)$ & $0.175(0.039)$ & 0.571 & 0.174 & 0.181 \\
\hline$x^{2 w}$ & $0.973(0.039)$ & $0.603(0.039)$ & 0.025 & -2.011 & -0.787 & $\left|\Delta x^{2 w}\right|$ & $0.325(0.039)$ & $0.211(0.039)$ & 0.471 & 1.142 & 0.568 \\
\hline$x^{1 m}$ & $1.019(0.039)$ & $0.691(0.039)$ & 0.055 & -2.286 & -1.182 & $\left|\Delta x^{1 m}\right|$ & $0.366(0.039)$ & $0.243(0.039)$ & 0.476 & 0.755 & 0.550 \\
\hline$x^{2 m}$ & $1.082(0.039)$ & $0.688(0.039)$ & 0.051 & -3.826 & -2.103 & $\left|\Delta x^{2 m}\right|$ & $0.394(0.039)$ & $0.236(0.039)$ & 0.502 & -0.738 & -0.010 \\
\hline$x^{3 m}$ & $1.098(0.039)$ & $0.689(0.039)$ & 0.051 & -3.238 & -1.751 & $\Delta x^{3 m}$ & $0.409(0.039)$ & $0.257(0.039)$ & 0.491 & -1.318 & -0.222 \\
\hline$x^{4 m}$ & $1.074(0.039)$ & $0.668(0.039)$ & 0.067 & -2.808 & -2.019 & $\Delta x^{4 m}$ & $0.390(0.039)$ & $0.232(0.039)$ & 0.686 & -1.569 & -0.461 \\
\hline$x^{5 m}$ & $1.055(0.039)$ & $0.643(0.039)$ & 0.078 & -2.473 & -1.903 & $\left|\Delta x^{5 m}\right|$ & $0.377(0.039)$ & $0.201(0.039)$ & 1.080 & -1.602 & -0.533 \\
\hline$x^{6 m}$ & $1.017(0.039)$ & $0.592(0.039)$ & 0.107 & -2.218 & -1.858 & $\left|\Delta x^{6 m}\right|$ & $0.364(0.039)$ & $0.181(0.039)$ & 1.418 & -1.658 & -0.648 \\
\hline$x^{7 m}$ & $1.002(0.039)$ & $0.575(0.039)$ & 0.116 & -2.147 & -1.898 & $\Delta x^{7 m}$ & $0.341(0.039)$ & $0.164(0.039)$ & 1.728 & -1.057 & -0.392 \\
\hline$x^{8 m}$ & $0.983(0.039)$ & $0.550(0.039)$ & 0.127 & -2.147 & -1.966 & $\left|\Delta x^{8 m}\right|$ & $0.331(0.039)$ & $0.146(0.039)$ & 2.065 & -1.225 & -0.540 \\
\hline$x^{9 m}$ & $0.958(0.039)$ & $0.505(0.039)$ & 0.174 & -1.904 & -1.697 & $\left|\Delta x^{9 m}\right|$ & $0.333(0.039)$ & $0.162(0.039)$ & 2.203 & -0.384 & -0.106 \\
\hline$x^{10 m}$ & $0.944(0.039)$ & $0.503(0.039)$ & 0.166 & -2.008 & -1.865 & $\Delta x^{10 m} \mid$ & $0.331(0.039)$ & $0.158(0.039)$ & 2.511 & -0.644 & -0.308 \\
\hline$x^{11 m}$ & $0.928(0.039)$ & $0.484(0.039)$ & 0.202 & -1.955 & -1.834 & $\left|\Delta x^{11 m}\right|$ & $0.319(0.039)$ & $0.150(0.039)$ & 2.566 & -0.105 & 0.015 \\
\hline$x^{12 m}$ & $0.908(0.039)$ & $0.461(0.039)$ & 0.241 & -1.927 & -1.778 & $\left|\Delta x^{12 m}\right|$ & $0.325(0.039)$ & $0.159(0.039)$ & 3.034 & 0.486 & 0.434 \\
\hline mean & $0.995(0.039)$ & $0.584(0.039)$ & & & & mean & $0.351(0.039)$ & $0.191(0.039)$ & & & \\
\hline$W_{f}$ & $37.155(0.0004)$ & $56.375(0.0000)$ & & & & $W_{f}$ & $8.498(0.8097)$ & $12.223(0.5094)$ & & & \\
\hline
\end{tabular}

In the Table the estimated fractional differencing parameter, obtained using the Moulines and Soulier (1999) broad band log periodogram estimator (BBLP), is reported for the OIS spreads series in

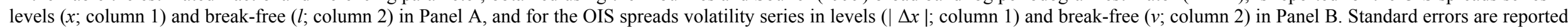

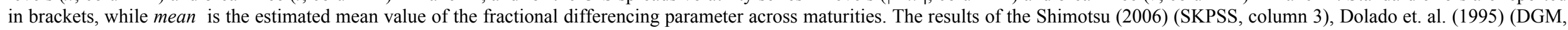
column 4), and Demetrescu et al. (2006) (DKH, column 5) long memory tests are also reported for the OIS spreads levels (Panel A) and volatilities (Panel B). The tabulated critical values at the 1\%, $5 \%$ and $10 \%$ significance level are $-4.866,-4.332,-3.924$, respectively, for the DGM test; $0.032,0.021,0.016$, respectively, for the SKPSS test; $-2.575,-1.96,-1.645$, respectively, for the DKH test. Finally, the Wald test for the null hypothesis of equal fractional differencing parameter across maturities $\left(W_{f}\right)$ is reported in the last row of the Table, with p-value in brackets. The results are for the various OIS spreads maturities available, i.e., from 1-week $\left(x^{1 w}\right)$ to one-year $\left(x^{12 m}\right)$. 
Table 3: Fractional differencing parameter subsample estimates and constancy tests: OIS spreads levels

\begin{tabular}{|c|c|c|c|c|c|c|c|c|c|c|c|c|}
\hline & \multicolumn{4}{|c|}{ BBLP-subsamples } & \multicolumn{2}{|c|}{ Equality tests across subsamples } & \multicolumn{6}{|c|}{ HM persistence break test } \\
\hline & Pre-crisis & Crisis+Post-crisis & Crisis & Post-crisis & $W_{i, 2}$ & $W_{i, 3}$ & $H M_{b}$ & $H M_{\max , b}$ & Date & $H M_{e}$ & $H M_{\max , e}$ & Date \\
\hline$x^{1 w}$ & $0.396(0.055)$ & $0.705(0.056)$ & $0.413(0.109)$ & $0.739(0.066)$ & $15.393(0.0001)$ & $18.045(0.0001)$ & 5.744 & 5.818 & $9 / 12 / 2007$ & 8.811 & 8.869 & $7 / 2 / 2008$ \\
\hline$x^{2 w}$ & $0.529(0.055)$ & $0.754(0.056)$ & $0.543(0.109)$ & $0.904(0.066)$ & $8.108(0.0044)$ & $21.335(0.0000)$ & 5.158 & 5.187 & $9 / 12 / 2007$ & 8.937 & 9.286 & $7 / 2 / 2008$ \\
\hline$x^{1 m}$ & $0.658(0.055)$ & $0.854(0.056)$ & $0.650(0.109)$ & $0.936(0.066)$ & $6.231(0.0126)$ & $14.576(0.0007)$ & 8.661 & 8.690 & $9 / 12 / 2007$ & 7.549 & 9.343 & $12 / 17 / 2008$ \\
\hline$x^{2 m}$ & $0.661(0.055)$ & $0.787(0.056)$ & $0.801(0.109)$ & $1.009(0.066)$ & $2.550(0.1103)$ & $13.984(0.0009)$ & 14.642 & 14.862 & $9 / 12 / 2007$ & 13.137 & 16.398 & $12 / 17 / 2008$ \\
\hline$x^{3 m}$ & $0.627(0.055)$ & $0.837(0.056)$ & $0.816(0.109)$ & $1.069(0.066)$ & $7.070(0.0078)$ & $24.848(0.0000)$ & 24.099 & 24.505 & $9 / 12 / 2007$ & 20.248 & 26.707 & $12 / 17 / 2008$ \\
\hline$x^{4 m}$ & $0.588(0.055)$ & $0.744(0.056)$ & $0.717(0.109)$ & $1.016(0.066)$ & $3.902(0.0482)$ & $22.044(0.0000)$ & 48.212 & 48.311 & $9 / 12 / 2007$ & 32.430 & 52.644 & $12 / 17 / 2008$ \\
\hline$x^{5 m}$ & $0.535(0.055)$ & $0.762(0.056)$ & $0.671(0.109)$ & $1.035(0.066)$ & $8.308(0.0039)$ & $30.397(0.0000)$ & 68.586 & 69.536 & $6 / 12 / 2007$ & 48.445 & 71.116 & $12 / 17 / 2008$ \\
\hline$x^{6 m}$ & $0.482(0.055)$ & $0.734(0.056)$ & $0.600(0.109)$ & $0.973(0.066)$ & $10.1960 .0014)$ & $31.525(0.0000)$ & 85.460 & 88.589 & $6 / 12 / 2007$ & 59.277 & 84.822 & $12 / 17 / 2008$ \\
\hline$x^{7 m}$ & $0.437(0.055)$ & $0.702(0.056)$ & $0.579(0.109)$ & $0.961(0.066)$ & $11.278(0.0008)$ & $36.911(0.0000)$ & 95.827 & 99.125 & $6 / 12 / 2007$ & 67.510 & 97.558 & $12 / 17 / 2008$ \\
\hline$x^{8 m}$ & $0.398(0.055)$ & $0.677(0.056)$ & $0.552(0.109)$ & $0.953(0.066)$ & $12.521(0.0004)$ & $39.628(0.0000)$ & 110.558 & 114.791 & $6 / 12 / 2007$ & 75.426 & 110.940 & $12 / 17 / 2008$ \\
\hline$x^{9 m}$ & $0.364(0.055)$ & $0.656(0.056)$ & $0.542(0.109)$ & $0.913(0.066)$ & $13.761(0.0002)$ & $40.290(0.0000)$ & 126.253 & 131.522 & $6 / 12 / 2007$ & 88.581 & 122.541 & $12 / 17 / 2008$ \\
\hline$x^{10 m}$ & $0.351(0.055)$ & $0.636(0.056)$ & $0.510(0.109)$ & $0.893(0.066)$ & $13.081(0.0003)$ & $40.227(0.0000)$ & 116.760 & 120.323 & $6 / 12 / 2007$ & 84.513 & 117.137 & $12 / 17 / 2008$ \\
\hline$x^{11 m}$ & $0.327(0.055)$ & $0.621(0.056)$ & $0.477(0.109)$ & $0.881(0.066)$ & $13.987(0.0002)$ & $41.943(0.0000)$ & 122.487 & 127.610 & $6 / 12 / 2007$ & 87.733 & 121.338 & $12 / 17 / 2008$ \\
\hline$x^{12 m}$ & $0.307(0.055)$ & $0.604(0.056)$ & $0.451(0.109)$ & $0.866(0.066)$ & $14.216(0.0002)$ & $44.091(0.0000)$ & 129.910 & 134.434 & $6 / 12 / 2007$ & 102.408 & 129.304 & $12 / 17 / 2008$ \\
\hline mean & $0.476(0.055)$ & $0.720(0.056)$ & $0.545(0.109)$ & $0.929(0.066)$ & & & & & & & & \\
\hline & $\begin{array}{c}W_{p r e:}: 66.945 \\
(0.0000)\end{array}$ & $\begin{array}{c}W_{c / p}: 24.040 \\
(\mathbf{0 . 0 4 5 3})\end{array}$ & $\begin{array}{c}W_{c}: 16.162 \\
(0.3036)\end{array}$ & $\begin{array}{c}W_{\text {post }}: 16.487 \\
(0.2845)\end{array}$ & $\begin{array}{c}W_{j, 2}: 140.602 \\
(0.0000)\end{array}$ & $\begin{array}{c}W_{j, 3}: 419.844 \\
(0.0000)\end{array}$ & & & & & & \\
\hline
\end{tabular}

In the Table the estimated fractional differencing parameter, obtained using the Moulines and Soulier (1999) broad band log periodogram estimator (BBLP), with standard error in brackets, is

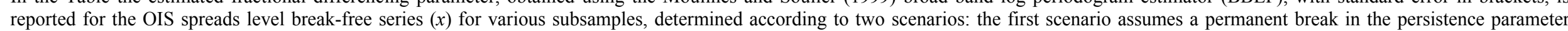
occurring after August 8 2007; the second scenario, in addition to the previous break, allows for a permanent change occurring after December 82008 . The pre-crisis sample therefore corresponds to the period May 62002 through August 72007 and the crisis/post-crisis sample to the period August 82007 through August 3 2012, for the first scenario; in addition to the pre-crisis sample, the crisis sample refers to the period August 82007 through December 82008 and the post-crisis sample to the period December 92008 through August 32012 , for the second scenario. The Wald test for the null hypothesis of equal fractional differencing parameter across the term structure $\left(W_{s} ; s=\right.$ pre, $c / p, c$, post $)$ and estimated mean values $($ mean $)$ for each subsample, as well as Wald tests for

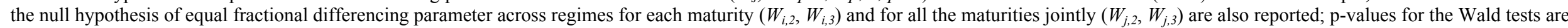
reported in brackets. Finally, the results of the Hassler and Meller (2009) test (HM) are reported with reference to the beginning $\left(H M_{b}\right)$ and the end $\left(H M_{e}\right)$ of the $c r i s i s$ period and for the two break points selected by the HM statistic $\left(H M_{\max , b}\right.$ and $\left.H M_{\max , e}\right)$; tabulated critical values are 5.398, 6.904 and 10.287 for the $10 \%, 5 \%$ and $1 \%$ significance level, respectively. The results are reported for the various OIS spreads maturities available, i.e., from 1-week $\left(x^{1 w}\right)$ to one-year ( $\left.x^{12 m}\right)$. 
Table 4: Fractional differencing parameter subsample estimates and constancy tests: OIS spreads volatilities

\begin{tabular}{|c|c|c|c|c|c|c|c|c|c|c|c|c|}
\hline & \multicolumn{4}{|c|}{ BBLP-subsamples } & \multicolumn{2}{|c|}{ Equality tests across subsamples } & \multicolumn{6}{|c|}{ HM persistence break test } \\
\hline & Pre-crisis & $\begin{array}{c}\text { Crisis+Post- } \\
\text { crisis }\end{array}$ & Crisis & Post-crisis & $W_{i, 2}$ & $W_{i, 3}$ & $H M_{b}$ & $H M_{\max , b}$ & Date & $H M_{e}$ & $H M_{\max , e}$ & Date \\
\hline$\left|\Delta x^{1 w}\right|$ & $0.231(0.055)$ & $0.128(0.056)$ & $0.318(0.109)$ & $0.250(0.066)$ & $1.717(0.4237)$ & $0.504(0.9180)$ & 0.377 & 0.604 & $5 / 18 / 2007$ & 2.382 & 2.256 & $1 / 22 / 2009$ \\
\hline$\left|\Delta x^{2 w}\right|$ & $0.197(0.055)$ & $0.171(0.056)$ & $0.304(0.109)$ & $0.236(0.066)$ & $0.111(0.9461)$ & $0.811(0.8468)$ & 0.119 & 0.081 & $5 / 18 / 2007$ & 4.525 & 6.422 & $1 / 22 / 2009$ \\
\hline$\Delta x^{1 m}$ & $0.221(0.055)$ & $0.256(0.056)$ & $0.272(0.109)$ & $0.326(0.066)$ & $0.195(0.9073)$ & $1.500(0.6823)$ & 0.091 & 0.046 & $5 / 18 / 2007$ & 2.598 & 3.198 & $1 / 22 / 2009$ \\
\hline$\left|\Delta x^{2 m}\right|$ & $0.239(0.055)$ & $0.255(0.056)$ & $0.414(0.109)$ & $0.198(0.066)$ & $0.040(0.9801)$ & $2.912(0.4053)$ & 0.339 & 0.002 & $5 / 18 / 2007$ & 0.372 & 6.057 & $8 / 6 / 2009$ \\
\hline$\left|\Delta x^{3 m}\right|$ & $0.248(0.055)$ & $0.288(0.056)$ & $0.446(0.109)$ & $0.224(0.066)$ & $0.258(0.8789)$ & $3.220(0.3589)$ & 0.239 & 0.082 & $5 / 18 / 2007$ & 0.288 & 4.683 & $8 / 6 / 2009$ \\
\hline$\left|\Delta x^{4 m}\right|$ & $0.232(0.055)$ & $0.265(0.056)$ & $0.415(0.109)$ & $0.151(0.066)$ & $0.177(0.9153)$ & $4.289(0.2319)$ & 0.319 & 0.022 & $5 / 18 / 2007$ & 1.397 & 4.609 & 8/6/2009 \\
\hline$\Delta x^{5 m}$ & $0.257(0.055)$ & $0.187(0.056)$ & $0.372(0.109)$ & $0.245(0.066)$ & $0.782(0.6763)$ & $1.078(0.7823)$ & 0.207 & 0.256 & $5 / 18 / 2007$ & 0.330 & 2.303 & $8 / 6 / 2009$ \\
\hline$\Delta x^{6 m}$ & $0.265(0.055)$ & $0.152(0.056)$ & $0.337(0.109)$ & $0.250(0.066)$ & $2.068(0.3555)$ & $0.470(0.9254)$ & 0.329 & 0.651 & $5 / 18 / 2007$ & 0.382 & 0.978 & $8 / 6 / 2009$ \\
\hline$\left|\Delta x^{7 m}\right|$ & $0.250(0.055)$ & $0.151(0.056)$ & $0.287(0.109)$ & $0.238(0.066)$ & $1.594(0.4506)$ & $0.149(0.9854)$ & 0.189 & 0.319 & $5 / 18 / 2007$ & 0.365 & 0.040 & 8/6/2009 \\
\hline$\Delta x^{8 m}$ & $0.237(0.055)$ & $0.125(0.056)$ & $0.266(0.109)$ & $0.275(0.066)$ & $2.025(0.3632)$ & $0.200(0.9776)$ & 0.230 & 0.387 & $5 / 18 / 2007$ & 0.291 & 0.551 & $8 / 6 / 2009$ \\
\hline$\Delta x^{9 m}$ & $0.236(0.055)$ & $0.142(0.056)$ & $0.221(0.109)$ & $0.281(0.066)$ & $1.429(0.4895)$ & $0.349(0.9506)$ & 0.711 & 1.049 & $5 / 18 / 2007$ & 0.498 & 0.940 & $8 / 6 / 2009$ \\
\hline$\left|\Delta x^{10 m}\right|$ & $0.219(0.055)$ & $0.154(0.056)$ & $0.199(0.109)$ & $0.284(0.066)$ & $0.683(0.7108)$ & $0.730(0.8661)$ & 0.426 & 0.707 & $5 / 18 / 2007$ & 0.439 & 1.911 & $8 / 6 / 2009$ \\
\hline$\left|\Delta x^{11 m}\right|$ & $0.212(0.055)$ & $0.128(0.056)$ & $0.214(0.109)$ & $0.249(0.066)$ & $1.150(0.5626)$ & $0.194(0.9786)$ & 0.650 & 1.042 & $5 / 18 / 2007$ & 0.431 & 1.608 & $8 / 6 / 2009$ \\
\hline$\Delta x^{12 m}$ & $0.209(0.055)$ & $0.144(0.056)$ & $0.220(0.109)$ & $0.274(0.066)$ & $0.692(0.7074)$ & $0.583(0.9004)$ & 0.411 & 0.617 & $5 / 18 / 2007$ & 0.590 & 2.040 & $8 / 6 / 2009$ \\
\hline Mean & $0.476(0.055)$ & $0.720(0.056)$ & $0.545(0.109)$ & $0.929(0.066)$ & & & & & & & & \\
\hline & $\begin{array}{c}W_{\text {pre }}: 1.584 \\
(0.9999)\end{array}$ & $\begin{array}{c}W_{c / p}: 7.216 \\
(0.9261)\end{array}$ & $\begin{array}{c}W_{c}: 5.201 \\
(0.9828)\end{array}$ & $\begin{array}{c}W_{\text {post }}: 16.487 \\
(0.2845)\end{array}$ & $\begin{array}{c}W_{j, 2}: 12.922 \\
(0.5326)\end{array}$ & $\begin{array}{c}W_{j, 3}: 16.990 \\
(0.9488)\end{array}$ & & & & & & \\
\hline
\end{tabular}

In the Table the estimated fractional differencing parameter, obtained using the Moulines and Soulier (1999) broad band log periodogram estimator (BBLP), with standard error in brackets, is reported for the OIS spreads volatility break-free series $(|\Delta x|)$ for various subsamples, determined according to two scenarios: the first scenario assumes a permanent break in the persistence parameter occurring after August 8 2007; the second scenario, in addition to the previous break, allows for a permanent change occurring after December 8 2008. The pre-crisis sample therefore corresponds to the period May 62002 through August 72007 and the crisis/post-crisis sample to the period August 82007 through August 32012 , for the first scenario; in addition to the pre-crisis sample, the crisis sample refers to the period August 82007 through December 82008 and the post-crisis sample to the period December 92008 through August 32012 , for the second scenario. The

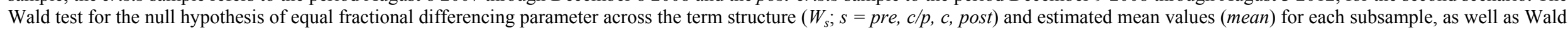
tests for the null hypothesis of equal fractional differencing parameter across regimes for each maturity $\left(W_{i, 2}, W_{i, 3}\right)$ and for all the maturities jointly $\left(W_{j, 2}, W_{j, 3}\right)$ are also reported; $\mathrm{p}$-values for the Wald tests are reported in brackets. Finally, the results of the Hassler and Meller (2009) test $(\mathrm{HM})$ are reported with reference to the beginning $\left(H M_{b}\right)$ and the end ( $\left.H M_{e}\right)$ of the $c r i s i s$ period and for the two

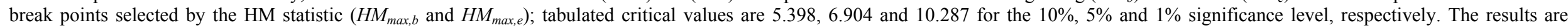
reported for the various OIS spreads maturities available, i.e., from 1-week ( $\left.x^{1 w}\right)$ to one-year $\left(x^{12 m}\right)$ 
Table 5: OIS spreads, principal components analysis, proportion of explained variance, total and for each series

\begin{tabular}{|c|c|c|c|c|c|c|c|c|c|c|c|c|c|c|c|c|c|c|c|}
\hline \multicolumn{20}{|c|}{ Panel A: Principal components analysis implemented on: } \\
\hline & \multicolumn{3}{|c|}{ OIS spreads $(x)$} & \multicolumn{3}{|c|}{ break processes (b) } & \multicolumn{3}{|c|}{ break-free OIS spreads (l) } & & \multicolumn{3}{|c|}{ OIS volatilities $(|\Delta \mathbf{x}|)$} & \multicolumn{3}{|c|}{ volatility break processes (c) } & \multicolumn{3}{|c|}{ break-free volatilities (v) } \\
\hline & $p c_{m 1}$ & $p c_{m 2}$ & $p c_{m 3}$ & $\mu_{m 1}$ & $\mu_{m 2}$ & $\mu_{m 3}$ & $f_{m 1}$ & $f_{m 2}$ & $f_{m 3}$ & & $p c_{v 1}$ & $p c_{v 2}$ & $p c_{v 3}$ & $\mu_{v 1}$ & $\mu_{v 2}$ & $\mu_{v 3}$ & $f_{v 1}$ & $f_{v 2}$ & $f_{v 3}$ \\
\hline tot & 0.949 & $\mathbf{0 . 0 4 3}$ & 0.007 & 0.954 & 0.036 & 0.008 & 0.792 & 0.117 & 0.045 & Tot & 0.762 & 0.150 & 0.046 & 0.959 & $\mathbf{0 . 0 3 7}$ & $\mathbf{0 . 0 0 2}$ & $\mathbf{0 . 6 2 4}$ & 0.231 & 0.071 \\
\hline$x^{1 w}$ & 0.434 & 0.427 & 0.118 & 0.458 & 0.416 & 0.111 & 0.321 & 0.493 & 0.055 & $\Delta x^{1 w} \mid$ & 0.353 & 0.532 & 0.065 & 0.759 & 0.232 & 0.002 & 0.333 & 0.556 & 0.056 \\
\hline$x^{2 w}$ & 0.514 & 0.415 & 0.062 & 0.510 & 0.400 & 0.086 & 0.375 & 0.528 & 0.026 & $\Delta x^{2 w} \mid$ & 0.389 & 0.488 & 0.011 & 0.691 & 0.296 & 0.007 & 0.396 & 0.474 & 0.009 \\
\hline$x^{1 m}$ & 0.596 & 0.363 & 0.002 & 0.578 & 0.373 & 0.026 & 0.522 & 0.300 & 0.001 & $\left|\Delta x^{1 m}\right|$ & 0.505 & 0.121 & 0.188 & 0.736 & 0.246 & 0.010 & 0.498 & 0.079 & 0.219 \\
\hline$x^{2 m}$ & 0.806 & 0.174 & 0.013 & 0.810 & 0.157 & 0.023 & 0.760 & 0.018 & 0.173 & $\left|\Delta x^{2 m}\right|$ & 0.645 & 0.066 & 0.235 & 0.833 & 0.124 & 0.030 & 0.672 & 0.025 & 0.241 \\
\hline$x^{3 m}$ & 0.887 & 0.092 & 0.018 & 0.886 & 0.083 & 0.028 & 0.841 & 0.004 & 0.123 & $\left|\Delta x^{3 m}\right|$ & 0.694 & 0.034 & 0.214 & 0.878 & 0.087 & 0.024 & 0.714 & 0.008 & 0.213 \\
\hline$x^{4 m}$ & 0.951 & 0.032 & 0.016 & 0.949 & 0.031 & 0.019 & 0.918 & 0.000 & 0.053 & $\left|\Delta x^{4 m}\right|$ & 0.796 & 0.007 & 0.142 & 0.934 & 0.047 & 0.003 & 0.784 & 0.000 & 0.147 \\
\hline$x^{5 m}$ & 0.980 & 0.007 & 0.010 & 0.979 & 0.008 & 0.011 & 0.946 & 0.006 & 0.020 & $\left|\Delta x^{5 m}\right|$ & 0.866 & 0.001 & 0.077 & 0.972 & 0.005 & 0.021 & 0.844 & 0.015 & 0.073 \\
\hline$x^{6 m}$ & 0.991 & 0.000 & 0.006 & 0.991 & 0.000 & 0.006 & 0.957 & 0.015 & 0.002 & $\left|\Delta x^{6 m}\right|$ & 0.893 & 0.019 & 0.025 & 0.980 & 0.000 & 0.016 & 0.849 & 0.051 & 0.019 \\
\hline$x^{7 m}$ & 0.994 & 0.003 & 0.002 & 0.996 & 0.001 & 0.002 & 0.942 & 0.029 & 0.000 & $\left|\Delta x^{7 m}\right|$ & 0.894 & 0.047 & 0.003 & 0.993 & 0.004 & 0.001 & 0.833 & 0.091 & 0.001 \\
\hline$x^{8 m}$ & 0.989 & 0.010 & 0.000 & 0.992 & 0.007 & 0.000 & 0.937 & 0.039 & 0.007 & $\left|\Delta x^{8 m}\right|$ & 0.891 & 0.069 & 0.001 & 0.978 & 0.013 & 0.005 & 0.819 & 0.121 & 0.005 \\
\hline$x^{9 m}$ & 0.977 & 0.022 & 0.001 & 0.981 & 0.018 & 0.001 & 0.925 & 0.041 & 0.023 & $\left|\Delta x^{9 m}\right|$ & 0.865 & 0.099 & 0.009 & 0.965 & 0.032 & 0.001 & 0.778 & 0.159 & 0.022 \\
\hline$x^{10 m}$ & 0.963 & 0.035 & 0.002 & 0.967 & 0.030 & 0.003 & 0.904 & 0.048 & 0.036 & $\Delta x^{10 m}$ & 0.845 & 0.113 & 0.020 & 0.957 & 0.037 & 0.004 & 0.750 & 0.176 & 0.041 \\
\hline$x^{11 m}$ & 0.944 & 0.050 & 0.006 & 0.949 & 0.044 & 0.006 & 0.885 & 0.054 & 0.048 & $\left|\Delta x^{11 m}\right|$ & 0.824 & 0.121 & 0.032 & 0.948 & 0.050 & 0.002 & 0.722 & 0.181 & 0.061 \\
\hline$x^{12 m}$ & 0.922 & 0.067 & 0.010 & 0.927 & 0.061 & 0.011 & 0.850 & 0.061 & 0.066 & $\Delta x^{12 m}$ & 0.799 & 0.127 & 0.041 & 0.929 & 0.068 & 0.003 & 0.690 & 0.186 & 0.072 \\
\hline
\end{tabular}

The Table reports the results of principal components analysis implemented on OIS spreads levels $(x)$ and volatilities $(|\Delta x|)$, their estimated break processes $(b, c)$ and break-free $(l, v)$ components.

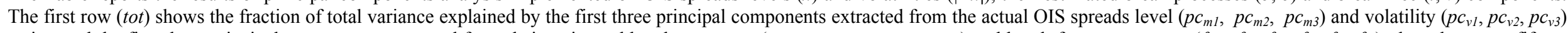
series, and the first three principal components extracted from their estimated break processes $\left(\mu_{m 1}, \mu_{m 2}, \mu_{m 3} ; \mu_{v 1}, \mu_{v 2}, \mu_{v 3}\right)$ and break-free components $\left(f_{m 1}, f_{m 2}, f_{m 3} ; f_{v 1}, f_{v 2}, f_{v 3}\right)$; the subsequent fifteen rows display the fraction of the variance of each individual series attributable to the extracted principal components for each set of series (actual, break, and break-free processes). Results are for the various OIS spreads maturities available, i.e., from 1-week $\left(x^{1 w}\right)$ to one-year $\left(x^{12 m}\right)$. 
Table 6: OIS spreads, principal components analysis, estimated loadings

Panel A: Principal components analysis implemented on:

\begin{tabular}{|c|c|c|c|c|c|c|c|c|c|c|c|c|c|c|c|c|c|c|c|}
\hline \multicolumn{20}{|c|}{ Panel A: Principal components analysis implemented on: } \\
\hline & \multicolumn{3}{|c|}{ OIS spreads $(x)$} & \multicolumn{3}{|c|}{ break processes (b) } & \multicolumn{3}{|c|}{ break-free OIS spreads (l) } & & \multicolumn{3}{|c|}{ OIS volatilities $(|\Delta \mathbf{x}|)$} & \multicolumn{3}{|c|}{ volatility break processes (c) } & \multicolumn{3}{|c|}{ break-free volatilities (v) } \\
\hline & $p c_{m 1}$ & $p c_{m 2}$ & $p c_{m 3}$ & $\mu_{m 1}$ & $\mu_{m 2}$ & $\mu_{m 3}$ & $f_{m 1}$ & $f_{m 2}$ & $f_{m 3}$ & & $p c_{v 1}$ & $p c_{v 2}$ & $p c_{v 3}$ & $\mu_{v 1}$ & $\mu_{v 2}$ & $\mu_{v 3}$ & $f_{v 1}$ & $f_{v 2}$ & $f_{v 3}$ \\
\hline & & & & & & & & & & & & & & & & & & & \\
\hline$x^{1 w}$ & 0.235 & -0.175 & 0.093 & 0.026 & -0.010 & 0.001 & 0.608 & -0.649 & 0.310 & $\left|\Delta x^{1 w}\right|$ & 0.039 & -0.040 & 0.014 & 0.026 & -0.010 & 0.001 & 0.030 & -0.038 & 0.012 \\
\hline$x^{2 w}$ & 0.264 & -0.178 & 0.070 & 0.023 & -0.010 & 0.001 & 0.663 & -0.681 & 0.172 & $\left|\Delta x^{2 w}\right|$ & 0.036 & -0.034 & 0.005 & 0.023 & -0.010 & 0.001 & 0.029 & -0.032 & 0.004 \\
\hline$x^{1 m}$ & 0.309 & -0.181 & 0.013 & 0.018 & -0.007 & 0.001 & 0.752 & -0.510 & -0.058 & $\left|\Delta x^{1 m}\right|$ & 0.028 & -0.012 & -0.014 & 0.018 & -0.007 & 0.001 & 0.022 & -0.009 & -0.014 \\
\hline$x^{2 m}$ & 0.413 & -0.144 & -0.039 & 0.017 & -0.004 & -0.002 & 0.875 & -0.178 & -0.393 & $\left|\Delta x^{2 m}\right|$ & 0.028 & -0.007 & -0.014 & 0.017 & -0.004 & -0.002 & 0.022 & -0.004 & -0.013 \\
\hline$x^{3 m}$ & 0.480 & -0.116 & -0.051 & 0.018 & -0.004 & -0.002 & 0.922 & -0.100 & -0.322 & $\left|\Delta x^{3 m}\right|$ & 0.029 & -0.005 & -0.013 & 0.018 & -0.004 & -0.002 & 0.023 & -0.002 & -0.013 \\
\hline$x^{4 m}$ & 0.527 & -0.073 & -0.051 & 0.021 & -0.003 & 0.001 & 0.954 & -0.015 & -0.208 & $\left|\Delta x^{4 m}\right|$ & 0.031 & -0.002 & -0.011 & 0.021 & -0.003 & 0.001 & 0.023 & 0.000 & -0.010 \\
\hline$x^{5 m}$ & 0.567 & -0.036 & -0.044 & 0.022 & -0.001 & -0.002 & 0.975 & 0.067 & -0.129 & $\left|\Delta x^{5 m}\right|$ & 0.033 & 0.001 & -0.008 & 0.022 & -0.001 & -0.002 & 0.024 & 0.003 & -0.007 \\
\hline$x^{6 m}$ & 0.603 & -0.003 & -0.036 & 0.025 & 0.000 & -0.002 & 0.979 & 0.117 & -0.041 & $\left|\Delta x^{6 m}\right|$ & 0.036 & 0.004 & -0.005 & 0.025 & 0.000 & -0.002 & 0.025 & 0.006 & -0.004 \\
\hline$x^{7 m}$ & 0.619 & 0.023 & -0.020 & 0.027 & 0.001 & -0.001 & 0.970 & 0.176 & 0.009 & $\left|\Delta x^{7 m}\right|$ & 0.038 & 0.007 & -0.002 & 0.027 & 0.001 & -0.001 & 0.026 & 0.009 & -0.001 \\
\hline$x^{8 m}$ & 0.632 & 0.048 & -0.004 & 0.030 & 0.002 & -0.001 & 0.967 & 0.212 & 0.062 & $\left|\Delta x^{8 m}\right|$ & 0.041 & 0.009 & 0.001 & 0.030 & 0.002 & -0.001 & 0.027 & 0.010 & 0.002 \\
\hline$x^{9 m}$ & 0.643 & 0.072 & 0.011 & 0.032 & 0.004 & -0.001 & 0.961 & 0.220 & 0.133 & $\left|\Delta x^{9 m}\right|$ & 0.043 & 0.012 & 0.004 & 0.032 & 0.004 & -0.001 & 0.028 & 0.012 & 0.005 \\
\hline$x^{10 m}$ & 0.655 & 0.093 & 0.025 & 0.035 & 0.005 & 0.001 & 0.950 & 0.242 & 0.168 & $\left|\Delta x^{10 m}\right|$ & 0.045 & 0.014 & 0.006 & 0.035 & 0.005 & 0.001 & 0.029 & 0.014 & 0.007 \\
\hline$x^{11 m}$ & 0.665 & 0.114 & 0.039 & 0.037 & 0.006 & 0.001 & 0.940 & 0.257 & 0.192 & $\left|\Delta x^{11 m}\right|$ & 0.047 & 0.015 & 0.008 & 0.037 & 0.006 & 0.001 & 0.029 & 0.015 & 0.009 \\
\hline$x^{12 m}$ & 0.675 & 0.136 & 0.052 & 0.039 & 0.007 & 0.001 & 0.921 & 0.276 & 0.228 & $\left|\Delta x^{12 m}\right|$ & 0.050 & 0.017 & 0.009 & 0.039 & 0.007 & 0.001 & 0.030 & 0.016 & 0.010 \\
\hline
\end{tabular}

The Table reports the estimated factor loadings for the first three principal components extracted from the actual OIS spreads level $\left(x, p c_{m l}, p c_{m 2}, p c_{m 3}\right)$ and volatility $\left(|\Delta \mathrm{x}|, p c_{v l}, p c_{v 2}, p c_{v 3}\right)$ series,

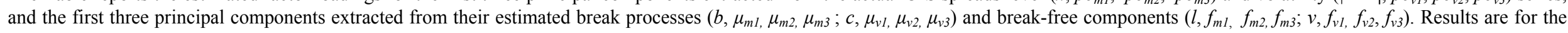
various OIS spreads maturities available, i.e., from 1-week $\left(x^{1 w}\right)$ to one-year $\left(x^{12 m}\right)$. 
Table 7:Fractional differencing parameter full and subsample estimates and constancy tests: OIS spreads break-free series levels and volatilities common factors

\begin{tabular}{|c|c|c|c|c|c|c|c|c|c|c|c|c|c|}
\hline \multirow[t]{2}{*}{ Level } & \multirow{2}{*}{$\begin{array}{c}\text { BBLP-full } \\
\text { sample }\end{array}$} & \multicolumn{4}{|c|}{ BBLP-subsamples } & \multicolumn{2}{|c|}{ Equality tests across subsamples } & \multicolumn{6}{|c|}{ HM persistence break test } \\
\hline & & Pre-crisis & $\begin{array}{l}\text { Crisis }+ \text { Post- } \\
\text { crisis }\end{array}$ & Crisis & Post-crisis & $W_{i, 2}$ & $W_{i, 3}$ & $H M_{b}$ & $H M_{\max , b}$ & Date & $H M_{e}$ & $H M_{\max , e}$ & Date \\
\hline$f_{m 1}$ & $\begin{array}{l}0.595 \\
(0.039)\end{array}$ & $\begin{array}{c}0.482 \\
(0.055)\end{array}$ & $\begin{array}{c}0.711 \\
(0.056)\end{array}$ & $\begin{array}{c}0.755 \\
(0.144)\end{array}$ & $\begin{array}{c}0.872 \\
(0.061)\end{array}$ & $\begin{array}{c}8.455 \\
(0.0146)\end{array}$ & $22.791(0.0000)$ & 72.791 & 75.119 & $8 / 17 / 2007$ & 48.229 & 75.922 & $9 / 8 / 2008$ \\
\hline$f_{m 2}$ & $\begin{array}{c}0.460 \\
(0.039)\end{array}$ & $\begin{array}{c}0.318 \\
(0.055)\end{array}$ & $\begin{array}{c}0.718 \\
(0.056)\end{array}$ & $\begin{array}{c}0.639 \\
(0.144)\end{array}$ & $\begin{array}{c}0.798 \\
(0.061)\end{array}$ & $\begin{array}{c}25.755 \\
(0.0000)\end{array}$ & $34.323(0.0000)$ & 14.061 & 14.385 & $10 / 5 / 2007$ & 24.635 & 24.388 & $9 / 4 / 2008$ \\
\hline$f_{m 2}$ & $\begin{array}{c}0.499 \\
(0.039)\end{array}$ & $\begin{array}{c}0.396 \\
(0.055)\end{array}$ & $\begin{array}{c}0.655 \\
(0.056)\end{array}$ & $\begin{array}{c}0.547 \\
(0.144)\end{array}$ & $\begin{array}{c}0.727 \\
(0.061)\end{array}$ & $\begin{array}{c}10.767 \\
(0.0046)\end{array}$ & $16.004(0.0000)$ & 28.025 & 28.043 & $8 / 20 / 2007$ & 41.012 & 44.194 & $9 / 4 / 2008$ \\
\hline Mean & $\begin{array}{c}0.528 \\
(0.039) \\
\end{array}$ & $\begin{array}{c}0.399 \\
(0.055) \\
\end{array}$ & $\begin{array}{c}0.695 \\
(0.056) \\
\end{array}$ & $\begin{array}{c}0.647 \\
(0.109) \\
\end{array}$ & $\begin{array}{c}0.799 \\
(0.066) \\
\end{array}$ & & & & & & & & \\
\hline$W_{f}$ & $\begin{array}{c}W_{f}: 6.150 \\
(0.0462)\end{array}$ & $\begin{array}{c}W_{p r e}: 4.444 \\
(0.2173)\end{array}$ & $\begin{array}{c}W_{c / p}: 0.753 \\
(\mathbf{0 . 8 6 0 6 )}\end{array}$ & $\begin{array}{c}W_{c}: 1.053 \\
(0.7886)\end{array}$ & $\begin{array}{c}W_{\text {post }}: \\
2.797 \\
(\mathbf{0 . 4 2 4 0 )}\end{array}$ & $\begin{array}{c}W_{j, 2}: 44.978 \\
(\mathbf{0 . 0 0 0 0 )}\end{array}$ & $\begin{array}{c}W_{j, 3}: 73.119 \\
(0.0000<)\end{array}$ & & & & & & \\
\hline
\end{tabular}

\begin{tabular}{|c|c|c|c|c|c|c|c|c|c|c|c|c|c|}
\hline \multirow[t]{2}{*}{ Volat } & \multirow[t]{2}{*}{$\begin{array}{c}\text { BBLP-full } \\
\text { sample } \\
\end{array}$} & \multicolumn{4}{|c|}{ BBLP-subsamples } & \multicolumn{2}{|c|}{ Equality tests across subsamples } & \multicolumn{6}{|c|}{ HM persistence break test } \\
\hline & & Pre-crisis & $\begin{array}{c}\text { Crisis }+ \text { Post- } \\
\text { crisis }\end{array}$ & Crisis & Post-crisis & $W_{i, 2}$ & $W_{i, 3}$ & $H M_{b}$ & $H M_{\max , b}$ & Date & $H M_{e}$ & $H M_{\text {max }, e}$ & Date \\
\hline$f_{v 1}$ & $\begin{array}{c}0.193 \\
(0.039) \\
\end{array}$ & $0.240(0.055)$ & $\begin{array}{l}0.182 \\
(0.056) \\
\end{array}$ & $\begin{array}{c}0.379 \\
(0.109) \\
\end{array}$ & $\begin{array}{c}0.268 \\
(0.066) \\
\end{array}$ & $\begin{array}{c}0.546 \\
(0.7609) \\
\end{array}$ & $1.283(0.7331)$ & 0.220 & 0.604 & $9 / 7 / 2007$ & 0.287 & 1.168 & $1 / 11 / 2008$ \\
\hline$f_{v 2}$ & $\begin{array}{c}0.175 \\
(0.039)\end{array}$ & $0.266(0.055)$ & $\begin{array}{c}0.145 \\
(0.056)\end{array}$ & $\begin{array}{c}0.250 \\
(0.109)\end{array}$ & $\begin{array}{c}0.283 \\
(0.066)\end{array}$ & $\begin{array}{c}2.354 \\
(0.3082)\end{array}$ & $0.080(0.9941)$ & 1.942 & 1.990 & $8 / 16 / 2007$ & 0.528 & 1.677 & $12 / 4 / 2007$ \\
\hline$f_{v 3}$ & $\begin{array}{c}0.119 \\
(0.039) \\
\end{array}$ & $0.190(0.055)$ & $\begin{array}{c}0.084 \\
(0.056)\end{array}$ & $\begin{array}{c}0.158 \\
(0.109) \\
\end{array}$ & $\begin{array}{c}0.253 \\
(0.066) \\
\end{array}$ & $\begin{array}{c}1.790 \\
(0.4086)\end{array}$ & $0.786(0.8529)$ & 0.317 & 0.488 & $12 / 8 / 2006$ & 0.891 & 2.359 & $5 / 6 / 2009$ \\
\hline Mean & $\begin{array}{c}0.162 \\
(0.039)\end{array}$ & $\begin{array}{c}0.232 \\
(0.055) \\
\end{array}$ & $\begin{array}{c}0.137 \\
(0.056) \\
\end{array}$ & $\begin{array}{c}0.262 \\
(0.109) \\
\end{array}$ & $\begin{array}{c}0.268 \\
(0.066)\end{array}$ & & & & & & & & \\
\hline$W_{f}$ & $\begin{array}{c}W_{f}: 1.918 \\
(0.3832)\end{array}$ & $\begin{array}{c}W_{\text {pre }}: 0.983 \\
(\mathbf{0 . 6 1 1 7})\end{array}$ & $\begin{array}{c}W_{c / p}: 1.523 \\
(0.6768)\end{array}$ & $\begin{array}{c}W_{c}: 16.162 \\
(0.3036)\end{array}$ & $\begin{array}{c}W_{\text {post }}: \\
16.487 \\
(\mathbf{0 . 2 8 4 5 )}\end{array}$ & $\begin{array}{c}W_{j, 2}: 4.690 \\
(0.1960)\end{array}$ & $\begin{array}{c}W_{j, 3}: 1.918 \\
(\mathbf{0 . 3 8 3 2})\end{array}$ & & & & & & \\
\hline
\end{tabular}

In the Table the estimated fractional differencing parameter obtained using the Moulines and Soulier (1999) broad band log periodogram estimator (BBLP) is reported for the first three principal

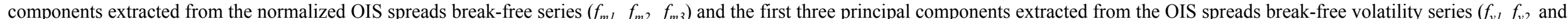

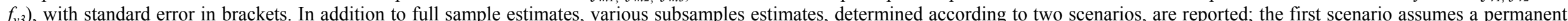

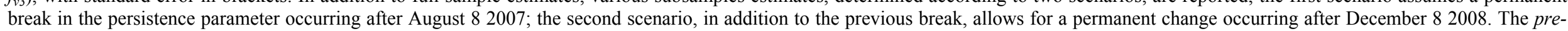

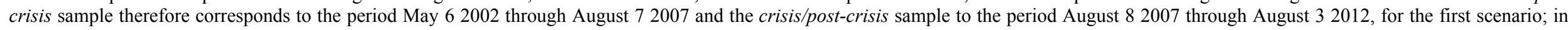
addition to the pre-crisis sample, the crisis sample refers to the period August 82007 through December 82008 and the post-crisis sample to the period December 92008 through August 32012 , for the second scenario. The Wald test for the null hypothesis of equal fractional differencing parameter across factors $(W \cdot s=f, p r e, c / p, c, p o s t)$ and estimated mean values $($ mean $)$ for the full sample and each subsample, as well as Wald tests for the null hypothesis of equal fractional differencing parameter across regimes for each factor $\left(W_{i, 2}, W_{i, 3}\right)$ and for all factors jointly $\left(W_{j, 2}, W_{j, 3}\right)$ are also

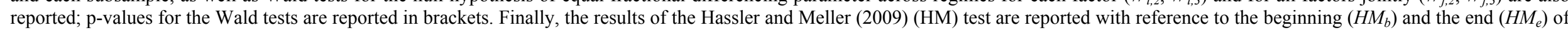

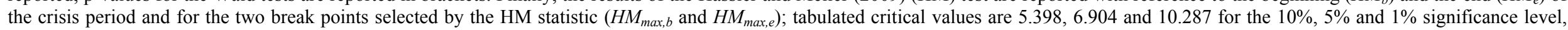
respectively. 
Table 8: OIS spreads, FI-H-FVAR estimates

\begin{tabular}{|c|c|c|c|c|c|c|c|c|c|c|c|c|c|c|c|c|}
\hline & \multicolumn{6}{|c|}{ Proportion of explained variance } & \multicolumn{6}{|c|}{ Factor loadings } & \multirow{2}{*}{\multicolumn{4}{|c|}{$\begin{array}{c}\text { Proportion of explained variance } \\
\text { Conditional variance processes }\end{array}$}} \\
\hline & \multicolumn{3}{|c|}{$\begin{array}{c}\text { break-free OIS spreads } \\
(x-b)\end{array}$} & \multicolumn{3}{|c|}{ break processes (b) } & \multicolumn{3}{|c|}{ Common long memory factors } & \multicolumn{3}{|c|}{ Common break processes } & & & & \\
\hline & $f_{m 1}$ & $f_{m 2}$ & $f_{m 3}$ & $\mu_{m 1}$ & $\mu_{m 2}$ & $\mu_{m 3}$ & $f_{m 1}$ & $f_{m 2}$ & $f_{m 3}$ & $\mu_{m 1}$ & $\mu_{m 2}$ & $\mu_{m 3}$ & & $g_{1}$ & $g_{2}$ & \\
\hline tot & 0.746 & 0.136 & 0.047 & 0.956 & 0.036 & 0.001 & & & & & & & tot & 0.993 & 0.007 & \\
\hline$x^{1 w}$ & 0.372 & 0.493 & 0.086 & 0.444 & 0.402 & 0.116 & $0.020(.006)$ & $-0.063(.009)$ & $0.000(.015)$ & $0.051(.002)$ & $-0.117(.010)$ & $0.081(.012)$ & & & & \\
\hline$x^{2 w}$ & 0.506 & 0.422 & 0.027 & 0.492 & 0.386 & 0.088 & $0.023(.006)$ & $-0.056(.009)$ & $0.013(.010)$ & $0.058(.002)$ & $-0.116(.007)$ & $0.049(.006)$ & & $h_{1}$ & $h_{2}$ & $h_{3}$ \\
\hline$x^{1 m}$ & 0.634 & 0.087 & 0.036 & 0.563 & 0.356 & 0.020 & $0.027(.005)$ & $-0.028(.009)$ & $0.051(.013)$ & $0.069(.001)$ & $-0.113(.003)$ & $-0.029(.022)$ & $g_{1}$ & 0.836 & 0.997 & 0.976 \\
\hline$x^{2 m}$ & 0.801 & 0.018 & 0.126 & 0.720 & 0.142 & 0.024 & $0.027(.003)$ & $-0.017(.005)$ & $0.017(.006)$ & $0.093(.001)$ & $-0.069(.004)$ & $-0.112(.014)$ & $g_{2}$ & 0.1636 & 0.001 & 0.002 \\
\hline$x^{3 m}$ & 0.863 & 0.005 & 0.091 & 0.806 & 0.076 & 0.026 & $0.029(.003)$ & $-0.014(.004)$ & $-0.003(.006)$ & $0.106(.001)$ & $-0.039(.004)$ & $-0.127(.008)$ & & Factor 1 & adings & \\
\hline$x^{4 m}$ & 0.908 & 0.001 & 0.037 & 0.894 & 0.029 & 0.017 & $0.029(.002)$ & $-0.006(.003)$ & $-0.021(.007)$ & $0.114(.001)$ & $-0.004(.003)$ & $-0.108(.008)$ & $\Lambda_{i, 1}$ & 2.511 & 3.262 & 4.017 \\
\hline$x^{5 m}$ & 0.899 & 0.015 & 0.009 & 0.924 & 0.007 & 0.009 & $0.028(.002)$ & $0.001(0.003)$ & $-0.027(.007)$ & $0.120(.001)$ & $0.023(.003)$ & $-0.081(.009)$ & $\Lambda_{i, 2}$ & -0.406 & 0.034 & 0.226 \\
\hline$x^{6 m}$ & 0.872 & 0.041 & 0.000 & 0.928 & 0.000 & 0.005 & $0.027(.002)$ & $0.007(.003)$ & $-0.028(.007)$ & $0.126(.001)$ & $0.048(.002)$ & $-0.058(.010)$ & & IGARCH & aramete & \\
\hline$x^{7 m}$ & 0.889 & 0.060 & 0.004 & 0.918 & 0.001 & 0.001 & $0.026(.002)$ & $0.011(.003)$ & $-0.020(.004)$ & $0.126(.001)$ & $0.064(.001)$ & $-0.019(.008)$ & $\phi$ & $\begin{array}{l}0.092 \\
(.103)\end{array}$ & $\begin{array}{c}0.000 \\
(.)\end{array}$ & $\begin{array}{c}0.000 \\
(.)\end{array}$ \\
\hline$x^{8 m}$ & 0.892 & 0.080 & 0.011 & 0.897 & 0.007 & 0.000 & $0.026(.002)$ & $0.013(.002)$ & $-0.011(.002)$ & $0.126(.001)$ & $0.079(.001)$ & $0.020(.006)$ & $\beta$ & $\begin{array}{l}0.292 \\
(.144)\end{array}$ & $\begin{array}{l}0.008 \\
(.030)\end{array}$ & $\begin{array}{l}0.076 \\
(.043)\end{array}$ \\
\hline$x^{9 m}$ & 0.865 & 0.101 & 0.024 & 0.869 & 0.016 & 0.001 & $0.025(.002)$ & $0.016(.003)$ & $-0.001(.003)$ & $0.126(.001)$ & $0.094(.003)$ & $0.055(.005)$ & $b$ & $\begin{array}{l}0.415 \\
(.044)\end{array}$ & $\begin{array}{l}0.395 \\
(.014)\end{array}$ & $\begin{array}{l}0.326 \\
(.024)\end{array}$ \\
\hline$x^{10 m}$ & 0.826 & 0.116 & 0.039 & 0.841 & 0.026 & 0.002 & $0.025(.002)$ & $0.019(.003)$ & $0.007(.004)$ & $0.126(.001)$ & $0.107(.004)$ & $0.087(.004)$ & & & & \\
\hline$x^{11 m}$ & 0.770 & 0.123 & 0.056 & 0.810 & 0.037 & 0.005 & $0.025(.002)$ & $0.021(.004)$ & $0.016(.007)$ & $0.125(.001)$ & $0.120(.005)$ & $0.117(.005)$ & $\mathrm{BIC}_{\mathrm{cv}}$ & 0.556 & -0.403 & -1.452 \\
\hline$x^{12 m}$ & 0.691 & 0.130 & 0.071 & 0.776 & 0.049 & 0.008 & $0.025(.002)$ & $0.023(.004)$ & $0.026(.009)$ & $0.124(.001)$ & $0.134(.006)$ & $0.150(.006)$ & $\mathrm{BIC}_{\mathrm{sys}}$ & -124.921 & & \\
\hline
\end{tabular}

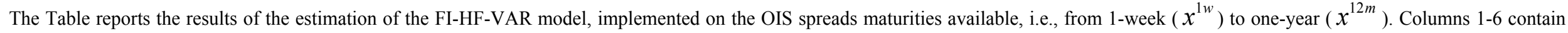

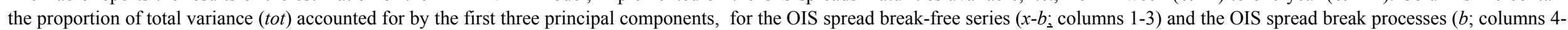
6). Columns 1-12 contain results for the conditional mean processes, while columns 13-16 contain results for the conditional variance processes. In columns 1-6 the first row (tot) shows the fraction of total variance explained by the first three principal components extracted from the OIS spreads break-free series $\left(f_{m,} f_{m 2} f_{m 3}\right)$ and estimated break processes $\left(\mu_{m}, \mu_{m 2} \mu_{m 3}\right)$; the subsequent fifteen

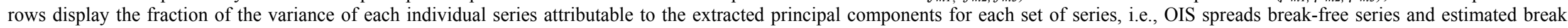

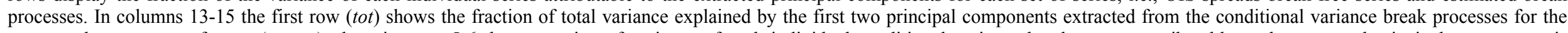
common long memory factors $\left(g_{l}, g_{2}\right)$; then, in rows 5-6 the proportion of variance of each individual conditional variance break process attributable to the extracted principal components is reported. Factor loadings for the common stochastic and deterministic factors in mean and variance are reported in columns 7-12 and 14-16 (rows 8-9), respectively. Parameters for the FIGARCH

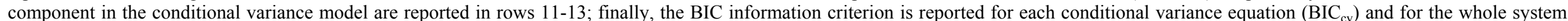
$\left(\mathrm{BIC}_{\mathrm{sys}}\right)$. Standard errors are reported in brackets. 
Table 9: FI-H-FVAR estimates; conditional correlations

Panel A: Pre-crisis versus crisis correlations

\begin{tabular}{|c|c|c|c|c|c|c|c|c|c|c|c|c|c|c|}
\hline \multicolumn{10}{|c|}{ Panel A: Pre-crisis versus crisis correlations } \\
\hline pre / crisis & $x^{1 w}$ & $x^{2 w}$ & $x^{1 m}$ & $x^{2 m}$ & $x^{3 m}$ & $x^{4 m}$ & $x^{5 m}$ & $x^{6 m}$ & $x^{7 m}$ & $x^{8 m}$ & $x^{9 m}$ & $x^{10 m}$ & $x^{11 m}$ & $x^{12 m}$ \\
\hline$x^{1 w}$ & & 0.988 & 0.756 & 0.772 & 0.738 & 0.595 & 0.476 & 0.365 & 0.310 & 0.259 & 0.201 & 0.156 & 0.122 & 0.081 \\
\hline$x^{2 w}$ & 0.986 & & 0.849 & 0.849 & 0.793 & 0.634 & 0.511 & 0.404 & 0.365 & 0.333 & 0.293 & 0.263 & 0.241 & 0.210 \\
\hline$x^{1 m}$ & 0.719 & 0.822 & & 0.944 & 0.819 & 0.631 & 0.521 & 0.447 & 0.473 & 0.510 & 0.542 & 0.567 & 0.592 & 0.604 \\
\hline$x^{2 m}$ & 0.746 & 0.829 & 0.930 & & 0.962 & 0.847 & 0.763 & 0.699 & 0.706 & 0.716 & 0.713 & 0.705 & 0.695 & 0.672 \\
\hline$x^{3 m}$ & 0.708 & 0.762 & 0.772 & 0.950 & & 0.957 & 0.901 & 0.847 & 0.836 & 0.819 & 0.784 & 0.747 & 0.707 & 0.655 \\
\hline$x^{4 m}$ & 0.542 & 0.572 & 0.538 & 0.807 & 0.948 & & 0.987 & 0.958 & 0.939 & 0.905 & 0.850 & 0.792 & 0.730 & 0.657 \\
\hline$x^{5 m}$ & 0.406 & 0.430 & 0.407 & 0.707 & 0.883 & 0.985 & & 0.991 & 0.975 & 0.941 & 0.884 & 0.823 & 0.755 & 0.678 \\
\hline$x^{6 m}$ & 0.281 & 0.308 & 0.323 & 0.633 & 0.822 & 0.955 & 0.991 & & 0.992 & 0.965 & 0.915 & 0.858 & 0.792 & 0.717 \\
\hline$x^{7 m}$ & 0.218 & 0.265 & 0.360 & 0.648 & 0.814 & 0.934 & 0.972 & 0.991 & & 0.990 & 0.958 & 0.914 & 0.860 & 0.795 \\
\hline$x^{8 m}$ & 0.161 & 0.230 & 0.413 & 0.667 & 0.798 & 0.895 & 0.931 & 0.957 & 0.988 & & 0.989 & 0.962 & 0.922 & 0.871 \\
\hline$x^{9 m}$ & 0.093 & 0.185 & 0.459 & 0.668 & 0.757 & 0.828 & 0.861 & 0.894 & 0.947 & 0.986 & & 0.992 & 0.969 & 0.934 \\
\hline$x^{10 m}$ & 0.040 & 0.151 & 0.495 & 0.660 & 0.711 & 0.754 & 0.783 & 0.820 & 0.890 & 0.950 & 0.989 & & 0.993 & 0.972 \\
\hline$x^{11 m}$ & 0.001 & 0.127 & 0.530 & 0.649 & 0.660 & 0.674 & 0.696 & 0.735 & 0.820 & 0.899 & 0.960 & 0.990 & & 0.993 \\
\hline$x^{12 m}$ & -0.043 & 0.095 & 0.548 & 0.621 & 0.594 & 0.582 & 0.598 & 0.639 & 0.737 & 0.833 & 0.914 & 0.963 & 0.991 & \\
\hline
\end{tabular}

\begin{tabular}{|c|c|c|c|c|c|c|c|c|c|c|c|c|c|c|}
\hline \multicolumn{15}{|c|}{ Panel B: Post-crisis versus crisis correlations } \\
\hline post / crisis & $x^{1 w}$ & $x^{2 w}$ & $x^{1 m}$ & $x^{2 m}$ & $x^{3 m}$ & $x^{4 m}$ & $x^{5 m}$ & $x^{6 m}$ & $x^{7 m}$ & $x^{8 m}$ & $x^{9 m}$ & $x^{10 m}$ & $x^{11 m}$ & $x^{12 m}$ \\
\hline$x^{1 w}$ & & 0.988 & 0.756 & 0.772 & 0.738 & 0.595 & 0.476 & 0.365 & 0.310 & 0.259 & 0.201 & 0.156 & 0.122 & 0.081 \\
\hline$x^{2 w}$ & 0.988 & & 0.849 & 0.849 & 0.793 & 0.634 & 0.511 & 0.404 & 0.365 & 0.333 & 0.293 & 0.263 & 0.241 & 0.210 \\
\hline$x^{1 m}$ & 0.729 & 0.825 & & 0.944 & 0.819 & 0.631 & 0.521 & 0.447 & 0.473 & 0.510 & 0.542 & 0.567 & 0.592 & 0.604 \\
\hline$x^{2 m}$ & 0.743 & 0.822 & 0.933 & & 0.962 & 0.847 & 0.763 & 0.699 & 0.706 & 0.716 & 0.713 & 0.705 & 0.695 & 0.672 \\
\hline$x^{3 m}$ & 0.699 & 0.753 & 0.781 & 0.952 & & 0.957 & 0.901 & 0.847 & 0.836 & 0.819 & 0.784 & 0.747 & 0.707 & 0.655 \\
\hline$x^{4 m}$ & 0.520 & 0.553 & 0.546 & 0.808 & 0.946 & & 0.987 & 0.958 & 0.939 & 0.905 & 0.850 & 0.792 & 0.730 & 0.657 \\
\hline$x^{5 m}$ & 0.370 & 0.399 & 0.407 & 0.701 & 0.875 & 0.983 & & 0.991 & 0.975 & 0.941 & 0.884 & 0.823 & 0.755 & 0.678 \\
\hline$x^{6 m}$ & 0.230 & 0.262 & 0.312 & 0.617 & 0.805 & 0.947 & 0.989 & & 0.992 & 0.965 & 0.915 & 0.858 & 0.792 & 0.717 \\
\hline$x^{7 m}$ & 0.158 & 0.208 & 0.338 & 0.622 & 0.788 & 0.921 & 0.968 & 0.990 & & 0.990 & 0.958 & 0.914 & 0.860 & 0.795 \\
\hline$x^{8 m}$ & 0.091 & 0.162 & 0.379 & 0.630 & 0.764 & 0.878 & 0.924 & 0.956 & 0.988 & & 0.989 & 0.962 & 0.922 & 0.871 \\
\hline$x^{9 m}$ & 0.016 & 0.107 & 0.411 & 0.621 & 0.716 & 0.806 & 0.852 & 0.893 & 0.947 & 0.986 & & 0.992 & 0.969 & 0.934 \\
\hline$x^{10 m}$ & -0.040 & 0.067 & 0.438 & 0.607 & 0.665 & 0.731 & 0.774 & 0.820 & 0.892 & 0.952 & 0.990 & & 0.993 & 0.972 \\
\hline$x^{11 m}$ & -0.080 & 0.040 & 0.467 & 0.592 & 0.614 & 0.653 & 0.689 & 0.738 & 0.824 & 0.903 & 0.962 & 0.991 & & 0.993 \\
\hline$x^{12 m}$ & -0.124 & 0.006 & 0.479 & 0.562 & 0.548 & 0.562 & 0.594 & 0.646 & 0.745 & 0.840 & 0.919 & 0.965 & 0.992 & \\
\hline
\end{tabular}

The Table reports the average conditional correlation coefficients computed over the pre-crisis sample (May 62002 through August 7 2007), the crisis sample (August 82007 through December 8 2008) and the post-crisis sample (December 92008 through August 3 2012). The upper triangular matrix, in both Panel A and B, contains results for the crisis sample; the lower triangular matrix in Panel A contains figures for the pre-crisis sample, while the lower triangular matrix in Panel B contains figures for the post-crisis sample. Figures in bolds are statistically different, at the 5\% significance level, across the regimes considered. The test for homogeneity of the correlation coefficients is based on the Fisher's Z-transform (Paul, 1989). The results are reported for the various OIS spreads maturities available, i.e., from 1-week $\left(x^{1 w}\right)$ to one-year $\left(x^{12 m}\right)$. 
Table 10: Out of sample forecasting analysis

\begin{tabular}{|c|c|c|c|c|c|c|c|c|c|c|c|c|c|c|}
\hline \multicolumn{15}{|c|}{ Panel A: Unemployment rate changes } \\
\hline Step & NAIVE & AR & B & B1 & B2 & $\mathrm{F}$ & F1 & F2 & F3 & A1 & A2 & A3 & $\mathrm{C}$ & $\mathrm{CF}$ \\
\hline \multicolumn{15}{|l|}{ COR } \\
\hline 1 & 0.449 & 0.411 & 0.426 & 0.422 & 0.412 & 0.586 & 0.515 & 0.549 & 0.475 & 0.614 & 0.602 & 0.580 & 0.670 & 0.632 \\
\hline 3 & 0.550 & 0.508 & 0.536 & 0.552 & 0.513 & 0.748 & 0.623 & 0.777 & 0.480 & 0.692 & 0.792 & 0.758 & 0.808 & 0.809 \\
\hline 6 & 0.540 & 0.401 & 0.531 & 0.499 & 0.411 & 0.729 & 0.519 & 0.793 & 0.344 & 0.619 & 0.732 & 0.732 & 0.755 & 0.859 \\
\hline 12 & 0.493 & 0.142 & 0.514 & 0.392 & 0.159 & 0.557 & 0.295 & 0.638 & 0.090 & 0.376 & 0.584 & 0.613 & 0.559 & 0.696 \\
\hline \multicolumn{15}{|c|}{ RMSFE } \\
\hline 1 & 0.239 & 0.213 & 0.207 & 0.207 & 0.211 & 0.185 & 0.193 & 0.196 & 0.206 & 0.178 & 0.180 & 0.200 & 0.167 & 0.177 \\
\hline 3 & 0.600 & 0.502 & 0.471 & 0.460 & 0.506 & 0.368 & 0.431 & 0.410 & 0.508 & 0.400 & 0.359 & 0.449 & 0.322 & 0.322 \\
\hline 6 & 1.189 & 1.032 & 0.925 & 0.900 & 1.036 & 0.818 & 0.911 & 0.763 & 1.011 & 0.880 & 0.684 & 0.892 & 0.726 & 0.570 \\
\hline 12 & 2.448 & 2.047 & 1.683 & 1.737 & 2.068 & 1.878 & 2.012 & 1.547 & 2.011 & 2.661 & 1.530 & 1.773 & 1.780 & 1.991 \\
\hline \multicolumn{15}{|l|}{$\mathbf{U}$} \\
\hline 1 & 0.492 & 0.509 & 0.504 & 0.508 & 0.507 & 0.445 & 0.463 & 0.476 & 0.489 & 0.411 & 0.446 & 0.478 & 0.407 & 0.414 \\
\hline 3 & 0.449 & 0.436 & 0.433 & 0.427 & 0.437 & 0.339 & 0.378 & 0.398 & 0.475 & 0.343 & 0.350 & 0.394 & 0.271 & 0.279 \\
\hline 6 & 0.461 & 0.479 & 0.450 & 0.446 & 0.479 & 0.370 & 0.421 & 0.377 & 0.505 & 0.386 & 0.354 & 0.418 & 0.307 & 0.246 \\
\hline 12 & 0.499 & 0.589 & 0.493 & 0.507 & 0.590 & 0.490 & 0.519 & 0.445 & 0.603 & 0.539 & 0.412 & 0.498 & 0.459 & 0.402 \\
\hline
\end{tabular}

\begin{tabular}{|c|c|c|c|c|c|c|c|c|c|c|c|c|c|c|}
\hline \multicolumn{15}{|c|}{ Panel B: Industrial production growth rate } \\
\hline Step & NAIVE & AR & $\mathrm{B}$ & $\mathrm{B} 1$ & B2 & $\mathrm{F}$ & F1 & F2 & F3 & A1 & $\mathrm{A} 2$ & A3 & $\mathrm{C}$ & $\mathrm{CF}$ \\
\hline \multicolumn{15}{|l|}{ COR } \\
\hline 1 & 0.441 & 0.458 & 0.486 & 0.483 & 0.402 & 0.589 & 0.463 & 0.625 & 0.490 & 0.467 & 0.588 & 0.592 & 0.555 & 0.582 \\
\hline 3 & 0.557 & 0.483 & 0.614 & 0.563 & 0.495 & 0.732 & 0.517 & 0.770 & 0.509 & 0.608 & 0.686 & 0.771 & 0.664 & 0.678 \\
\hline 6 & 0.569 & 0.311 & 0.534 & 0.434 & 0.336 & 0.671 & 0.405 & 0.706 & 0.325 & 0.483 & 0.569 & 0.675 & 0.547 & 0.667 \\
\hline 12 & 0.407 & 0.010 & 0.402 & 0.246 & 0.048 & 0.459 & 0.115 & 0.462 & -0.004 & 0.162 & 0.275 & 0.415 & 0.258 & 0.435 \\
\hline \multicolumn{15}{|c|}{ RMSFE } \\
\hline 1 & 1.163 & 0.985 & 0.994 & 0.965 & 1.021 & 0.917 & 0.994 & 0.895 & 1.009 & 1.009 & 0.930 & 0.968 & 0.916 & 0.928 \\
\hline 3 & 2.839 & 2.281 & 2.087 & 2.137 & 2.312 & 1.858 & 2.257 & 1.909 & 2.290 & 2.266 & 1.899 & 2.063 & 1.977 & 2.090 \\
\hline 6 & 5.546 & 4.768 & 4.324 & 4.354 & 4.730 & 4.022 & 4.771 & 3.857 & 4.758 & 5.261 & 3.944 & 4.228 & 4.272 & 4.827 \\
\hline 12 & 12.531 & 8.785 & 8.202 & 8.300 & 8.992 & 11.069 & 8.819 & 7.591 & 8.820 & 15.814 & 8.124 & 8.285 & 8.844 & 14.373 \\
\hline \multicolumn{15}{|l|}{$\mathbf{U}$} \\
\hline 1 & 0.521 & 0.558 & 0.523 & 0.551 & 0.559 & 0.463 & 0.533 & 0.463 & 0.508 & 0.527 & 0.509 & 0.513 & 0.491 & 0.461 \\
\hline 3 & 0.476 & 0.502 & 0.459 & 0.498 & 0.497 & 0.382 & 0.482 & 0.427 & 0.508 & 0.435 & 0.448 & 0.419 & 0.405 & 0.395 \\
\hline 6 & 0.480 & 0.577 & 0.508 & 0.540 & 0.567 & 0.414 & 0.536 & 0.447 & 0.595 & 0.504 & 0.497 & 0.473 & 0.472 & 0.429 \\
\hline 12 & 0.581 & 0.699 & 0.569 & 0.625 & 0.687 & 0.572 & 0.672 & 0.560 & 0.717 & 0.683 & 0.607 & 0.581 & 0.644 & 0.615 \\
\hline
\end{tabular}

\begin{tabular}{|c|c|c|c|c|c|c|c|c|c|c|c|c|c|c|}
\hline \multicolumn{15}{|c|}{ Panel C: Inflation rate } \\
\hline Step & NAIVE & $\mathrm{AR}$ & $\mathrm{B}$ & $\mathrm{B} 1$ & $\mathrm{~B} 2$ & $\mathrm{~F}$ & F1 & F2 & F3 & A1 & $\mathrm{A} 2$ & A3 & $\mathrm{C}$ & $\mathrm{CF}$ \\
\hline \multicolumn{15}{|l|}{ COR } \\
\hline 1 & 0.588 & 0.338 & 0.381 & 0.402 & 0.394 & 0.591 & 0.378 & 0.533 & 0.598 & 0.371 & 0.511 & 0.418 & 0.350 & 0.604 \\
\hline 3 & 0.336 & 0.162 & 0.111 & 0.103 & 0.180 & 0.120 & -0.032 & 0.175 & 0.146 & 0.091 & 0.071 & 0.203 & 0.009 & 0.097 \\
\hline 6 & 0.085 & 0.080 & -0.079 & -0.077 & 0.043 & -0.079 & -0.213 & -0.039 & 0.083 & -0.027 & -0.031 & 0.054 & -0.160 & -0.115 \\
\hline 12 & -0.130 & -0.135 & -0.409 & -0.368 & -0.312 & -0.247 & -0.111 & -0.426 & 0.071 & -0.116 & -0.218 & -0.188 & -0.248 & -0.202 \\
\hline & & & & & & & & & & & & & & \\
\hline \multicolumn{15}{|c|}{ RMSFE } \\
\hline 1 & 0.460 & 0.435 & 0.438 & 0.428 & 0.421 & 0.382 & 0.438 & 0.397 & 0.364 & 0.468 & 0.400 & 0.421 & 0.455 & 0.376 \\
\hline 3 & 1.548 & 1.077 & 1.104 & 1.093 & 1.072 & 1.137 & 1.197 & 1.157 & 1.119 & 1.254 & 1.143 & 1.142 & 1.256 & 1.230 \\
\hline 6 & 3.238 & 1.672 & 1.714 & 1.690 & 1.645 & 1.818 & 1.808 & 1.839 & 1.715 & 2.070 & 1.841 & 1.845 & 2.044 & 2.140 \\
\hline 12 & 6.243 & 2.067 & 2.087 & 2.079 & 2.035 & 2.463 & 2.198 & 2.346 & 2.101 & 3.323 & 2.431 & 2.418 & 2.616 & 3.571 \\
\hline \multicolumn{15}{|l|}{$\mathbf{U}$} \\
\hline 1 & 0.498 & 0.554 & 0.535 & 0.526 & 0.539 & 0.429 & 0.535 & 0.455 & 0.438 & 0.529 & 0.469 & 0.514 & 0.533 & 0.426 \\
\hline 3 & 0.616 & 0.559 & 0.577 & 0.585 & 0.568 & 0.592 & 0.641 & 0.545 & 0.565 & 0.590 & 0.566 & 0.537 & 0.633 & 0.607 \\
\hline 6 & 0.721 & 0.519 & 0.559 & 0.579 & 0.539 & 0.569 & 0.590 & 0.538 & 0.524 & 0.608 & 0.543 & 0.520 & 0.622 & 0.609 \\
\hline 12 & 0.817 & 0.415 & 0.469 & 0.467 & 0.440 & 0.504 & 0.461 & 0.451 & 0.424 & 0.600 & 0.461 & 0.445 & 0.526 & 0.616 \\
\hline
\end{tabular}

The Table reports the results of the out of sample forecasting analysis for the unemployment rate (in changes; Panel A), the industrial production growth rate (Panel B) and the CPI inflation rate (Panel C), at different horizons, i.e., 1-month, 3-month, 6-month and 12-month; the forecasting sample is from August 2007 through July 2012. The reported statistics are the simple correlation coefficient between actual and forecasted values (COR), the root mean square forecast error (RMSFE) and the Theil's $I C$ coefficient. Forecasts are generated from AR/VAR models with up to 5-lags; the best outcome for each forecasting indicator is then reported in the table for any horizon. In addition to the "no change" forecasting model (NAIVE) and the autoregressive model, including information about the own target variable only (AR), VAR models for the target variable and various indicators are employed, i.e., the B model, including the Federal funds rate and the term spread; the B1 model, including the Federal funds rate only; the B2 model, including the term spread only; the F model, including the estimated level, slope and curvature factor conditional means; the F1 model, including the estimated level factor conditional mean only; the F2 model, including the estimated slope factor conditional mean only; the F3 model, including the estimated curvature factor conditional mean only; the A1 model, including the corporate spread; the A2 model, including the TED spread; the A3 model, including the mortgage spread; the $\mathrm{C}$ model, including the composite indicator constructed from the common component in the estimated level factor and the TED, corporate and mortgage spreads (FRAG); the CF model, including FRAG and the estimated slope and curvature factor conditional means. 
Table 11: forecast error variance decomposition

\begin{tabular}{|c|c|c|c|c|c|c|c|c|c|c|c|c|c|c|c|c|c|c|c|c|c|c|}
\hline & & \multicolumn{7}{|c|}{$\begin{array}{c}\text { Pre-crisis sample } \\
\end{array}$} & \multicolumn{7}{|c|}{$\begin{array}{c}\text { Crisis sample } \\
\end{array}$} & \multicolumn{7}{|c|}{$\begin{array}{c}\text { Post-crisis sample } \\
\end{array}$} \\
\hline & \multirow[b]{2}{*}{ Horizon } & \multicolumn{4}{|c|}{ Common factors shocks } & \multicolumn{3}{|c|}{ Idiosyncratic shocks } & \multicolumn{4}{|c|}{ Common factors shocks } & \multicolumn{3}{|c|}{ Idiosyncratic shocks } & \multicolumn{4}{|c|}{ Common factors shocks } & \multicolumn{3}{|c|}{ Idiosyncratic shocks } \\
\hline & & $f_{1}$ & $f_{2}$ & $f_{3}$ & all & Own & other & all & $f_{1}$ & $f_{2}$ & $f_{3}$ & all & own & other & all & $f_{1}$ & $f_{2}$ & $f_{3}$ & all & own & other & all \\
\hline \multirow{2}{*}{$x^{1 w}$} & 1 & 16.9 & 44.5 & 11.6 & 73.0 & 27.0 & 0.0 & 27.0 & 22.6 & 45.4 & 10.4 & 78.5 & 21.5 & 0.0 & 21.5 & 29.3 & 48.0 & 8.8 & 86.0 & 14.0 & 0.0 & 14.0 \\
\hline & 20 & 18.6 & 42.1 & 10.9 & 71.5 & 28.5 & 0.0 & 28.5 & 29.2 & 55.2 & 6.2 & 90.6 & 9.4 & 0.0 & 9.4 & 32.0 & 59.7 & 5.3 & 97.1 & 2.9 & 0.0 & 2.9 \\
\hline \multirow{2}{*}{$x^{2 w}$} & 1 & 24.8 & 45.0 & 4.9 & 74.7 & 13.4 & 11.8 & 25.3 & 31.6 & 44.4 & 4.1 & 80.2 & 19.6 & 0.2 & 19.8 & 40.0 & 45.3 & 3.5 & 88.8 & 8.9 & 2.3 & 11.2 \\
\hline & 20 & 28.7 & 44.9 & 4.9 & 78.5 & 11.4 & 10.1 & 21.5 & 39.3 & 51.9 & 2.4 & 93.7 & 6.3 & 0.1 & 6.3 & 41.9 & 54.4 & 2.1 & 98.3 & 1.3 & 0.3 & 1.7 \\
\hline \multirow{2}{*}{$x^{1 m}$} & 1 & 38.8 & 32.8 & 2.7 & 74.3 & 20.1 & 5.5 & 25.7 & 45.8 & 29.6 & 2.3 & 77.7 & 21.9 & 0.4 & 22.3 & 54.8 & 28.2 & 1.8 & 84.8 & 15.2 & 0.0 & 15.2 \\
\hline & 20 & 33.1 & 23.9 & 2.0 & 59.1 & 32.2 & 8.7 & 40.9 & 51.5 & 30.7 & 1.2 & 83.4 & 16.3 & 0.3 & 16.6 & 59.0 & 34.0 & 1.1 & 94.0 & 6.0 & 0.0 & 6.0 \\
\hline \multirow{2}{*}{$x^{2 m}$} & 1 & 67.4 & 5.9 & 11.1 & 84.3 & 10.2 & 5.4 & 15.7 & 77.8 & 5.3 & 9.3 & 92.3 & 7.1 & 0.6 & 7.7 & 84.1 & 4.5 & 6.1 & 94.7 & 5.0 & 0.3 & 5.3 \\
\hline & 20 & 67.3 & 5.1 & 9.9 & 82.2 & 12.1 & 5.7 & 17.8 & 86.1 & 5.6 & 4.9 & 96.5 & 3.2 & 0.3 & 3.5 & 89.2 & 5.5 & 3.9 & 98.7 & 1.3 & 0.1 & 1.3 \\
\hline \multirow{2}{*}{$x^{3 m}$} & 1 & 73.3 & 1.3 & 12.1 & 86.7 & 5.4 & 7.9 & 13.3 & 82.3 & 1.1 & 9.1 & 92.6 & 3.3 & 4.1 & 7.4 & 88.0 & 0.9 & 6.0 & 94.9 & 2.4 & 2.7 & 5.1 \\
\hline & 20 & 74.8 & 1.1 & 11.0 & 86.9 & 5.3 & 7.8 & 13.1 & 91.1 & 1.2 & 5.0 & 97.3 & 1.2 & 1.5 & 2.7 & 93.8 & 1.1 & 4.0 & 99.0 & 0.5 & 0.6 & 1.0 \\
\hline \multirow{2}{*}{$x^{4 m}$} & 1 & 80.8 & 0.0 & 7.6 & 88.3 & 6.2 & 5.5 & 11.7 & 89.8 & 0.0 & 5.4 & 95.2 & 1.6 & 3.2 & 4.8 & 93.2 & 0.0 & 3.7 & 96.9 & 1.0 & 2.1 & 3.1 \\
\hline & 20 & 81.8 & 0.0 & 6.7 & 88.5 & 6.1 & 5.4 & 11.5 & 95.4 & 0.0 & 2.8 & 98.2 & 0.6 & 1.2 & 1.8 & 97.0 & 0.0 & 2.3 & 99.4 & 0.2 & 0.4 & 0.6 \\
\hline \multirow{2}{*}{$x^{5 m}$} & 1 & 84.1 & 0.7 & 3.1 & 87.9 & 6.1 & 6.0 & 12.1 & 93.5 & 0.7 & 2.4 & 96.6 & 1.0 & 2.4 & 3.4 & 95.7 & 0.5 & 1.6 & 97.8 & 0.6 & 1.6 & 2.2 \\
\hline & 20 & 80.6 & 0.6 & 2.6 & 83.8 & 8.3 & 7.9 & 16.2 & 96.5 & 0.6 & 1.2 & 98.3 & 0.5 & 1.2 & 1.7 & 97.8 & 0.6 & 1.0 & 99.4 & 0.2 & 0.4 & 0.6 \\
\hline \multirow{2}{*}{$x^{6 m}$} & 1 & 84.4 & 3.3 & 0.7 & 88.4 & 7.2 & 4.3 & 11.6 & 93.3 & 2.8 & 0.6 & 96.7 & 1.4 & 1.9 & 3.3 & 95.3 & 2.3 & 0.4 & 97.9 & 0.7 & 1.3 & 2.1 \\
\hline & 20 & 79.2 & 2.7 & 0.5 & 82.4 & 11.2 & 6.4 & 17.6 & 95.1 & 2.7 & 0.3 & 98.1 & 0.8 & 1.1 & 1.9 & 96.4 & 2.7 & 0.2 & 99.3 & 0.2 & 0.4 & 0.7 \\
\hline \multirow{2}{*}{$x^{7 m}$} & 1 & 83.5 & 4.5 & 0.0 & 87.9 & 9.3 & 2.8 & 12.1 & 94.7 & 3.8 & 0.0 & 98.5 & 0.6 & 0.9 & 1.5 & 95.7 & 3.1 & 0.0 & 98.8 & 0.4 & 0.8 & 1.2 \\
\hline & 20 & 80.0 & 3.7 & 0.0 & 83.7 & 12.7 & 3.6 & 16.3 & 95.7 & 3.6 & 0.0 & 99.3 & 0.3 & 0.4 & 0.7 & 96.1 & 3.5 & 0.0 & 99.7 & 0.1 & 0.2 & 0.3 \\
\hline \multirow{2}{*}{$x^{8 m}$} & 1 & 85.4 & 5.6 & 0.6 & 91.6 & 6.2 & 2.2 & 8.4 & 93.5 & 4.6 & 0.4 & 98.6 & 0.9 & 0.6 & 1.4 & 95.1 & 3.7 & 0.3 & 99.1 & 0.5 & 0.4 & 0.9 \\
\hline & 20 & 86.2 & 4.9 & 0.5 & 91.5 & 6.2 & 2.2 & 8.5 & 94.8 & 4.5 & 0.2 & 99.5 & 0.3 & 0.2 & 0.5 & 95.3 & 4.3 & 0.2 & 99.8 & 0.1 & 0.1 & 0.2 \\
\hline \multirow{2}{*}{$x^{9 m}$} & 1 & 82.4 & 6.7 & 2.4 & 91.5 & 7.6 & 0.9 & 8.5 & 91.0 & 5.6 & 1.7 & 98.4 & 0.9 & 0.7 & 1.6 & 93.3 & 4.6 & 1.1 & 98.9 & 0.6 & 0.4 & 1.1 \\
\hline & 20 & 84.9 & 6.0 & 2.2 & 93.1 & 6.2 & 0.7 & 6.9 & 93.2 & 5.5 & 0.9 & 99.5 & 0.3 & 0.2 & 0.5 & 93.8 & 5.4 & 0.7 & 99.8 & 0.1 & 0.1 & 0.2 \\
\hline \multirow{2}{*}{$x^{10 m}$} & 1 & 77.9 & 7.3 & 4.6 & 89.7 & 8.2 & 2.1 & 10.3 & 88.1 & 6.4 & 3.2 & 97.6 & 0.6 & 1.8 & 2.4 & 91.2 & 5.1 & 2.2 & 98.5 & 0.5 & 1.0 & 1.5 \\
\hline & 20 & 80.9 & 6.6 & 4.1 & 91.6 & 6.6 & 1.7 & 8.4 & 91.4 & 6.3 & 1.7 & 99.3 & 0.2 & 0.5 & 0.7 & 92.3 & 6.1 & 1.4 & 99.8 & 0.1 & 0.2 & 0.2 \\
\hline & 1 & 73.4 & 7.8 & 6.9 & 88.1 & 5.5 & 6.3 & 11.9 & 84.7 & 7.0 & 5.1 & 96.8 & 0.9 & 2.3 & 3.2 & 88.4 & 5.7 & 3.6 & 97.7 & 0.7 & 1.7 & 2.3 \\
\hline$x^{1+m}$ & 20 & 74.2 & 6.9 & 6.1 & 87.1 & 6.1 & 6.8 & 12.9 & 89.1 & 7.0 & 2.7 & 98.7 & 0.4 & 0.9 & 1.3 & 90.5 & 6.8 & 2.2 & 99.5 & 0.2 & 0.4 & 0.5 \\
\hline & 1 & 64.9 & 8.0 & 9.0 & 81.9 & 7.9 & 10.1 & 18.1 & 80.0 & 7.7 & 7.4 & 95.0 & 0.6 & 4.4 & 5.0 & 84.8 & 6.3 & 5.2 & 96.3 & 0.7 & 2.9 & 3.7 \\
\hline & 20 & 62.6 & 6.7 & 7.5 & 76.8 & 10.4 & 12.8 & 23.2 & 85.9 & 7.8 & 3.8 & 97.5 & 0.3 & 2.2 & 2.5 & 88.1 & 7.7 & 3.2 & 99.0 & 0.2 & 0.8 & 1.0 \\
\hline
\end{tabular}

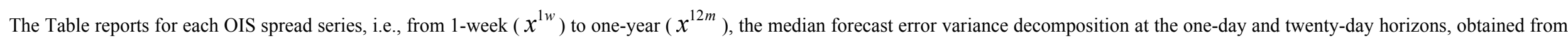

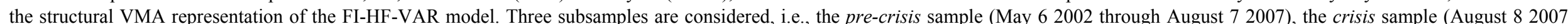

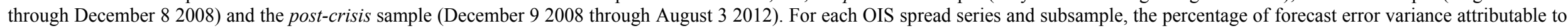

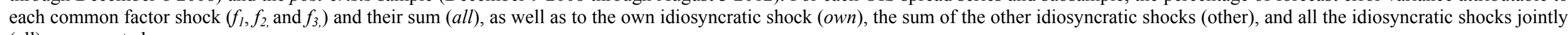
(all), are reported. 

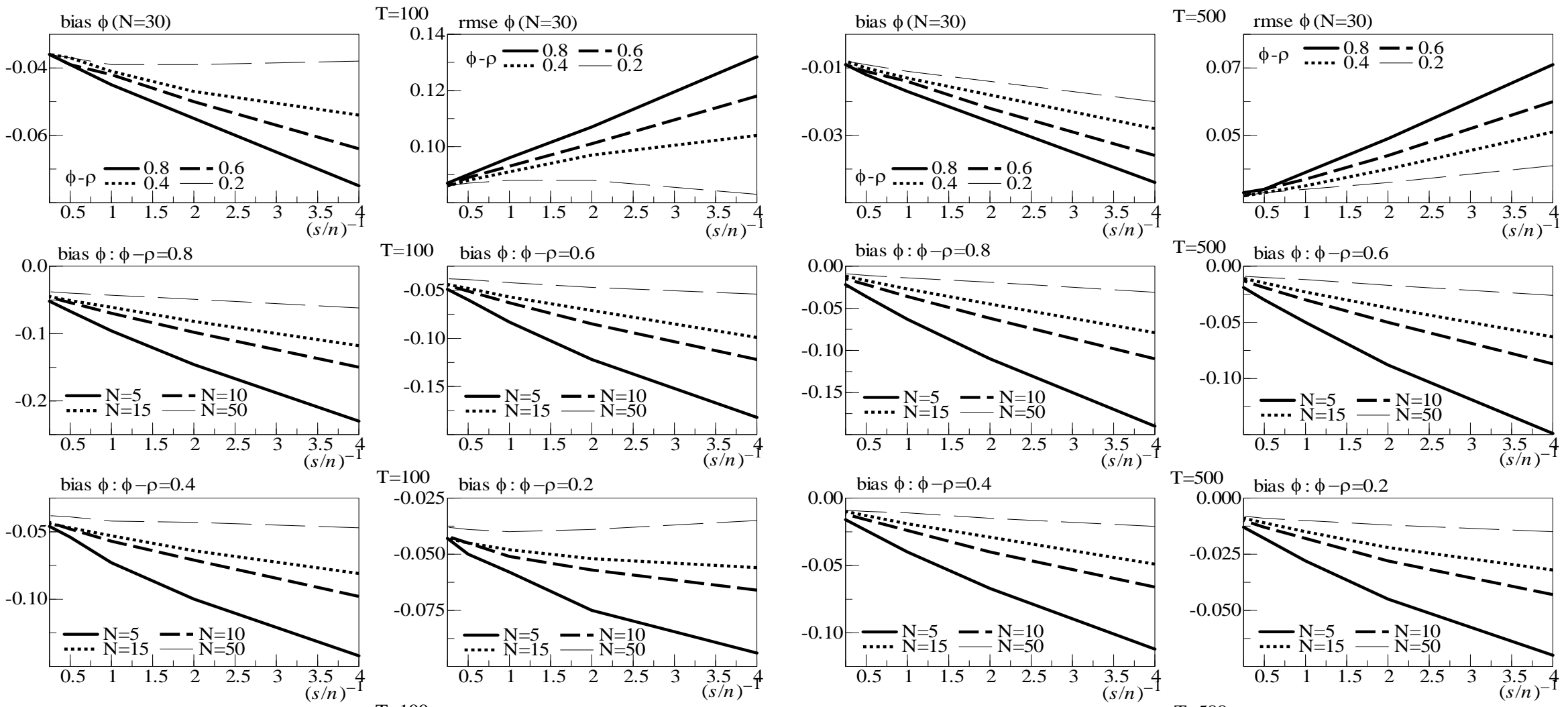

$\mathrm{T}=500 \quad$ bias $\phi: \phi-\rho=0.2$
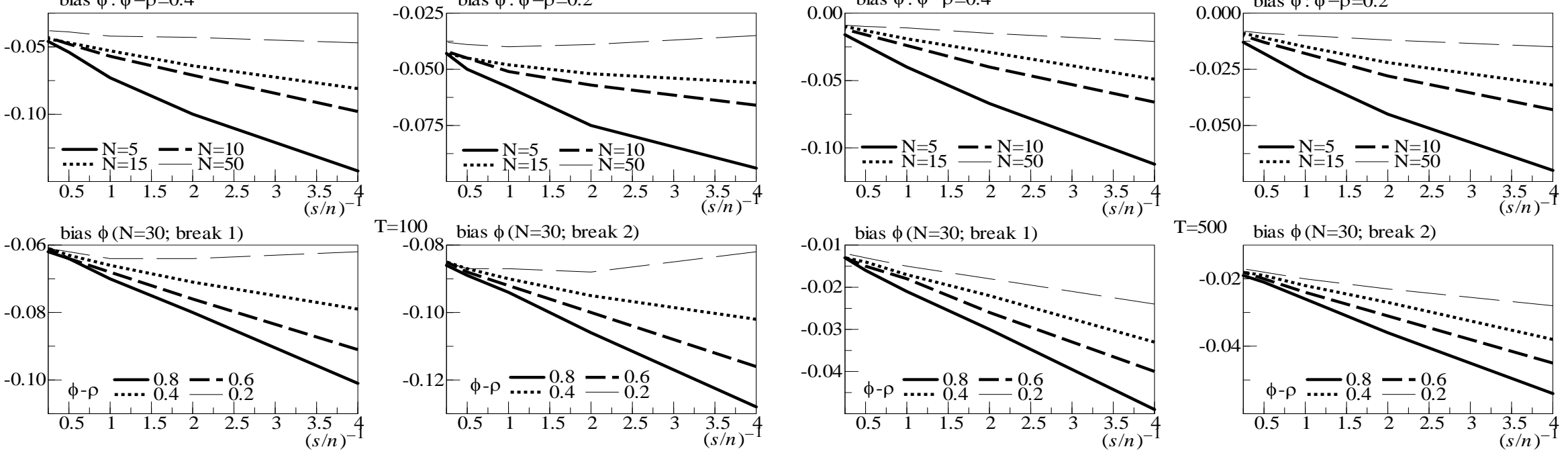

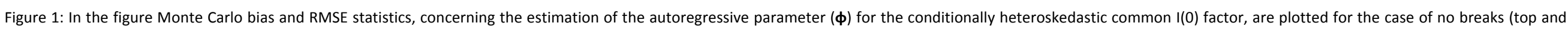

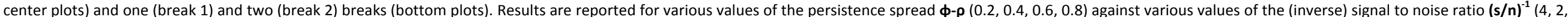

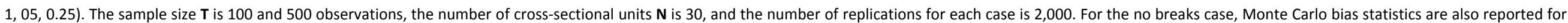
other sample sizes $N(5,10,15,50)$ (center plots). 

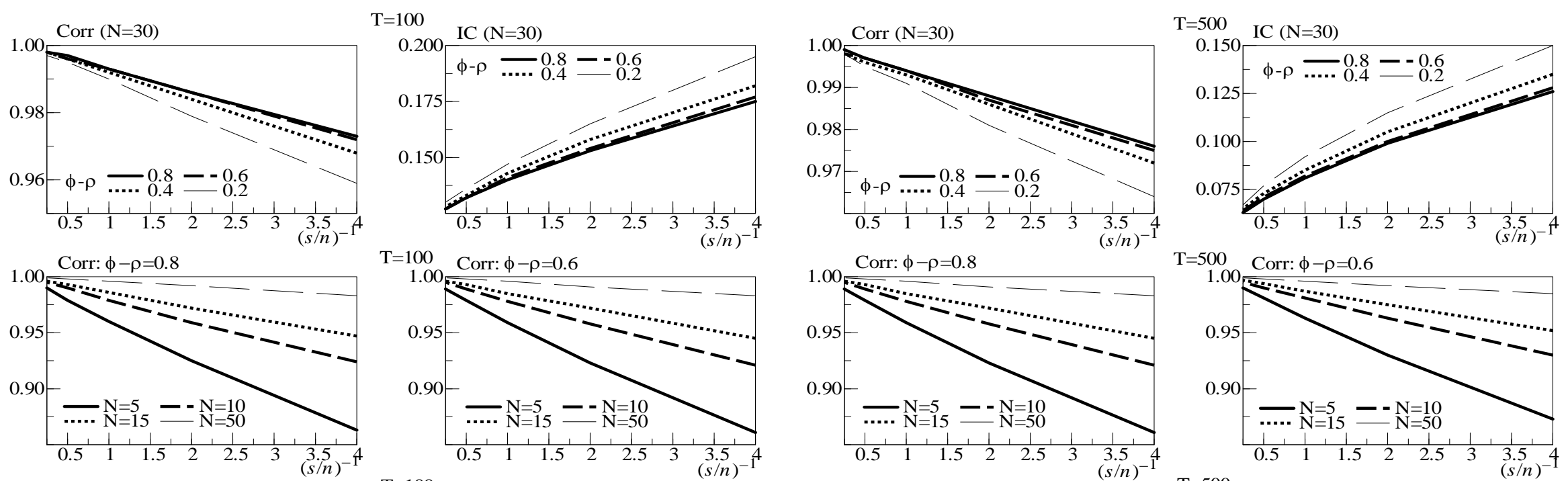

$\mathrm{T}=500$ Corr: $\phi-\rho=0.6$
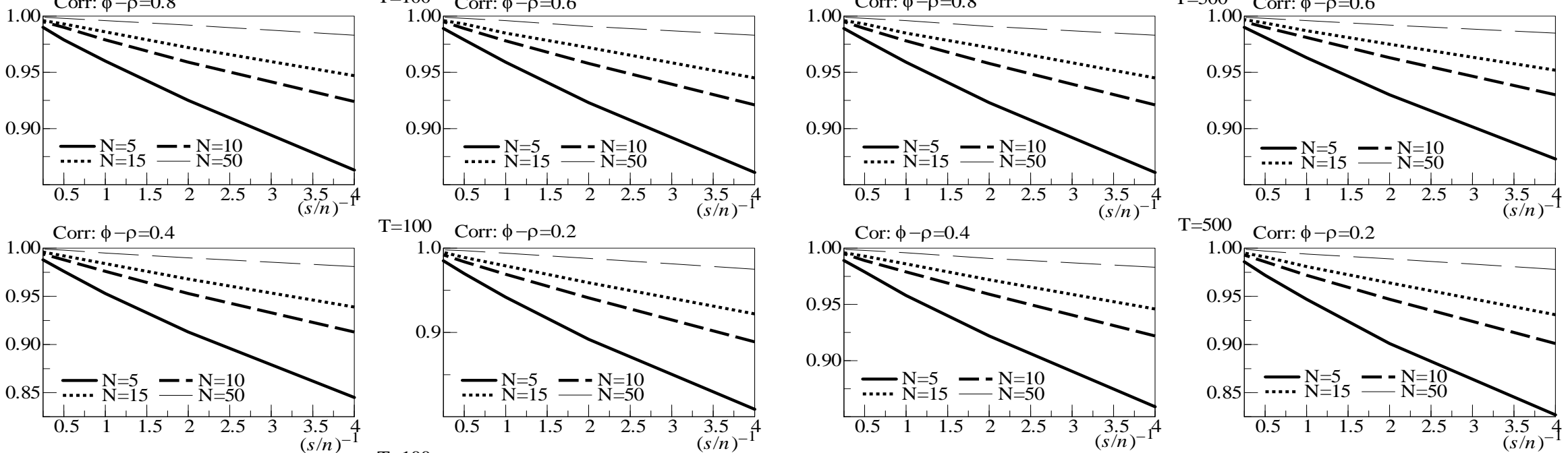

$\mathrm{T}=500$ Corr: $\phi-\rho=0.2$
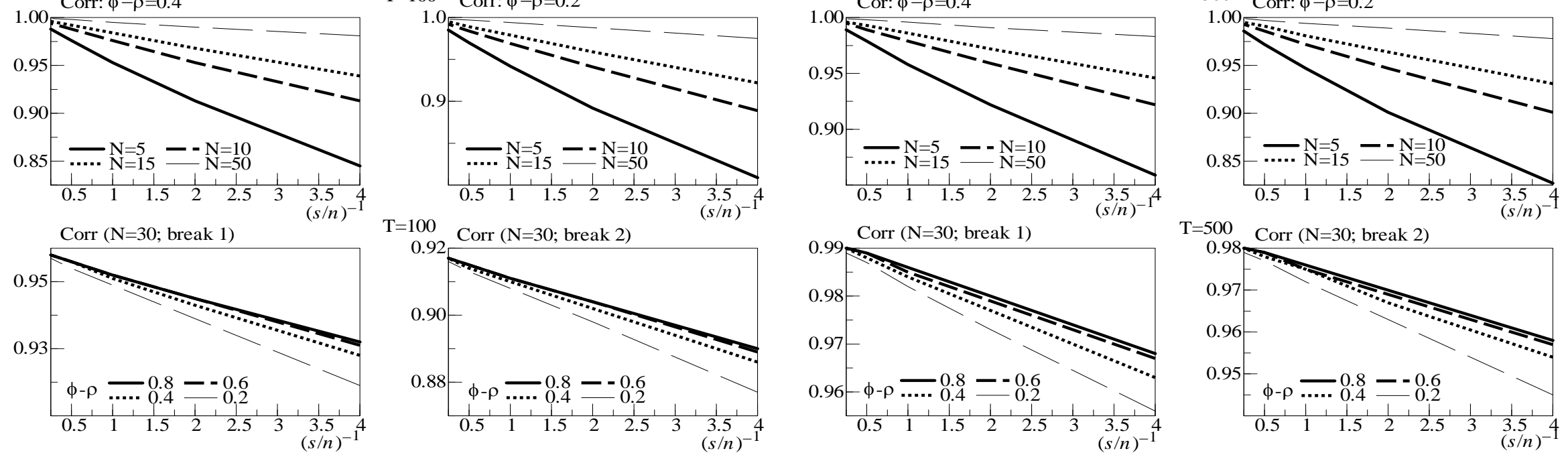

$\mathrm{T}=500$ Corr $(\mathrm{N}=30$; break 2$)$

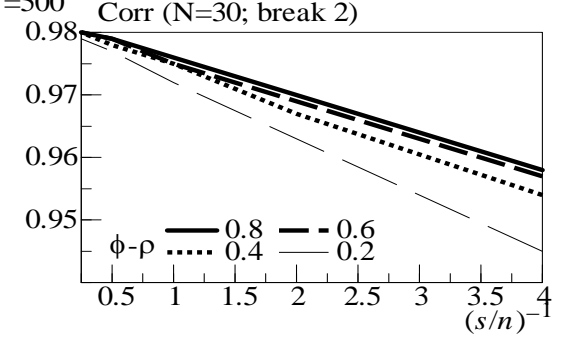

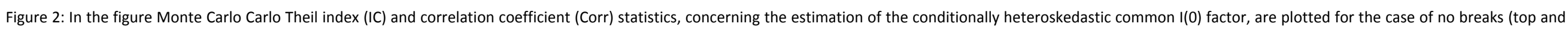

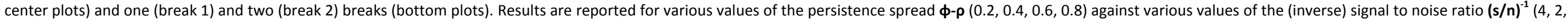

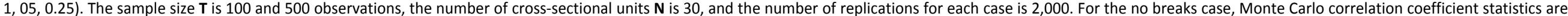
also reported for other sample sizes $\mathrm{N}(5,10,15,50)$ (center plots). 


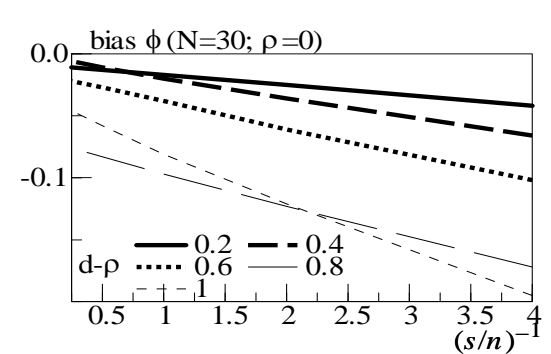

$\mathrm{T}=100$
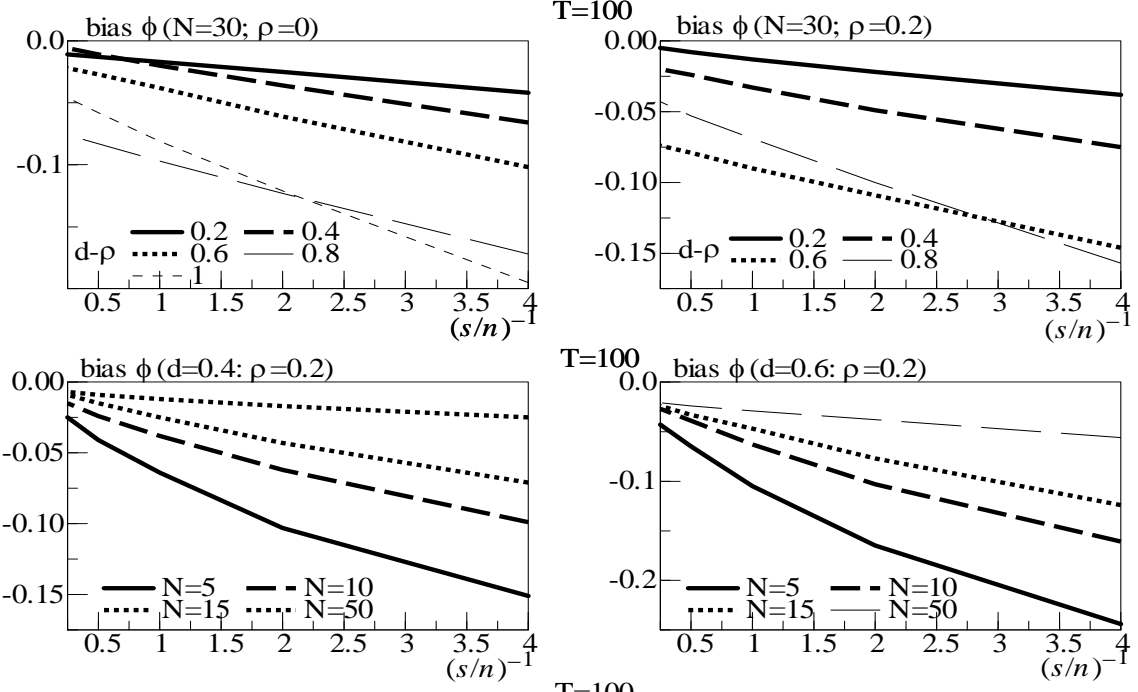

$\mathrm{T}=100$ bias $\phi(d=0.6: \rho=0.2)$

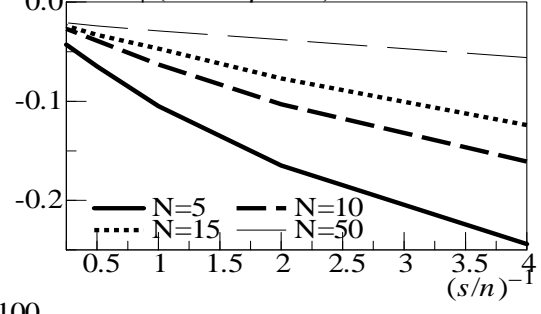

$\mathrm{T}=100$

0.00 bias $\phi(N=30 ; \rho=0 ;$ break 1$)$

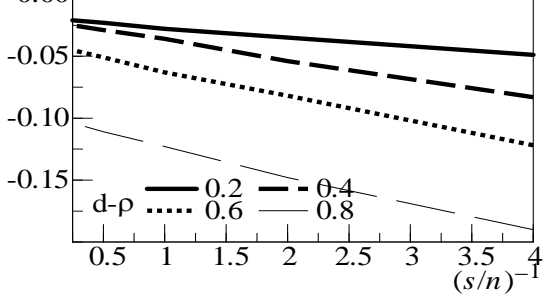

0.0 bias $\phi(N=30 ; \rho=0$; break 2) $(s / n)^{-1}$

0.00 bias $\phi(N=30 ; \rho=0.2$; break 1$)$

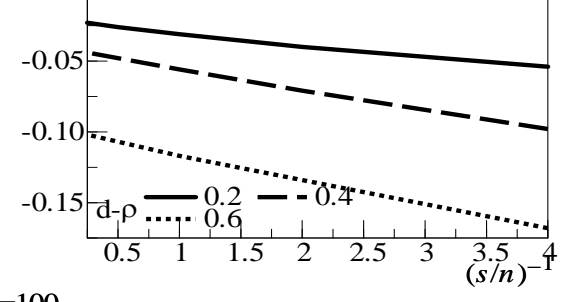

$\mathrm{T}=100$

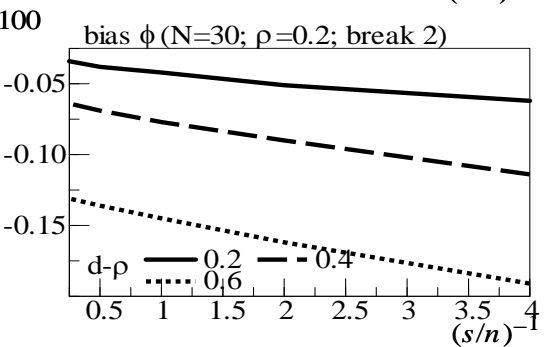

bias $\phi(N=30 ; \rho=0)$

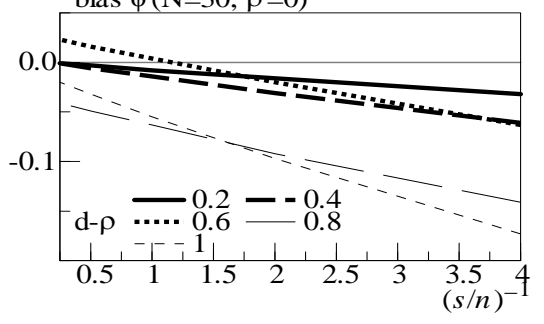

bias $\phi(d=0.4: \rho=0.2)$

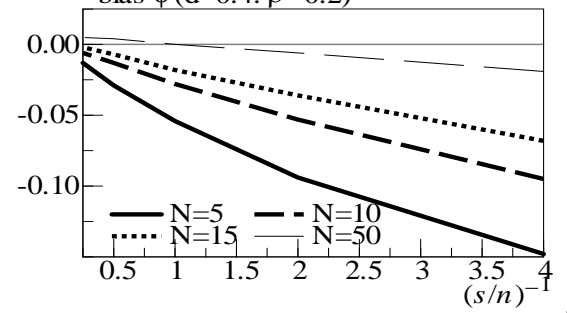

bias $\phi(N=30 ; \rho=0$; break 1$)$

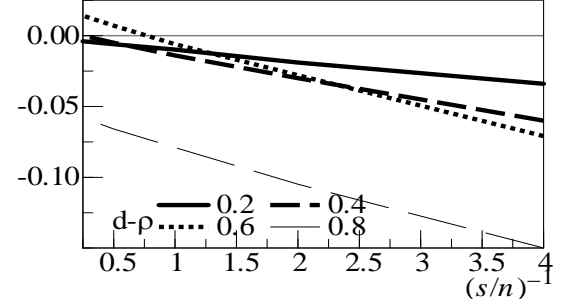

0.0 bias $\phi(\mathrm{N}=30 ; \rho=0$; break 2$)$

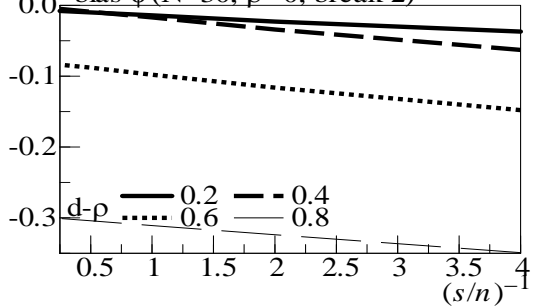

$\mathrm{T}=500$ bias $\phi(\mathrm{N}=30 ; \rho=0.2)$

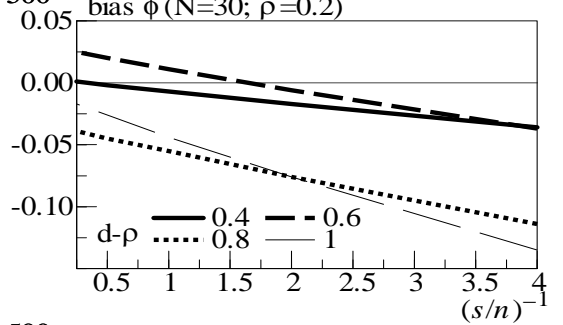

$\mathrm{T}=500 \quad$ bias $\phi(d=0.6: \rho=0.2)$

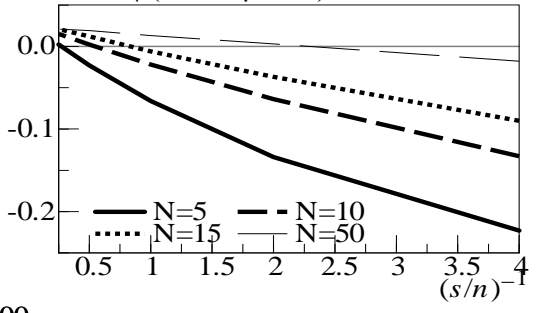

$\mathrm{T}=500$

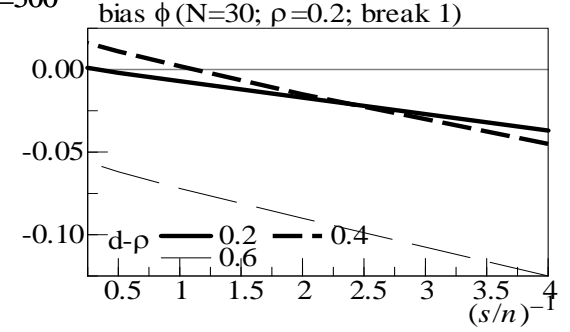

$\mathrm{T}=500 \quad$ bias $\phi(N=30 ; \rho=0.2$; break 2$)$

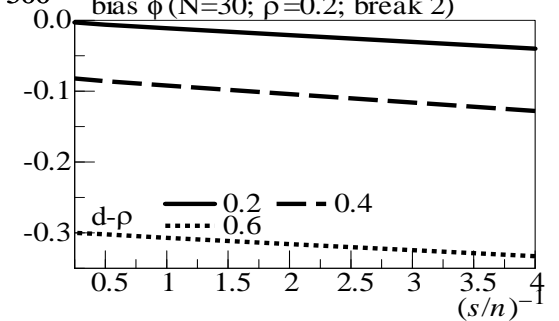

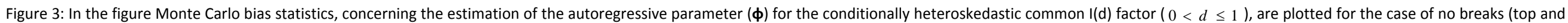

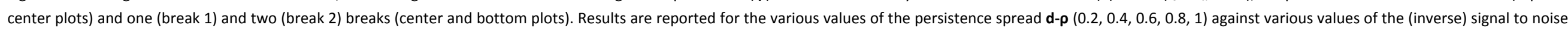

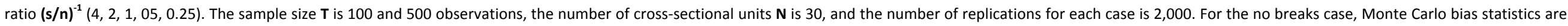
also reported for other sample sizes $\mathrm{N}(5,10,15,50)$ (center plots). 

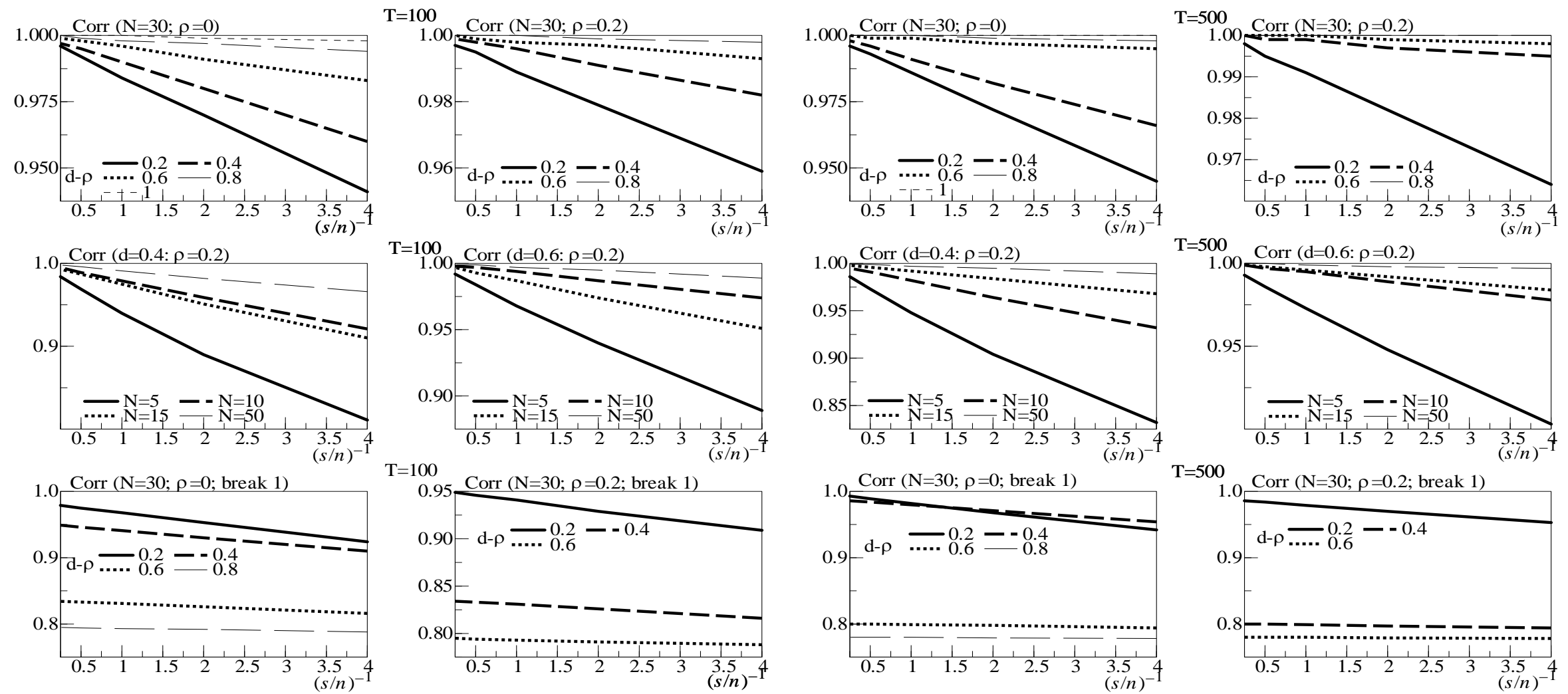

1.0 $=500$ Corr $(\mathrm{N}=30 ; \rho=0.2$; break 1$)$
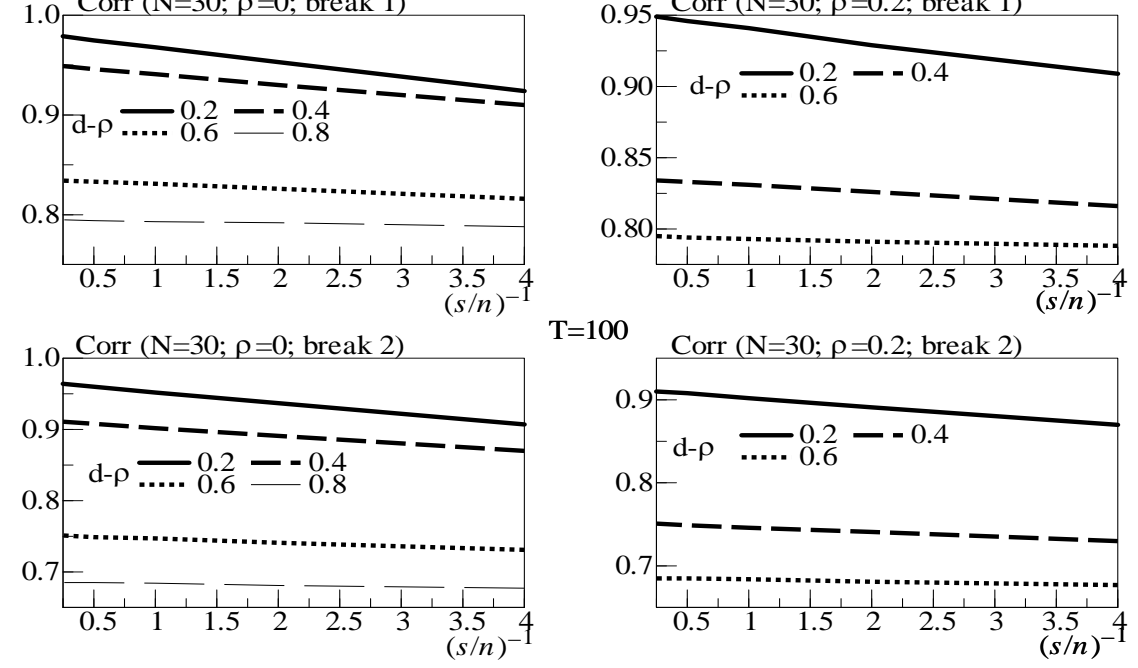

$\mathrm{T}=100 \quad \operatorname{Corr}(\mathrm{N}=30 ; \rho=0.2$; break 2$)$
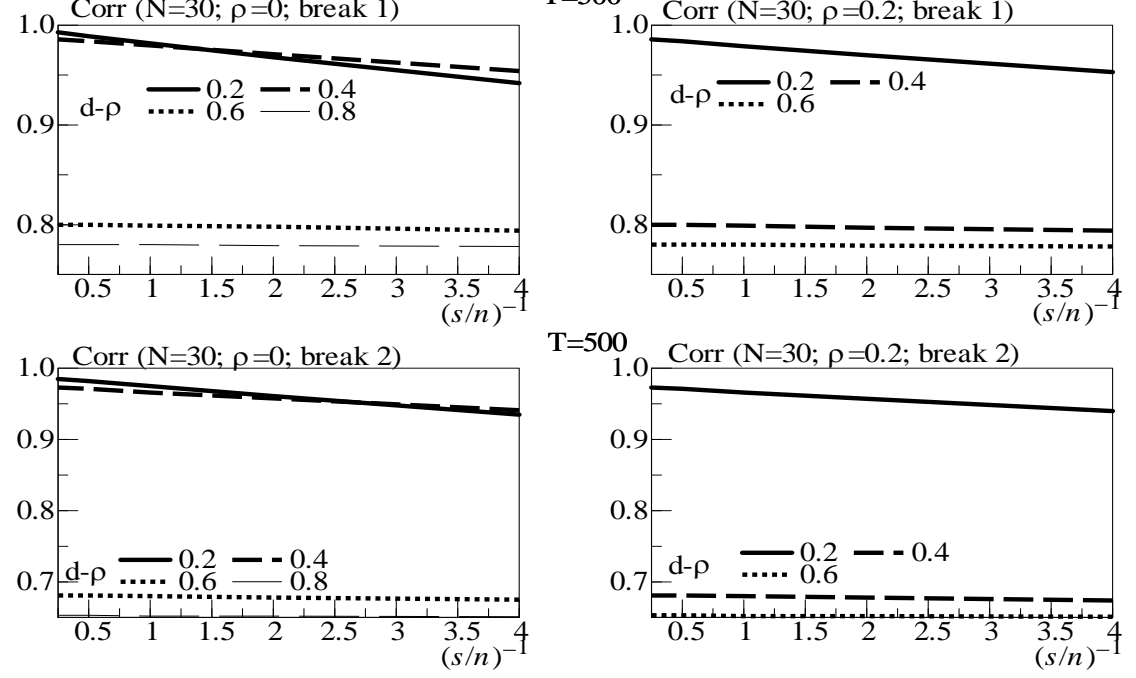

$\mathrm{T}=500$

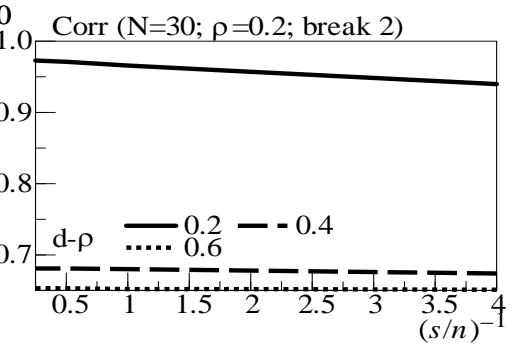

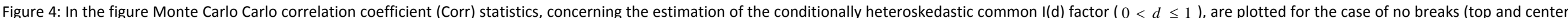

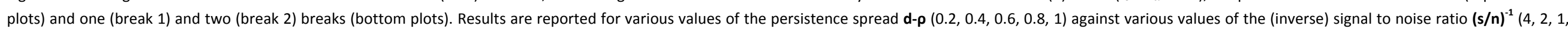

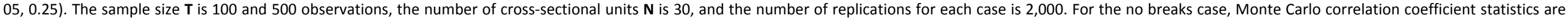
also reported for other sample sizes $\mathrm{N}(5,10,15,50)$ (center plots). 



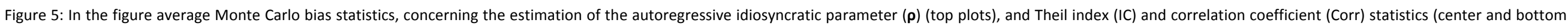

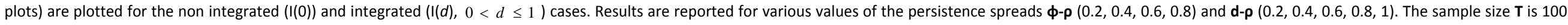
and 500 observations, the number of cross-sectional units $\mathbf{N}$ is 30 , and the number of replications for each case is 2,000. 

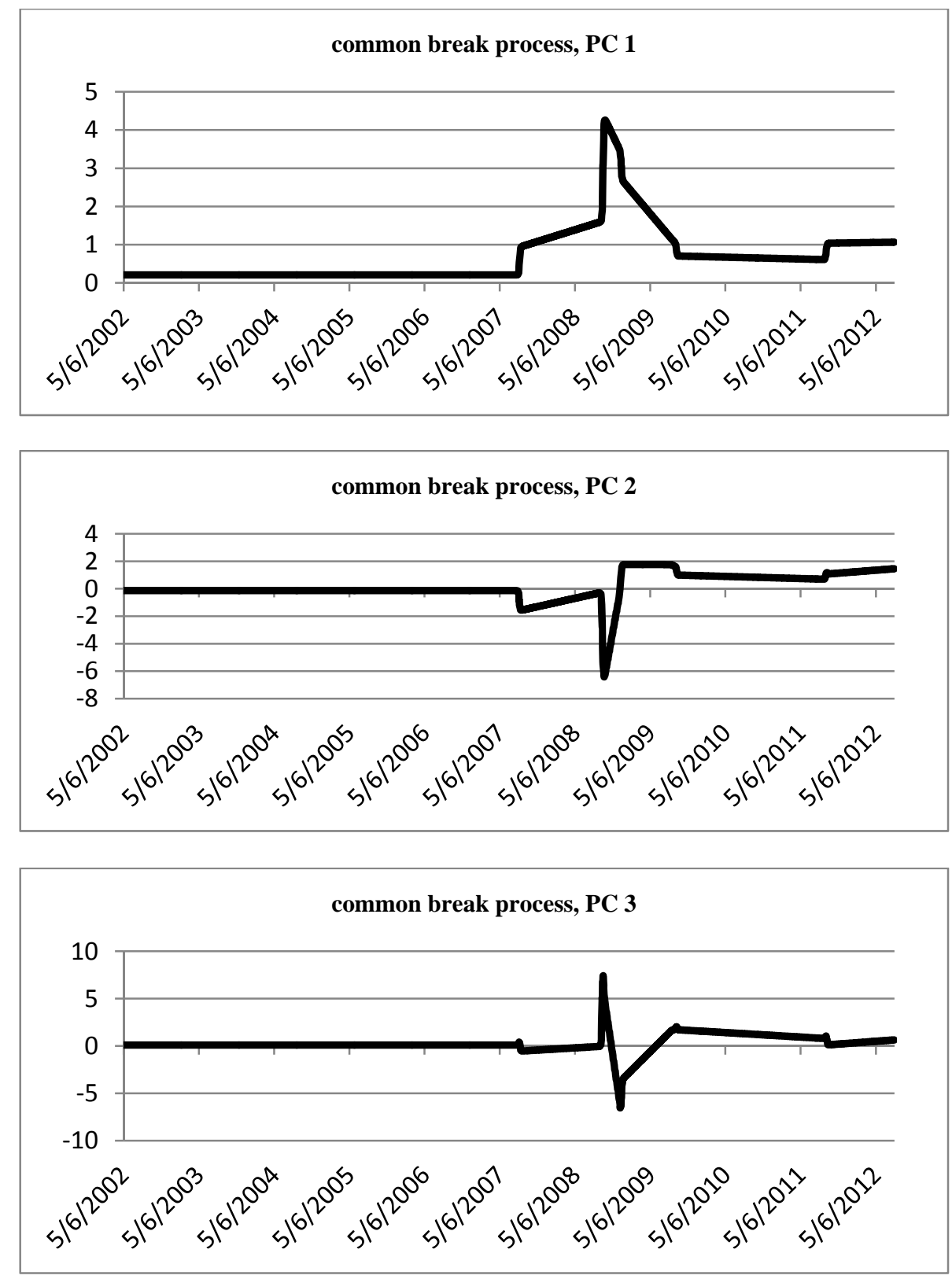

Figure 6: common estimated components from OIS spreads break process and normalized break-free series.
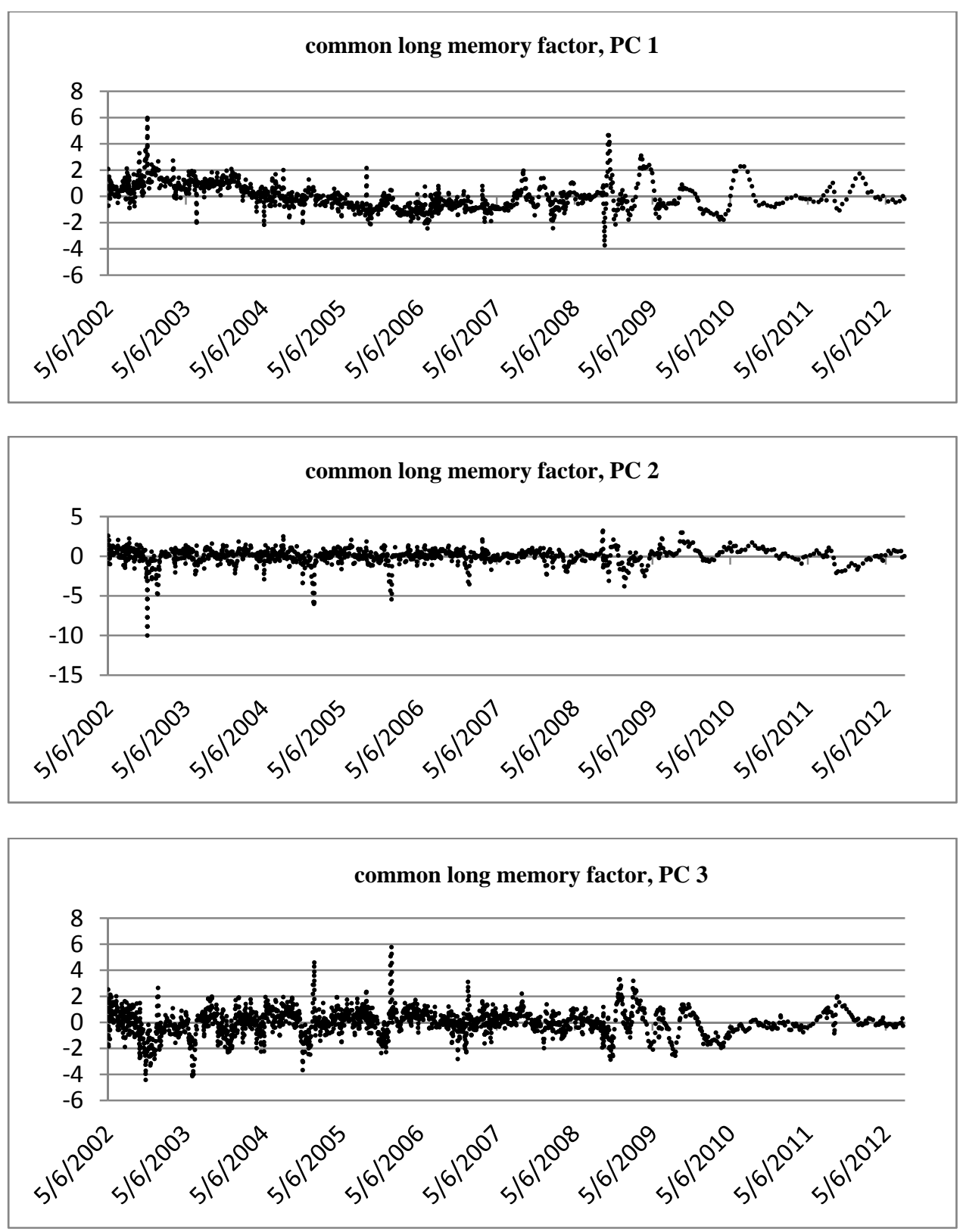

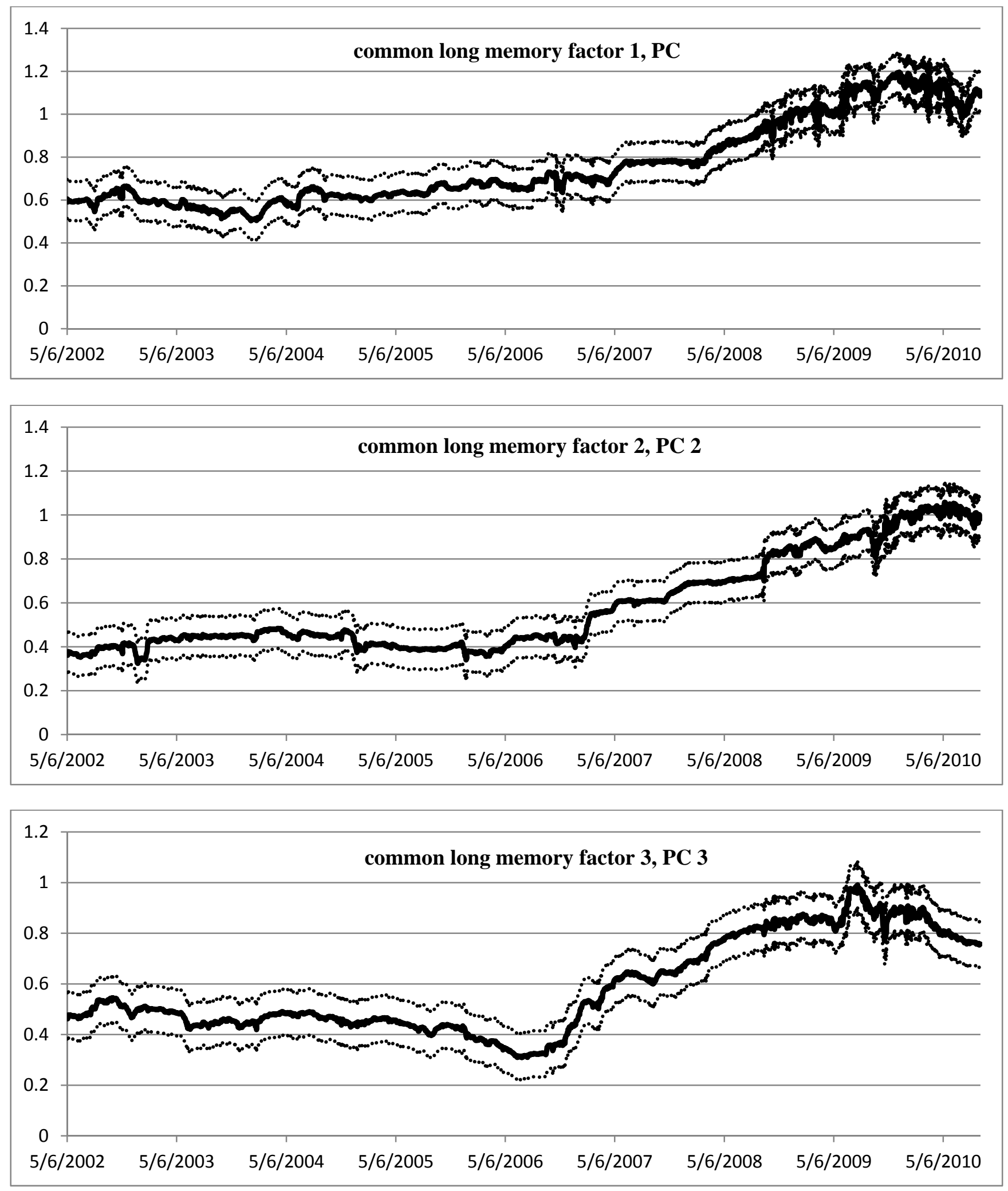

Figure 7: Moulines and Soulier (1999) broad band log periodogram moving window estimates of the fractional differencing parameter, plus and minus one standard error; estimates are for the first three principal components (PC) extracted from the OIS spreads normalized break-free series. 


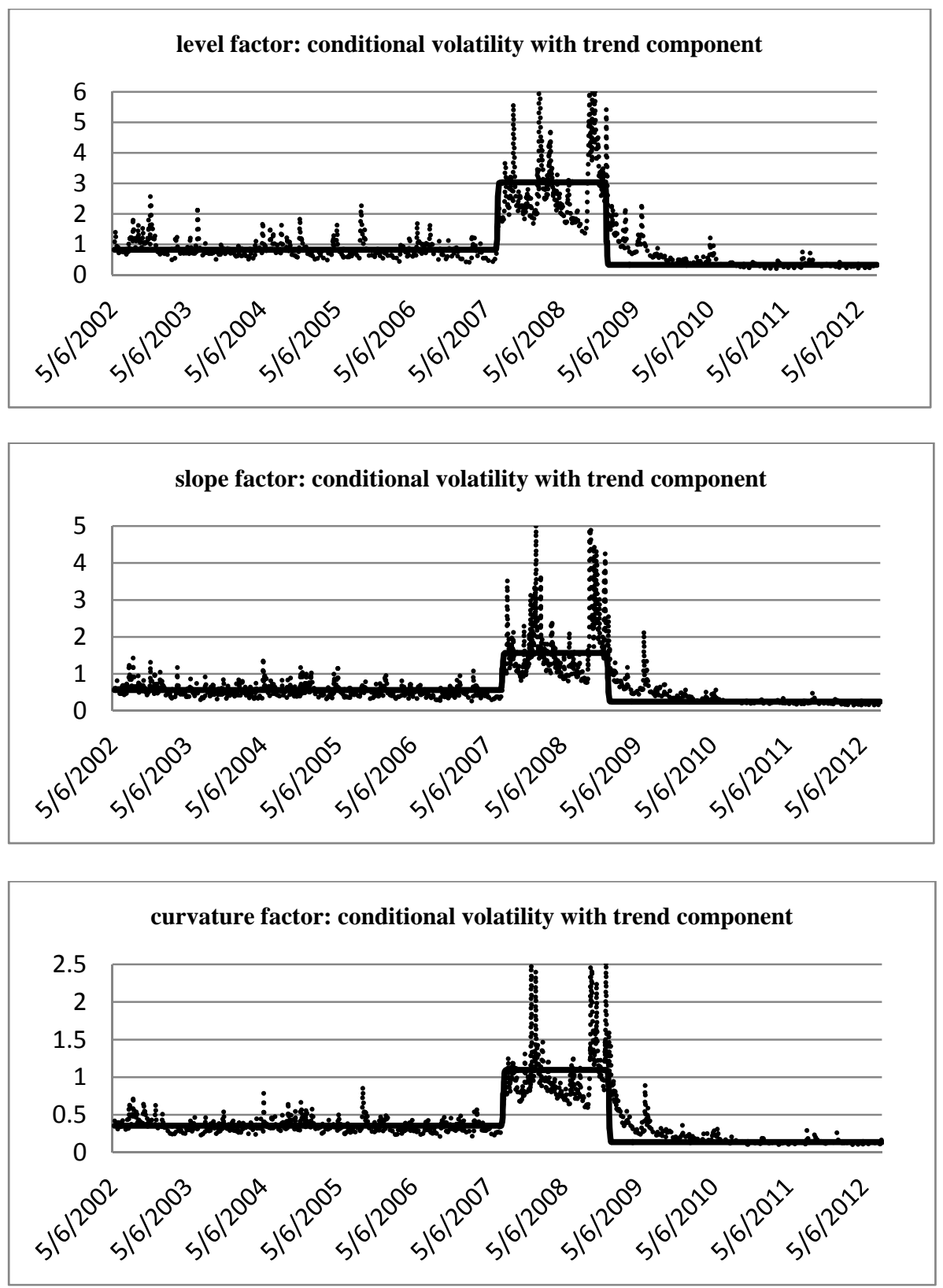

Figure 8: FI-H-FVAR model estimates of common factors in mean and variance. 

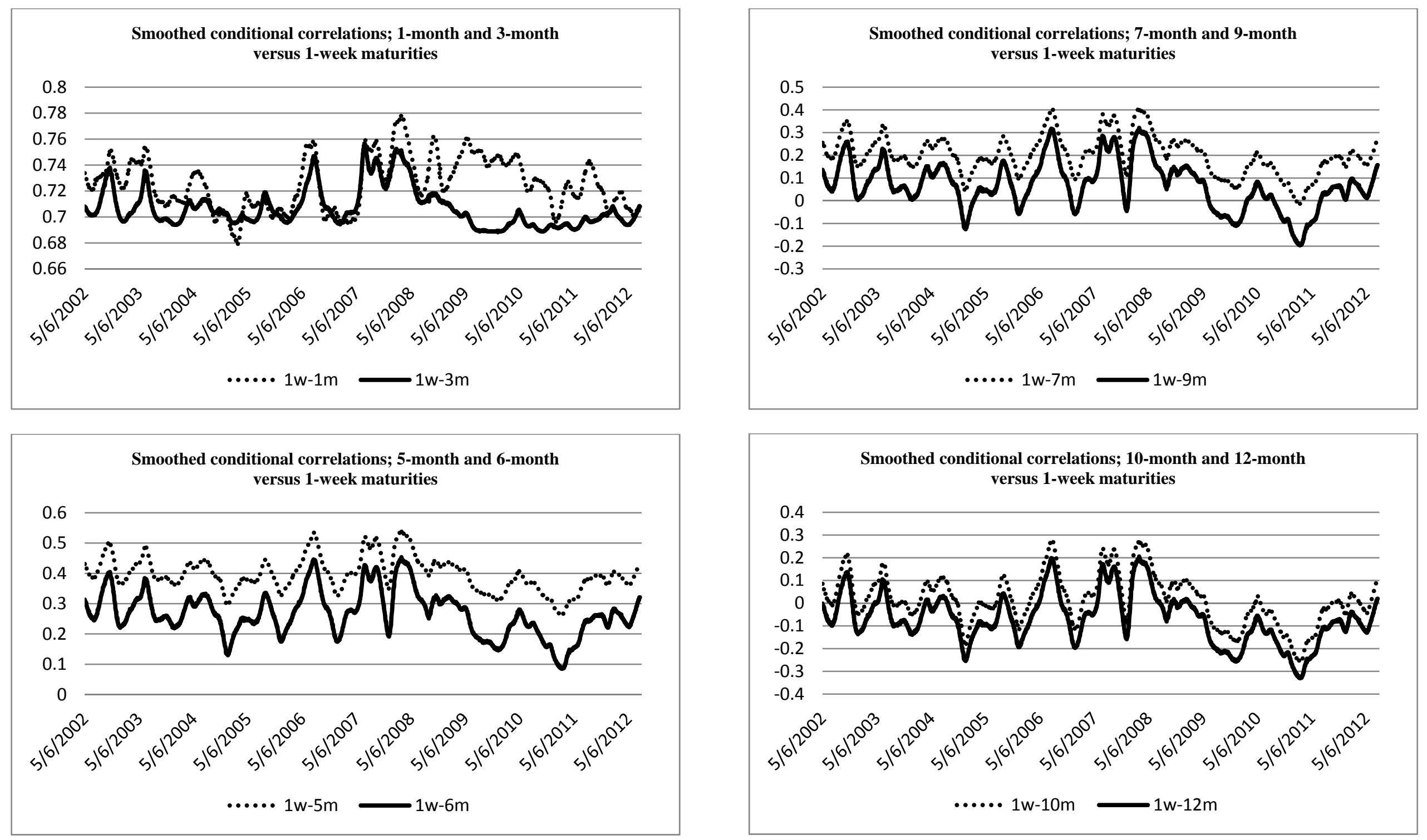

Figure 9: FI-H-FVAR model estimates of conditional correlations; various OIS spreads montly maturities (m) versus the 1-week OIS spread maturity (1w). 

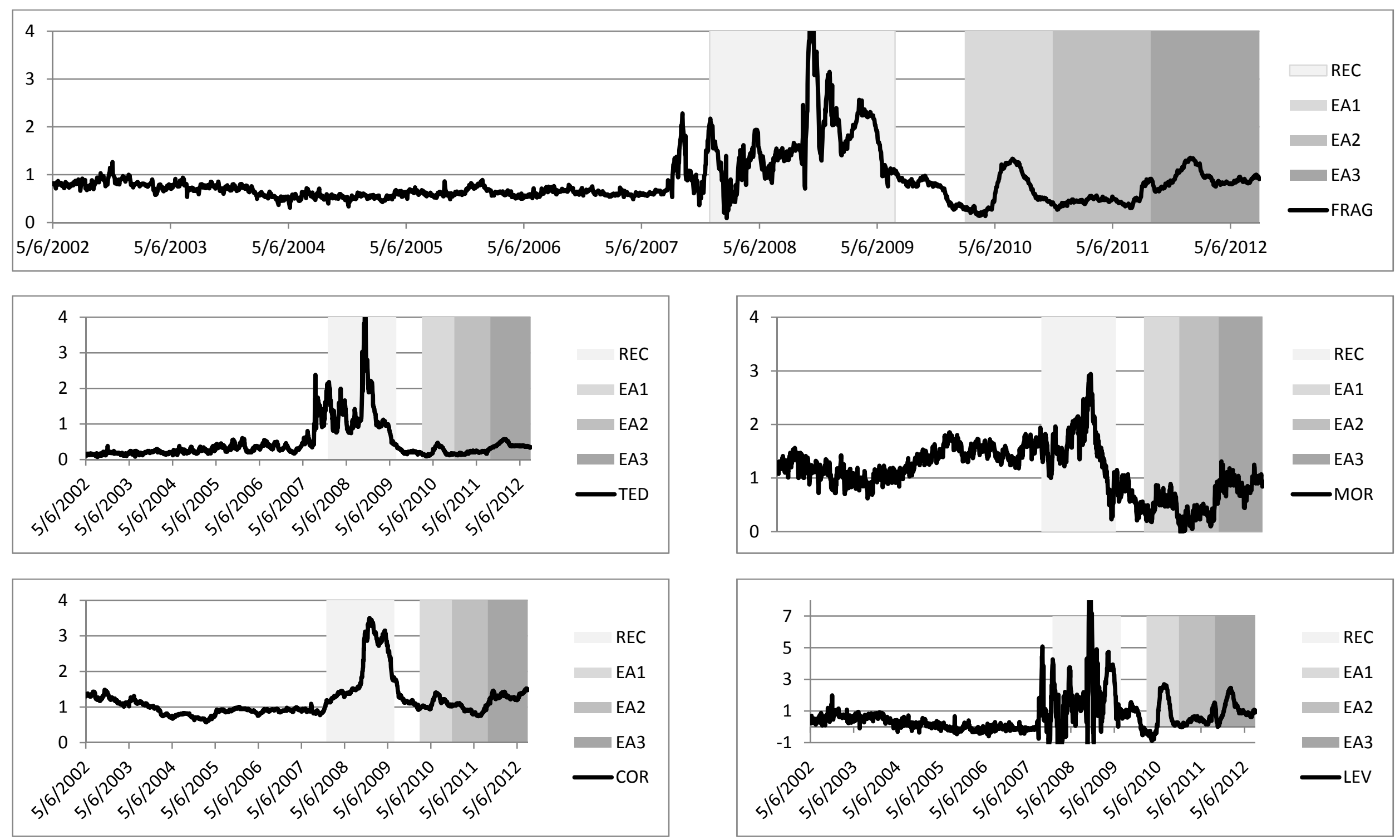

Figure 10: Risk measures: composite fragility indicator (FRAG), TED spread (TED), corporate spread (COR), OIS spreads level factor (LEV), mortgage spread (MOR); shaded areas refer to the December 2007 through June 2009 US recession (REC) and the three phases of the euro area sovereign debt crisis, i.e. Febraury 2010 through October 2010 (EA1), November 2010 through August 2011 (EA2) and September 2011 through July 2012 (EA3). 

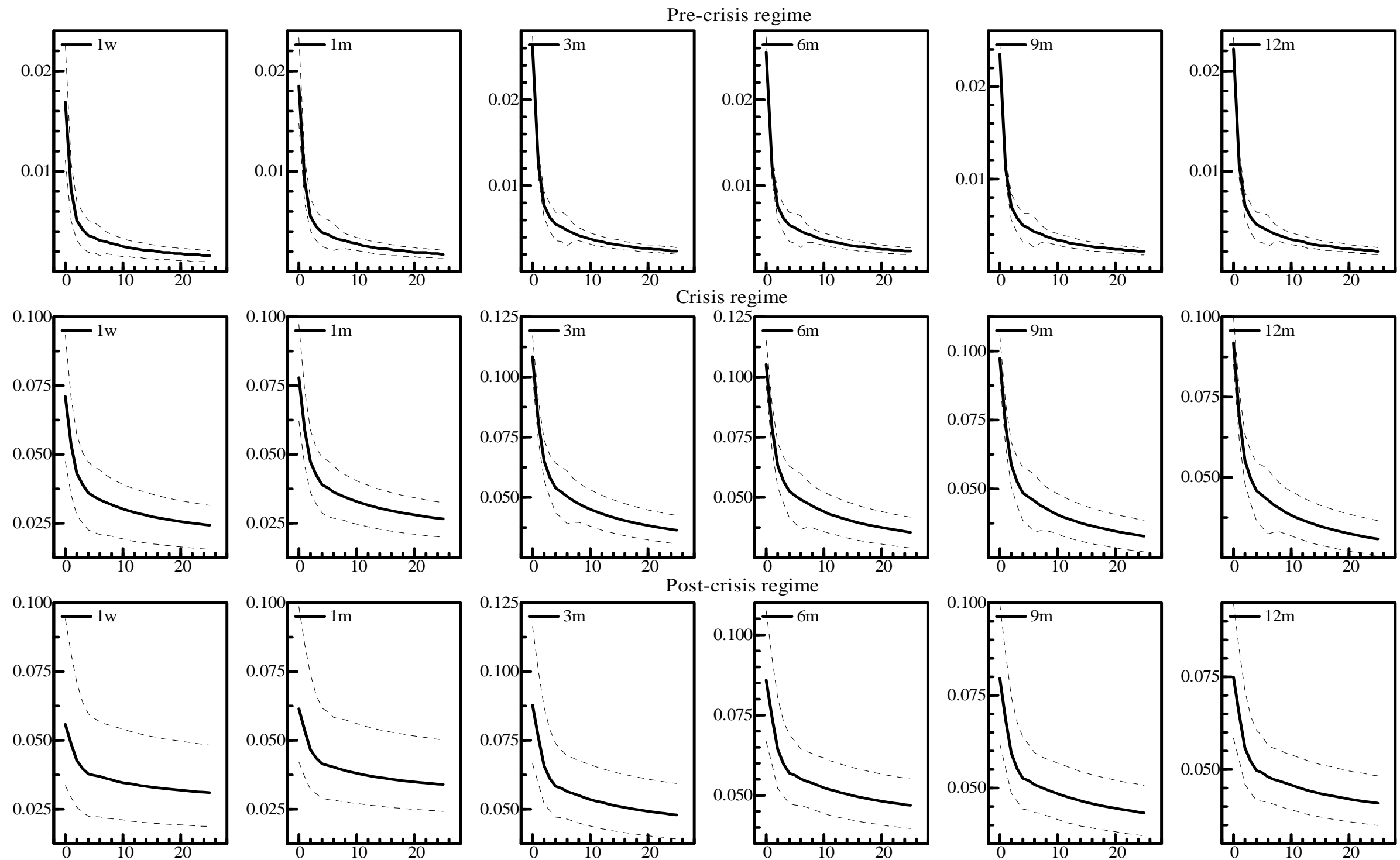

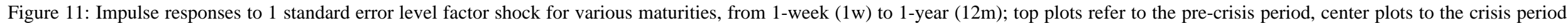
and bottom plots to the post-crisis period. 

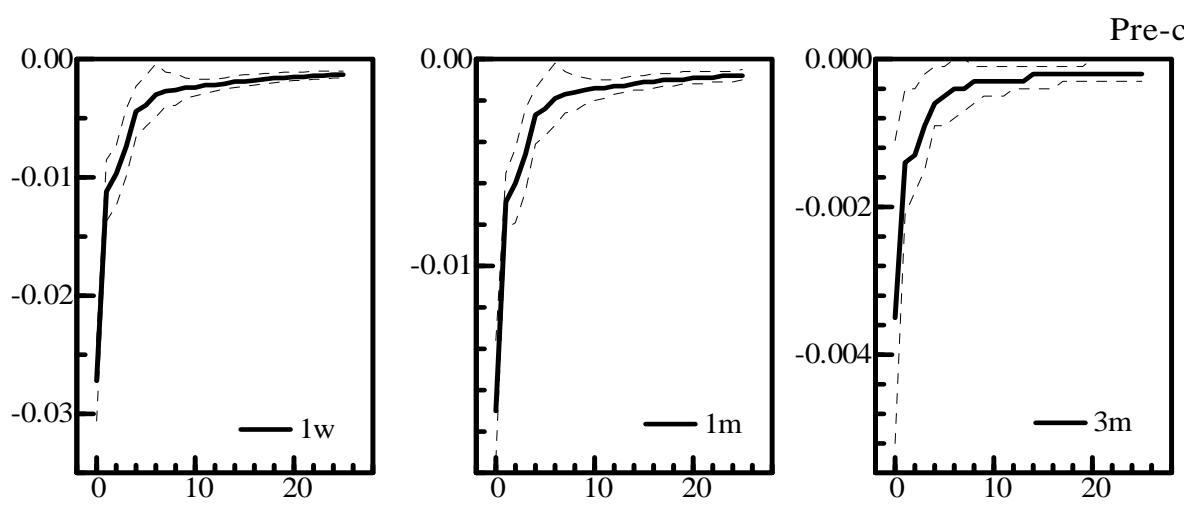

Pre-crisis regime
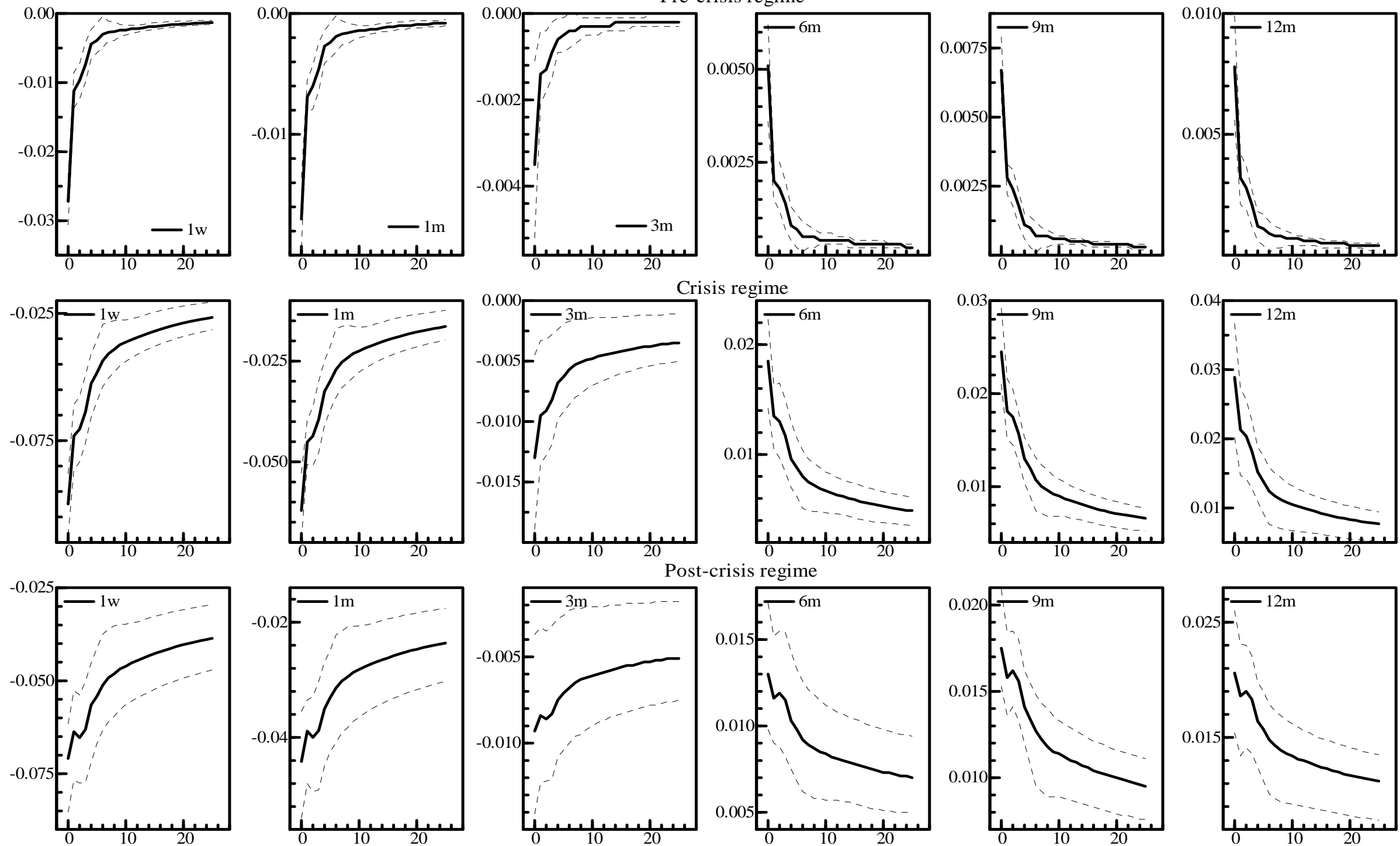

Figure 12: Impulse responses to 1 standard error slope factor shock for various maturities, from 1-week (1w) to 1-year (12m); top plots refer to the pre-crisis period, center plots to the crisis period and bottom plots to the post-crisis period. 

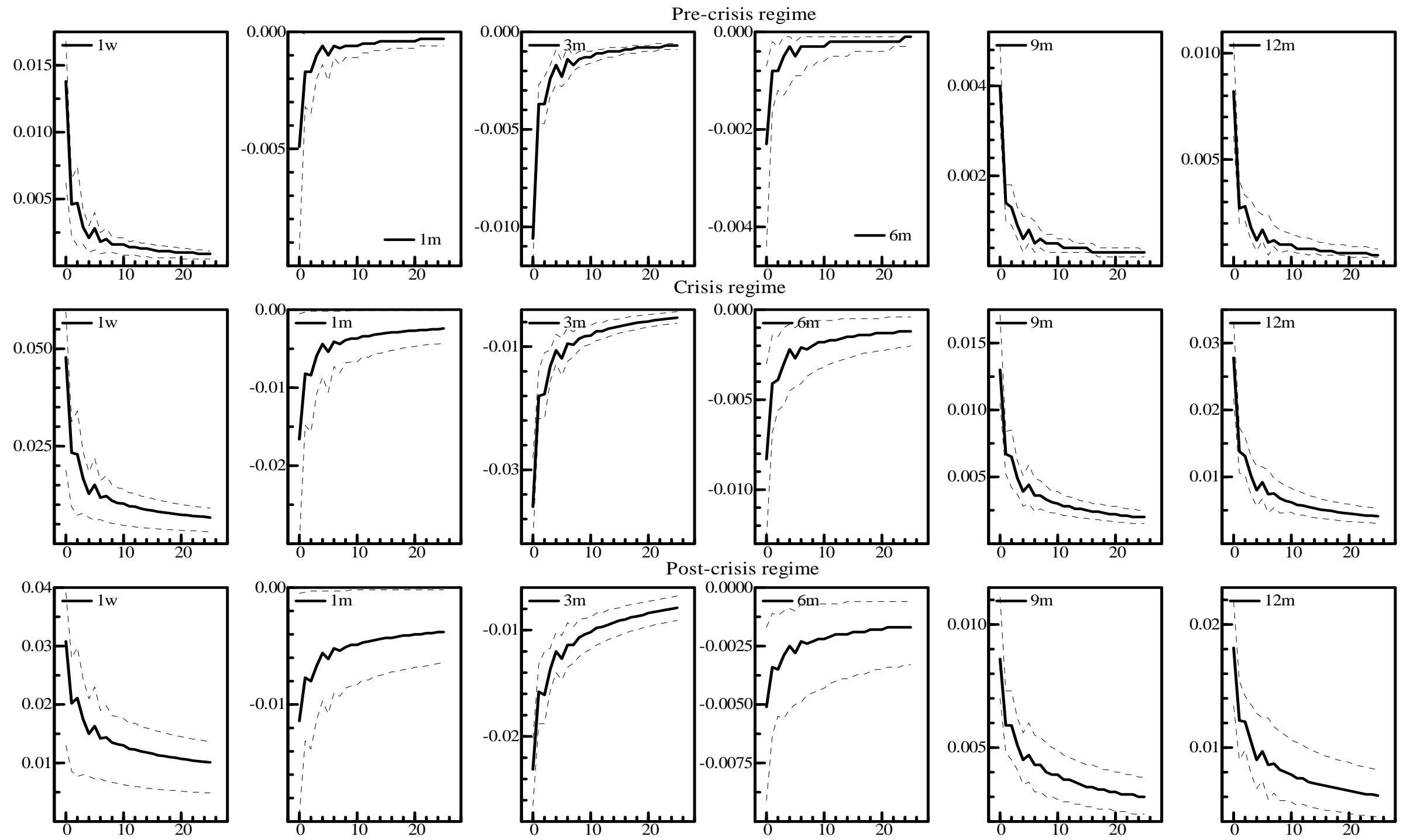

Figure 13: Impulse responses to 1 standard error curvature factor shock for various maturities, from 1-week (1w) to 1-year (12m); top plots refer to the pre-crisis period, center plots to the crisis period and bottom plots to the post-crisis period. 